\title{
Higgs-electroweak chiral Lagrangian: One-loop renormalization group equations
}

\author{
G. Buchalla $\odot,{ }^{1}$ O. Catà $\odot,{ }^{2}$ A. Celis $\odot,{ }^{1}$ M. Knecht $\odot,{ }^{3}$ and C. Krause $\odot^{4,5}$ \\ ${ }^{1}$ Ludwig-Maximilians-Universität München, Fakultät für Physik, Arnold Sommerfeld Center \\ for Theoretical Physics, D-80333 München, Germany \\ ${ }^{2}$ Theoretische Physik 1, Universität Siegen, Walter-Flex-Straße 3, D-57068 Siegen, Germany \\ ${ }^{3}$ Centre de Physique Théorique, CNRS/Aix-Marseille Univ./Univ. du Sud Toulon-Var (UMR 7332) \\ CNRS-Luminy Case 907, 13288 Marseille Cedex 9, France \\ ${ }^{4}$ Theoretical Physics Department, Fermi National Accelerator Laboratory, Batavia, Illinois 60510, USA \\ ${ }^{5}$ NHETC, Department of Physics and Astronomy, Rutgers University, Piscataway, New Jersey 08854, USA
}

(Received 12 May 2020; accepted 1 August 2021; published 6 October 2021)

\begin{abstract}
Starting from the one-loop divergences we obtained previously, we work out the renormalization of the Higgs-electroweak chiral Lagrangian explicitly and in detail. This includes the renormalization of the lowest-order Lagrangian, as well as the decomposition of the remaining divergences into a complete basis of next-to-leading-order counterterms. We provide the list of the corresponding beta functions. We show how our results match the one-loop renormalization of some of the dimension-6 operators in SMEFT. We further point out differences with related work in the literature and discuss them. As an application of the obtained results, we evaluate the divergences of the vacuum expectation value of the Higgs field at one loop and show that they can be appropriately removed by the corresponding renormalization. We also work out the finite renormalization required to keep the no-tadpole condition on the Higgs field at one loop.
\end{abstract}

DOI: $10.1103 /$ PhysRevD.104.076005

\section{INTRODUCTION}

Exploring the Higgs sector at the Large Hadron Collider (LHC) requires a parametrization of Higgs-boson properties, able to account for effects beyond the Standard Model (SM) in a well-defined way. This is frequently done using the $\kappa$ framework, where SM couplings of the Higgs boson are rescaled by phenomenological factors [1]. The electroweak chiral Lagrangian including a light Higgs boson (EWChL for short) provides us with a systematic effective field theory (EFT) formulation of this idea [2] (see also [3] for a detailed discussion of the precise relationship between the $\kappa$ parameters and EWChL coefficients). The power counting of the EWChL is governed by chiral dimensions, equivalent to an expansion in loop orders. The leadingorder Lagrangian $\mathcal{L}_{2}$, of chiral dimension 2 , naturally contains the dominant anomalous Higgs couplings.

Several authors have contributed to the development of the chiral Lagrangian with a light Higgs boson as an EFT of electroweak symmetry breaking [4-13], which includes the complete classification of the next-to-leading-order (NLO)

Published by the American Physical Society under the terms of the Creative Commons Attribution 4.0 International license. Further distribution of this work must maintain attribution to the author(s) and the published article's title, journal citation, and DOI. Funded by SCOAP ${ }^{3}$. terms [14] and a systematic review of its power counting [15]. Motivated by the importance of the EWChL as an EFT of anomalous Higgs couplings, we have computed its complete one-loop renormalization in a previous article [16]. Similar work was reported shortly thereafter in [17]. The consideration of one-loop corrections is, in particular, needed when treating subleading effects, which are of interest for their impact on decay distributions.

Since the leading-order Lagrangian $\mathcal{L}_{2}$ of the EWChL is nonrenormalizable, the one-loop renormalization, besides affecting $\mathcal{L}_{2}$ itself, requires the addition of counterterms with chiral dimension 4 . In the present paper, we investigate the one-loop divergences of the electroweak chiral Lagrangian in detail. This includes their decomposition into a basis of counterterms, their renormalization, and the derivation of renormalization group equations (RGEs) for all parameters in explicit terms. This work parallels similar calculations done in the past in the context of chiral perturbation theory for pions and kaons coupled to photons [18] and light fermions [19], extending the original results of [20].

The outline of the present paper is as follows. We set up our notation in Sec. II. In Sec. III we decompose the oneloop divergences into the various classes of basis operators. We work out the renormalization of the leading-order Lagrangian and the next-to-leading-order counterterms in Secs. IV and V, respectively, deriving also the one-loop RGEs for all parameters of the theory. Section VI presents a 
comparison with the one-loop renormalization of SM effective field theory (SMEFT), which provides us with additional cross-checks of our results. A brief survey of the literature on the renormalization of the EWChL is given in Sec. VII. As an application, we treat the one-loop corrections to the vacuum expectation value (vev) of the Higgs field in Sec. VIII, showing how it is renormalized and computing the finite terms. We conclude in Sec. IX. Three appendixes are included. Appendix A collects the equations of motion, Appendix B the relevant basis operators at NLO, and Appendix C a summary of the one-loop divergences computed in [16].

\section{NOTATION AND LEADING-ORDER LAGRANGIAN}

In this paper we discuss the one-loop renormalization of the EWChL at lowest order, which can be written as $[14,15]$

$$
\begin{aligned}
\mathcal{L}_{2}= & -\frac{1}{4} G_{\mu \nu}^{\alpha} G^{\alpha \mu \nu}-\frac{1}{2}\left\langle W_{\mu \nu} W^{\mu \nu}\right\rangle-\frac{1}{4} B_{\mu \nu} B^{\mu \nu} \\
& +\frac{v^{2}}{4}\left\langle L_{\mu} L^{\mu}\right\rangle F\left(\frac{h}{v}\right)+\frac{1}{2} \partial_{\mu} h \partial^{\mu} h-V\left(\frac{h}{v}\right) \\
& +\bar{\psi} i \not D \psi-\bar{\psi} m(h / v, U) \psi,
\end{aligned}
$$

starting from the one-loop divergences obtained in Ref. [16]. In this expression, $G_{\mu}^{\alpha}, W_{\mu}^{a}$, and $B_{\mu}$ are the gauge fields of $S U(3)_{C}, S U(2)_{L}$, and $U(1)_{Y}$, respectively, $h$ denotes the Higgs field, and $v=246 \mathrm{GeV}$ is the electroweak scale. The $S U(2)$ trace is denoted by $\langle\ldots\rangle$.

The SM fermions are collected in the field $\psi=\left(u_{i}, d_{i}\right.$, $\left.\nu_{i}, e_{i}\right)^{T}$, where $u_{i}, d_{i}, \nu_{i}$, and $e_{i}$ are Dirac spinors and $i$ is the generation index. The covariant derivative on the fermion field reads

$D_{\mu} \psi=\left(\partial_{\mu}+i g_{s} G_{\mu}+i g W_{\mu} P_{L}+i g^{\prime} B_{\mu}\left(Y_{L} P_{L}+Y_{R} P_{R}\right)\right) \psi$,

where $P_{L}, P_{R}$ are the left and right chiral projectors and the weak hypercharge is described by the diagonal matrices

$$
\begin{aligned}
& Y_{L}=\operatorname{diag}(1 / 6,1 / 6,-1 / 2,-1 / 2), \\
& Y_{R}=\operatorname{diag}(2 / 3,-1 / 3,0,-1) .
\end{aligned}
$$

We will also use the notation $\psi_{L} \equiv P_{L} \psi, \psi_{R} \equiv P_{R} \psi$, and $q=\left(u_{i}, d_{i}\right)^{T}, l=\left(\nu_{i}, e_{i}\right)^{T}$.

The electroweak Goldstone bosons are parametrized as $U=\exp (2 i \varphi / v)$, where $\varphi=\varphi^{a} T^{a}$ and $T^{a}$ denote the generators of $S U(2)$, normalized as $\left\langle T^{a} T^{b}\right\rangle=\delta^{a b} / 2$. It is convenient to define their covariant derivative as

$$
\begin{aligned}
L_{\mu} & =i U D_{\mu} U^{\dagger}, \quad D_{\mu} U=\partial_{\mu} U+i g W_{\mu} U-i g^{\prime} B_{\mu} \tau_{L} U, \\
\tau_{L} & =U T^{3} U^{\dagger} .
\end{aligned}
$$

The Yukawa term has been compactly expressed through

$$
m(\eta, U) \equiv U \mathcal{M}(\eta) P_{R}+\mathcal{M}^{\dagger}(\eta) U^{\dagger} P_{L},
$$

where $\eta \equiv h / v$ and $\mathcal{M}$ is the block-diagonal mass matrix

$$
\mathcal{M}=\operatorname{diag}\left(\mathcal{M}_{u}, \mathcal{M}_{d}, \mathcal{M}_{\nu}, \mathcal{M}_{e}\right)
$$

acting on $\psi$. The entries $\mathcal{M}_{f} \equiv \mathcal{M}_{f}(\eta)$ are $h$-dependent matrices in generation space. We will take $\mathcal{M}_{\nu}=0$ in our calculation.

The Higgs-dependent functions can be expanded as

$$
\begin{aligned}
F(\eta) & =1+\sum_{n=1}^{\infty} F_{n} \eta^{n}, \quad V(\eta)=v^{4} \sum_{n=2}^{\infty} V_{n} \eta^{n}, \\
\mathcal{M}(\eta) & =\sum_{n=0}^{\infty} \mathcal{M}_{n} \eta^{n},
\end{aligned}
$$

where the potential is defined such that $V^{\prime}(0)=0$.

In order to express our results in a compact way we will use the combinations

$$
\kappa \equiv \frac{1}{2} F^{\prime} F^{-1 / 2}, \quad \mathcal{B} \equiv-F^{-1 / 2} \kappa^{\prime}=\frac{F^{2}}{4 F^{2}}-\frac{F^{\prime \prime}}{2 F} .
$$

Here and in the following, a prime on $\eta$-dependent functions denotes differentiation with respect to this variable. Of particular interest are the values of these functions for $\eta=0$,

$$
\begin{aligned}
F(0) & =1, \quad \kappa(0)=F_{1} / 2, \quad \mathcal{B}(0)=\frac{F_{1}^{2}}{4}-F_{2}, \\
V(0) & =V^{\prime}(0)=0, \quad V^{\prime \prime}(0)=2 v^{4} V_{2}=v^{2} m_{h}^{2}, \\
\mathcal{M}(0) & =\mathcal{M}_{0},
\end{aligned}
$$

and the deviations from these values,

$$
\begin{aligned}
\bar{F}(\eta) & =F(\eta)-1, \quad \bar{\kappa}(\eta)=\kappa(\eta)-F_{1} / 2, \\
\overline{\mathcal{B}} & =\mathcal{B}-\frac{F_{1}^{2}}{4}+F_{2}, \quad \overline{\mathcal{M}}=\mathcal{M}-\mathcal{M}_{0} .
\end{aligned}
$$

\section{DECOMPOSITION OF THE ONE-LOOP DIVERGENCES ON A BASIS OF COUNTERTERMS}

The first step will be to decompose the one-loop divergences worked out in Ref. [16] and project them onto the basis of one-loop counterterms derived in Ref. [14]. Some of the divergences displayed in Ref. [16] actually have the same structure as the lowest-order effective Lagrangian $\mathcal{L}_{2}$, and are thus dealt with by means of a set of counterterms $\Delta \mathcal{L}_{2}$, involving only the structures already present in $\mathcal{L}_{2}$. 
It is convenient to decompose the one-loop divergent parts according to the presence of spin-one field strengths, scalar fields, or fermion fields as

$$
\mathcal{L}_{\text {div }}=\mathcal{L}_{\text {div }}^{(1)}+\mathcal{L}_{\text {div }}^{(0)}+\mathcal{L}_{\text {div }}^{(1 / 2)}
$$

where the explicit expressions for each term on the righthand side can be found in Eqs. (C1), (C2), and (C11), respectively, in Appendix C. We proceed with each term in turn.

In manipulating the one-loop divergent pieces, one is entitled to make use of the equations of motion at leading order, which are collected in Appendix A. One reason for this is that the divergences are expressed in terms of the classical background fields. One may thus use the classical, lowest-order, equations of motion as long as the corresponding counterterms are inserted into tree-level diagrams only, which is the case for the computation of amplitudes at next-to-leading accuracy. More generally, the use of the equations of motion can also be implemented as a field redefinition, which does not change the S-matrix elements. For a general discussion on this issue, see e.g., Ref. [21,22]. As a practical consequence, it is therefore not necessary to introduce next-to-leading counterterms corresponding to the field renormalizations for fermions and the Higgs boson.

In the results collected in Eqs. (C1), (C2), and (C3), which are taken from Ref. [16], the equations of motion had already been used for the scalar fields $U$ and $h$. In Appendix $\mathrm{C}$ we extend this to fermions.

\section{A. Decomposition of $\mathcal{L}_{\text {div }}^{(1)}$}

From Eq. (C1), one has $\left(N_{c}\right.$ stands for the number of colors, $N_{f}$ for the number of quark flavors, and $N_{\mathrm{g}}$ for the number of generations)

$$
\begin{aligned}
\mathcal{L}_{\mathrm{div}}^{(1)}= & -\frac{1}{16 \pi^{2}} \frac{1}{d-4}\left\{\frac{g_{s}^{2}}{2} G^{\alpha \mu \nu} G_{\mu \nu}^{\alpha}\left(\frac{11}{3} N_{c}-\frac{2}{3} N_{f}\right)+\frac{g^{2}}{2}\left\langle W^{\mu \nu} W_{\mu \nu}\right\rangle\left[\frac{43}{3}-\frac{2}{3}\left(N_{c}+1\right) N_{\mathrm{g}}-\frac{F_{1}^{2}-4}{24}\right]\right. \\
& +\frac{g^{\prime 2}}{2} B^{\mu \nu} B_{\mu \nu}\left[-\left(\frac{11}{27} N_{c}+1\right) N_{\mathrm{g}}-\frac{1}{6}-\frac{F_{1}^{2}-4}{48}\right]-\frac{1}{24} \bar{\kappa}\left(\bar{\kappa}+F_{1}\right)\left[g^{\prime 2} B^{\mu \nu} B_{\mu \nu}+2 g^{2}\left\langle W^{\mu \nu} W_{\mu \nu}\right\rangle\right] \\
& -i \frac{\bar{\kappa}\left(\bar{\kappa}+F_{1}\right)}{12}\left(g\left\langle W^{\mu \nu}\left[L_{\mu}, L_{\nu}\right]\right\rangle+g^{\prime} B^{\mu \nu}\left\langle\tau_{L}\left[L_{\mu}, L_{\nu}\right]\right\rangle+2 i g g^{\prime}\left\langle\tau_{L} W^{\mu \nu}\right\rangle B_{\mu \nu}\right) \\
& -i \frac{F_{1}^{2}-4}{48}\left(g\left\langle W^{\mu \nu}\left[L_{\mu}, L_{\nu}\right]\right\rangle+g^{\prime} B^{\mu \nu}\left\langle\tau_{L}\left[L_{\mu}, L_{\nu}\right]\right\rangle+2 i g g^{\prime}\left\langle\tau_{L} W^{\mu \nu}\right\rangle B_{\mu \nu}\right) \\
& \left.-\frac{1}{6} \partial^{\mu}\left(\kappa^{2}\right)\left(g\left\langle W_{\mu \nu} L^{\nu}\right\rangle-g^{\prime} B_{\mu \nu}\left\langle\tau_{L} L^{\nu}\right\rangle\right)\right\},
\end{aligned}
$$

where we have separated explicitly terms with and without Higgs field dependence.

It is tempting to read off the one-loop beta functions for the gauge couplings directly from the coefficients of the gauge kinetic terms, as given by the first three terms above. However, in the case of the $S U(2)$ and $U(1)$ gauge couplings, one first needs to specify their definition, which, at one-loop order, depends on the choice of NLO operators. In the basis of [14], which we will use in this paper, the first two operators in the fourth line are absent. They can be eliminated from Eq. (3.2) by using the relations

$$
\begin{gathered}
\left\langle W^{\mu \nu}\left[L_{\mu}, L_{\nu}\right]\right\rangle=i g\left\langle W^{\mu \nu} W_{\mu \nu}\right\rangle-i g^{\prime} B^{\mu \nu}\left\langle\tau_{L} W_{\mu \nu}\right\rangle+i g \bar{\psi}_{L} \not \psi_{L}-i g \frac{v^{2}}{2} F(\eta)\left\langle L^{\nu} L_{\nu}\right\rangle, \\
B^{\mu \nu}\left\langle\tau_{L}\left[L_{\mu}, L_{\nu}\right]\right\rangle=\frac{i g^{\prime}}{2} B^{\mu \nu} B_{\mu \nu}-i g B^{\mu \nu}\left\langle\tau_{L} W_{\mu \nu}\right\rangle-2 i g^{\prime}\left\langle\tau_{L} L^{\nu}\right\rangle\left(\bar{\psi} \gamma_{\nu}\left(Y_{L} P_{L}+Y_{R} P_{R}\right) \psi+\frac{v^{2}}{2} F(\eta)\left\langle L_{\nu} \tau_{L}\right\rangle\right),
\end{gathered}
$$

which follow from the identities [14]

$$
\begin{aligned}
D_{\mu} L_{\nu}-D_{\nu} L_{\mu} & =g W_{\mu \nu}-g^{\prime} B_{\mu \nu} \tau_{L}+i\left[L_{\mu}, L_{\nu}\right], \quad D_{\mu} \tau_{L}=i\left[L_{\mu}, \tau_{L}\right], \\
\left\langle T^{a} L^{\nu}\right\rangle\left\langle T^{a} L_{\nu}\right\rangle & =\frac{1}{2}\left\langle L^{\nu} L_{\nu}\right\rangle, \quad\left\langle L^{\nu} T^{a}\right\rangle \bar{\psi}_{L} \gamma_{\nu} T^{a} \psi_{L}=\frac{1}{2} \bar{\psi}_{L} \not \psi_{L},
\end{aligned}
$$

from the equations of motion given in Appendix A, and from integrating by parts. Because of the last point, (3.3) and (3.4) can only be used in the form given here if the operators on the left-hand side are not multiplied by $h$-dependent functions. The expression of $\mathcal{L}_{\text {div }}^{(1)}$ then can be rewritten as 


$$
\begin{aligned}
\mathcal{L}_{\text {div }}^{(1)}= & -\frac{1}{16 \pi^{2}} \frac{1}{d-4}\left\{\frac{g_{s}^{2}}{2} G^{\alpha \mu \nu} G_{\mu \nu}^{\alpha}\left(\frac{11}{3} N_{c}-\frac{2}{3} N_{f}\right)+\frac{g^{2}}{2}\left\langle W^{\mu \nu} W_{\mu \nu}\right\rangle\left[\frac{43}{3}-\frac{2}{3}\left(N_{c}+1\right) N_{\mathrm{g}}\right]\right. \\
& +\frac{g^{\prime 2}}{2} B^{\mu \nu} B_{\mu \nu}\left[-\left(\frac{11}{27} N_{c}+1\right) N_{\mathrm{g}}-\frac{1}{6}\right]-\frac{1}{24} \bar{\kappa}\left(\bar{\kappa}+F_{1}\right)\left[g^{\prime 2} B^{\mu \nu} B_{\mu \nu}+2 g^{2}\left\langle W^{\mu \nu} W_{\mu \nu}\right\rangle\right] \\
& -i \frac{\bar{\kappa}\left(\bar{\kappa}+F_{1}\right)}{12}\left(g\left\langle W^{\mu \nu}\left[L_{\mu}, L_{\nu}\right]\right\rangle+g^{\prime} B^{\mu \nu}\left\langle\tau_{L}\left[L_{\mu}, L_{\nu}\right]\right\rangle+2 i g g^{\prime}\left\langle\tau_{L} W^{\mu \nu}\right\rangle B_{\mu \nu}\right) \\
& +\frac{F_{1}^{2}-4}{48}\left[g^{2} \bar{\psi}_{L} \not\left\langle\psi_{L}-g^{2} \frac{v^{2}}{2} F(\eta)\left\langle L^{\nu} L_{\nu}\right\rangle-2 g^{\prime 2}\left\langle\tau_{L} L^{\nu}\right\rangle\left(\bar{\psi} \gamma_{\nu}\left(Y_{L} P_{L}+Y_{R} P_{R}\right) \psi+\frac{v^{2}}{2} F(\eta)\left\langle L_{\nu} \tau_{L}\right\rangle\right)\right]\right. \\
& \left.-\frac{1}{6} \partial^{\mu}\left(\kappa^{2}\right)\left(g\left\langle W_{\mu \nu} L^{\nu}\right\rangle-g^{\prime} B_{\mu \nu}\left\langle\tau_{L} L^{\nu}\right\rangle\right)\right\} .
\end{aligned}
$$

In (3.6), the operators that are redundant with respect to the basis of [14] have been eliminated. The gauge beta functions defined by this NLO basis can then be read off from the coefficients of the gauge kinetic terms. By inspection of (3.6) we find that the one-loop gauge beta functions actually coincide with the expressions obtained in the SM. The use of redundancy relations like (3.3) and (3.4), which contain both genuine NLO structures and the gauge kinetic operators, in changing to a different NLO basis, implies redefinitions of the gauge fields, the couplings and their beta functions.

We next consider the two terms proportional to $\partial^{\mu}\left(\kappa^{2}\right)$ on the last line of the above expression. At this stage, we will need the equations of motion of the electroweak gauge fields, given in Eqs. (A1) and (A2), as well as the identities of Eq. (3.5). Up to total-derivative terms, which are discarded, we obtain

$\partial^{\mu}\left(\kappa^{2}\right)\left\langle W_{\mu \nu} L^{\nu}\right\rangle=-\frac{\bar{\kappa}\left(\bar{\kappa}+F_{1}\right)}{2} g\left[-\frac{v^{2}}{2} F(\eta)\left\langle L^{\nu} L_{\nu}\right\rangle+\bar{\psi}_{L} \not \psi_{L}\right]-\frac{\bar{\kappa}\left(\bar{\kappa}+F_{1}\right)}{2}\left[g\left\langle W^{\mu \nu} W_{\mu \nu}\right\rangle-g^{\prime} B^{\mu \nu}\left\langle W_{\mu \nu} \tau_{L}\right\rangle+i\left\langle W_{\mu \nu}\left[L^{\mu}, L^{\nu}\right]\right\rangle\right]$,

and

$$
\begin{aligned}
\partial^{\mu}\left(\kappa^{2}\right) B_{\mu \nu}\left\langle\tau_{L} L^{\nu}\right\rangle= & -\bar{\kappa}\left(\bar{\kappa}+F_{1}\right) g^{\prime}\left[\frac{v^{2}}{2}\left\langle L_{\nu} \tau_{L}\right\rangle F(\eta)+\bar{\psi} \gamma_{\nu}\left(Y_{L} P_{L}+Y_{R} P_{R}\right) \psi\right]\left\langle\tau_{L} L^{\nu}\right\rangle \\
& +\frac{\bar{\kappa}\left(\bar{\kappa}+F_{1}\right)}{2} B_{\mu \nu}\left\langle i \tau_{L}\left[L^{\mu}, L^{\nu}\right]-g \tau_{L} W^{\mu \nu}+g^{\prime} \tau_{L}^{2} B^{\mu \nu}\right\rangle
\end{aligned}
$$

Making the corresponding substitutions in the above expression of $\mathcal{L}_{\text {div }}^{(1)}$, we find

$$
\begin{aligned}
\mathcal{L}_{\text {div }}^{(1)}= & -\frac{1}{16 \pi^{2}} \frac{1}{d-4}\left\{\frac{g_{s}^{2}}{2} G^{\alpha \mu \nu} G_{\mu \nu}^{\alpha}\left(\frac{11}{3} N_{c}-\frac{2}{3} N_{f}\right)+\frac{g^{2}}{2}\left\langle W^{\mu \nu} W_{\mu \nu}\right\rangle\left[\frac{43}{3}-\frac{2}{3}\left(N_{c}+1\right) N_{\mathrm{g}}\right]\right. \\
& +\frac{g^{\prime 2}}{2} B^{\mu \nu} B_{\mu \nu}\left[-\left(\frac{11}{27} N_{c}+1\right) N_{\mathrm{g}}-\frac{1}{6}\right]-g^{2} \frac{v^{2}}{24}\left(\kappa^{2}-1\right) F(\eta)\left\langle L^{\nu} L_{\nu}\right\rangle \\
& \left.+\frac{\kappa^{2}-1}{12} g^{2} \bar{\psi}_{L} \not L \psi_{L}-\frac{\kappa^{2}-1}{6} g^{\prime 2}\left[\frac{v^{2}}{2}\left\langle L_{\nu} \tau_{L}\right\rangle F(\eta)+\bar{\psi} \gamma_{\nu}\left(Y_{L} P_{L}+Y_{R} P_{R}\right) \psi\right]\left\langle\tau_{L} L^{\nu}\right\rangle\right\} \\
\equiv & \Delta^{(1)} \mathcal{L}_{2}+\Delta^{(1)} \mathcal{L}_{\beta_{1}}+\Delta^{(1)} \mathcal{L}_{\psi^{2} U h D} .
\end{aligned}
$$

The term

$$
\begin{aligned}
\Delta^{(1)} \mathcal{L}_{2}= & -\frac{1}{16 \pi^{2}} \frac{1}{d-4}\left\{-\frac{g_{s}^{2}}{4} G^{\alpha \mu \nu} G_{\mu \nu}^{\alpha}\left(-\frac{22}{3} N_{c}+\frac{4}{3} N_{f}\right)-\frac{g^{2}}{2}\left\langle W^{\mu \nu} W_{\mu \nu}\right\rangle\left[-\frac{44}{3}+\frac{2}{3}\left(N_{c}+1\right) N_{\mathrm{g}}+\frac{1}{3}\right]\right. \\
& \left.-\frac{g^{\prime 2}}{4} B^{\mu \nu} B_{\mu \nu}\left[2\left(\frac{11}{27} N_{c}+1\right) N_{\mathrm{g}}+\frac{1}{3}\right]-g^{2} \frac{v^{2}}{24}\left(\kappa^{2}-1\right) F(\eta)\left\langle L_{\mu} L^{\mu}\right\rangle\right\}
\end{aligned}
$$

renormalizes the gauge-field and Goldstone-boson kinetic terms in $\mathcal{L}_{2}$. The two last terms of $\mathcal{L}_{\text {div }}^{(1)}$, to which we will return later, read 


$$
\Delta^{(1)} \mathcal{L}_{\beta_{1}}=-\frac{1}{16 \pi^{2}} \frac{1}{d-4} \times g^{\prime 2} \frac{v^{2}}{2} F(\eta) \frac{1-\kappa^{2}}{6}\left\langle L^{\nu} \tau_{L}\right\rangle\left\langle L_{\nu} \tau_{L}\right\rangle
$$

and

$$
\Delta^{(1)} \mathcal{L}_{\psi^{2} U h D}=-\frac{1}{16 \pi^{2}} \frac{1}{d-4} \frac{\kappa^{2}-1}{12}\left\{g^{2} \bar{\psi}_{L} \not L \psi_{L}-2 g^{\prime 2}\left\langle\tau_{L} L^{\nu}\right\rangle \bar{\psi} \gamma_{\nu}\left(Y_{L} P_{L}+Y_{R} P_{R}\right) \psi\right\}
$$

$\Delta^{(1)} \mathcal{L}_{\beta_{1}}$ renormalizes the custodial-symmetry breaking operator $\mathcal{L}_{\beta_{1}}$, while $\Delta^{(1)} \mathcal{L}_{\psi^{2} U h D}$ renormalizes some of the counterterms of the class $\psi^{2} U h D$, respectively. We refer to Appendix B for the detailed definition of operators and their classes. We employ the nomenclature of Ref. [14], which will be used throughout. Incidentally, we note that the divergences involving the next-to-leading-order operators of the classes $X^{2} h$ and $X U h D^{2}$ have canceled.

Finally, in order to bring all the terms in correspondence to the operator basis retained in Ref. [14], the quantities of the type $\bar{\psi}_{L} O_{1} \not O_{2} \psi_{L}$ can be transformed as follows $\left(P_{12}=T^{1}+i T^{2}, P_{21}=T^{1}-i T^{2}\right)$ :

$$
\begin{aligned}
\bar{\psi}_{L} O_{1} \not L O_{2} \psi_{L} & =2 \bar{\psi}_{L} \gamma^{\mu} O_{1} U T^{a} U^{\dagger} O_{2} \psi_{L}\left\langle U T^{a} U^{\dagger} L_{\mu}\right\rangle \\
& =\bar{\psi}_{L} \gamma^{\mu} O_{1} U P_{12} U^{\dagger} O_{2} \psi_{L}\left\langle U P_{21} U^{\dagger} L_{\mu}\right\rangle+\bar{\psi}_{L} \gamma^{\mu} O_{1} U P_{21} U^{\dagger} O_{2} \psi_{L}\left\langle U P_{12} U^{\dagger} L_{\mu}\right\rangle+2 \bar{\psi}_{L} \gamma^{\mu} O_{1} \tau_{L} O_{2} \psi_{L}\left\langle\tau_{L} L_{\mu}\right\rangle .
\end{aligned}
$$

Using the previous relation, we find

$$
\begin{aligned}
\Delta^{(1)} \mathcal{L}_{\psi^{2} U h D}= & -\frac{1}{16 \pi^{2}} \frac{1}{d-4} \frac{\kappa^{2}-1}{12}\left\{2 g^{2} \bar{\psi}_{L} \tau_{L} \gamma^{\mu} \psi_{L}\left\langle\tau_{L} L_{\mu}\right\rangle+g^{2} \bar{\psi}_{L} U P_{12} U^{\dagger} \gamma^{\mu} \psi_{L}\left\langle U P_{21} U^{\dagger} L_{\mu}\right\rangle\right. \\
& +g^{2} \bar{\psi}_{L} U P_{21} U^{\dagger} \gamma^{\mu} \psi_{L}\left\langle U P_{12} U^{\dagger} L_{\mu}\right\rangle-2 g^{\prime 2}\left\langle\tau_{L} L^{\nu}\right\rangle \bar{\psi}_{L} \gamma_{\nu} Y_{L} \psi_{L}-\frac{4}{3} g^{2}\left\langle\tau_{L} L^{\nu}\right\rangle \bar{u}_{R} \gamma_{\nu} u_{R} \\
& \left.+\frac{2}{3} g^{\prime 2}\left\langle\tau_{L} L^{\nu}\right\rangle \bar{d}_{R} \gamma_{\nu} d_{R}+2 g^{\prime 2}\left\langle\tau_{L} L^{\nu}\right\rangle \bar{e}_{R} \gamma_{\nu} e_{R}\right\} .
\end{aligned}
$$

\section{B. Decomposition of $\mathcal{L}_{\text {div }}^{(0)}$}

We consider next the divergent pieces involving spin-zero fields given in Eq. (C2), which we rewrite as

$$
\mathcal{L}_{\text {div }}^{(0)}=\Delta^{(0)} \mathcal{L}_{2}+\Delta^{(0)} \mathcal{L}_{U h D^{4}}+\Delta^{(0)} \mathcal{L}_{\beta_{1}}
$$

where $(\langle\ldots\rangle\rangle$ denotes a trace over isospin, as well as generations and color)

$$
\begin{aligned}
\Delta^{(0)} \mathcal{L}_{2}= & -\frac{1}{16 \pi^{2}} \frac{1}{d-4}\left\{\frac { v ^ { 2 } } { 4 } \left[-\frac{g^{\prime 2}}{8}\left(F_{1}^{2}+12\right)-\frac{3 g^{2}}{4}\left(F_{1}^{2}+4\right)+V_{2}\left(F_{1}^{2}-4 F_{2}\right)+\frac{4}{v^{2}}\left\langle\left\langle\mathcal{M}_{0}^{\dagger} \mathcal{M}_{0}\right\rangle\right\rangle\right.\right. \\
& -\frac{g^{\prime 2}}{8}\left(F_{1}^{2}+12\right) \bar{F}-\frac{g^{\prime 2}}{2} \bar{\kappa}\left(\bar{\kappa}+F_{1}\right) F-\frac{3 g^{2}}{4}\left(F_{1}^{2}+4\right) \bar{F}-3 g^{2} \bar{\kappa}\left(\bar{\kappa}+F_{1}\right) F \\
& -2\left(\kappa^{2}-1\right) \frac{F^{\prime} V^{\prime}}{F v^{4}}+2 \frac{\left(V^{\prime \prime}-2 v^{4} V_{2}\right)}{v^{4}} F \mathcal{B}+4 V_{2}(\mathcal{B} \bar{F}+\overline{\mathcal{B}})+\frac{4}{v^{2}}\left\langle\left\langle\overline{\mathcal{M}}^{\dagger} \mathcal{M}_{0}+\mathcal{M}_{0}^{\dagger} \overline{\mathcal{M}}+\overline{\mathcal{M}} \dagger \overline{\mathcal{M}}\right\rangle\right\rangle\left\langle L^{\mu} L_{\mu}\right\rangle \\
& +\frac{1}{2}\left[-\frac{3 F_{1}^{2}+4 F_{2}}{8}\left(3 g^{2}+g^{\prime 2}\right)+\frac{4}{v^{2}}\left\langle\left\langle\mathcal{M}_{1}^{\dagger} \mathcal{M}_{1}\right\rangle\right\rangle\right. \\
& \left.+\frac{1}{2}\left(3 g^{2}+g^{\prime 2}\right)\left(\bar{F} \mathcal{B}+\overline{\mathcal{B}}-4 \bar{\kappa}^{2}-4 F_{1} \bar{\kappa}\right)+3 \frac{F^{\prime} V^{\prime}}{F v^{4}} \mathcal{B}+\frac{4}{v^{2}}\left\langle\left\langle\overline{\mathcal{M}}^{\prime \dagger} \mathcal{M}_{1}+\mathcal{M}_{1}^{\dagger} \overline{\mathcal{M}}^{\prime}+\overline{\mathcal{M}}^{\prime \dagger} \overline{\mathcal{M}}^{\prime}\right\rangle\right\rangle\right] \partial^{\mu} h \partial_{\mu} h \\
& +\frac{3}{2}\left(3 g^{4}+2 g^{2} g^{\prime 2}+g^{\prime 4}\right) \frac{v^{4}}{16} F^{2}+\frac{3 g^{2}+g^{\prime 2}}{8} F^{\prime} V^{\prime}+\frac{3}{8}\left(\frac{F^{\prime} V^{\prime}}{F v^{2}}\right)^{2}+\frac{1}{2}\left(\frac{V^{\prime \prime}}{v^{2}}\right)^{2}-2\left\langle\left\langle\left(\mathcal{M}^{\dagger} \mathcal{M}^{2}\right\rangle\right\rangle\right. \\
& \left.+4 i\left\langle\tau_{L} L_{\mu}\right\rangle\left\langle\left\langle\left(\partial^{\mu} \mathcal{M}^{\dagger} \mathcal{M}-\mathcal{M}^{\dagger} \partial^{\mu} \mathcal{M}\right) T^{3}\right\rangle\right\rangle\right\},
\end{aligned}
$$




$$
\begin{aligned}
& \Delta^{(0)} \mathcal{L}_{U h D^{4}}=-\frac{1}{16 \pi^{2}} \frac{1}{d-4}\left\{\frac{\left(\kappa^{2}-1\right)^{2}}{6}\left\langle L_{\mu} L_{\nu}\right\rangle\left\langle L^{\mu} L^{\nu}\right\rangle+\left(\frac{\left(\kappa^{2}-1\right)^{2}}{12}+\frac{F^{2} \mathcal{B}^{2}}{8}\right)\left\langle L^{\mu} L_{\mu}\right\rangle^{2}+\frac{2}{3} \kappa^{\prime 2}\left\langle L_{\mu} L_{\nu}\right\rangle \partial^{\mu} \eta \partial^{\nu} \eta\right. \\
&\left.-\left(\left(\kappa^{2}-1\right) \mathcal{B}+\frac{\kappa^{\prime 2}}{6}\right)\left\langle L^{\mu} L_{\mu}\right\rangle \partial^{\nu} \eta \partial_{\nu} \eta+\frac{3}{2} \mathcal{B}^{2}\left(\partial^{\mu} \eta \partial_{\mu} \eta\right)^{2}\right\}, \\
& \Delta^{(0)} \mathcal{L}_{\beta_{1}}=-\frac{1}{16 \pi^{2}} \frac{1}{d-4} \times \frac{3}{4} g^{\prime 2} v^{2}\left(1-\kappa^{2}\right) F\left\langle\tau_{L} L^{\mu}\right\rangle\left\langle\tau_{L} L_{\mu}\right\rangle .
\end{aligned}
$$

The two last terms renormalize the counterterms of the class $U h D^{4}$ and the custodial-symmetry breaking operator $\mathcal{L}_{\beta_{1}}$, respectively. $\Delta^{(0)} \mathcal{L}_{U h D^{4}}$ comes already fully expressed in terms of the basis elements displayed in Ref. [14].

The last term of Eq. (3.16) does not naturally appear as a renormalization of $\mathcal{L}_{2}$, but can be shown to renormalize the Yukawa term. Using the equation of motion for $B_{\mu}$ in (A1), one may write

$$
\left.\left.4 i\left\langle\tau_{L} L_{\mu}\right\rangle\left\langle\left(\partial^{\mu} \mathcal{M}^{\dagger} \mathcal{M}-\mathcal{M}^{\dagger} \partial^{\mu} \mathcal{M}\right) T^{3}\right\rangle\right\rangle=\frac{8 i}{v^{2} F}\left[\frac{1}{g^{\prime}} \partial^{\nu} B_{\nu \mu}-\bar{\psi} \gamma_{\mu}\left(Y_{L} P_{L}+Y_{R} P_{R}\right) \psi\right]\left\langle\left(\partial^{\mu} \mathcal{M}^{\dagger} \mathcal{M}-\mathcal{M}^{\dagger} \partial^{\mu} \mathcal{M}\right) T^{3}\right\rangle\right\rangle .
$$

The first term between square brackets on the right-hand side of this relation leads to a total derivative,

$$
\begin{aligned}
F^{-1} \partial^{\nu} B_{\nu \mu}\left\langle\left\langle\left(\partial^{\mu} \mathcal{M}^{\dagger} \mathcal{M}-\mathcal{M}^{\dagger} \partial^{\mu} \mathcal{M}\right) T^{3}\right\rangle\right\rangle= & \left.\partial^{\nu}\left[F^{-1} B_{\nu \mu}\left\langle\left(\partial^{\mu} \mathcal{M}^{\dagger} \mathcal{M}-\mathcal{M}^{\dagger} \partial^{\mu} \mathcal{M}\right) T^{3}\right\rangle\right\rangle\right] \\
& -B_{\nu \mu} \partial^{\nu}\left[F^{-1}\left\langle\left(\partial^{\mu} \mathcal{M}^{\dagger} \mathcal{M}-\mathcal{M}^{\dagger} \partial^{\mu} \mathcal{M}\right) T^{3}\right\rangle\right],
\end{aligned}
$$

since the second term in the above relation vanishes, being proportional either to $B_{\mu \nu}\left(\partial^{\mu} \eta\right)\left(\partial^{\nu} \eta\right)$ or to $B_{\mu \nu}\left(\partial^{\mu} \partial^{\nu} \eta\right)$. One thus ends up with

$$
\begin{aligned}
4 i\left\langle\tau_{L} L_{\mu}\right\rangle\left\langle\left\langle\left(\partial^{\mu} \mathcal{M}^{\dagger} \mathcal{M}-\mathcal{M}^{\dagger} \partial^{\mu} \mathcal{M}\right) T^{3}\right\rangle\right\rangle \\
\quad=\frac{8 i}{v^{2}}\left\langle\left\langle\left(F^{-1 / 2} \mathcal{M}^{\dagger} \partial^{\mu}\left(F^{-1 / 2} \mathcal{M}\right)-\partial^{\mu}\left(F^{-1 / 2} \mathcal{M}^{\dagger}\right) F^{-1 / 2} \mathcal{M}\right) T^{3}\right\rangle \times \bar{\psi} \gamma_{\mu}\left(Y_{L} P_{L}+Y_{R} P_{R}\right) \psi\right. \\
\quad=\frac{8 i}{v^{2}}\left\langle\left\langle\left(F^{-1 / 2} \mathcal{M}^{\dagger}\left(F^{-1 / 2} \mathcal{M}\right)^{\prime}-\left(F^{-1 / 2} \mathcal{M}^{\dagger}\right)^{\prime} F^{-1 / 2} \mathcal{M}\right) T^{3}\right\rangle \times\left(\partial^{\mu} \eta\right) \bar{\psi} \gamma_{\mu}\left(Y_{L} P_{L}+Y_{R} P_{R}\right) \psi\right. \\
\quad=\frac{8 i}{v^{2}} \bar{\psi} \gamma_{\mu}\left(Y_{L} P_{L}+Y_{R} P_{R}\right) \psi \partial^{\mu} \int_{0}^{\eta} d s\left\langle\left\langle\left(F^{-1 / 2} \mathcal{M}^{\dagger}\left(F^{-1 / 2} \mathcal{M}\right)^{\prime}-\left(F^{-1 / 2} \mathcal{M}^{\dagger}\right)^{\prime} F^{-1 / 2} \mathcal{M}\right) T^{3}\right\rangle\right\rangle \\
\left.\quad=\frac{8}{v^{2}} \bar{\psi}\left[U \mathcal{M}\left(Y_{R}-Y_{L}\right) P_{R}-\left(Y_{R}-Y_{L}\right) \mathcal{M}^{\dagger} U^{\dagger} P_{L}\right] \psi \int_{0}^{\eta} d s\left\langle F^{-1 / 2} \mathcal{M}^{\dagger}\left(F^{-1 / 2} \mathcal{M}\right)^{\prime} T^{3}-\left(F^{-1 / 2} \mathcal{M}^{\dagger}\right)^{\prime} F^{-1 / 2} \mathcal{M} T^{3}\right\rangle\right\rangle .
\end{aligned}
$$

Here the integration variable $s$ has been introduced, which denotes the dependence of the integrands on $h / v=s$. In the last step, an integration by parts has been performed, the resulting total-derivative term has been dropped, and the equations of motion for the fermionic fields have been used. Notice that $Y_{R}-Y_{L}=T^{3}$. Objects like

$$
\int_{0}^{\eta} d s\left[F^{-1 / 2} \mathcal{M}^{\dagger}\left(F^{-1 / 2} \mathcal{M}\right)^{\prime}-\left(F^{-1 / 2} \mathcal{M}^{\dagger}\right)^{\prime} F^{-1 / 2} \mathcal{M}\right]
$$

are perfectly well defined as formal power series in $\eta$, obtained upon multiplication of the formal series defining, e.g., $F^{-1 / 2}(s) \mathcal{M}^{\dagger}(s)$ and $\left(F^{-1 / 2}(s) \mathcal{M}(s)\right)^{\prime}$, and term-by-term integration. ${ }^{1}$

\footnotetext{
${ }^{1}$ More precisely, given a local functional $\mathcal{F}(\eta(x))$ defined as a formal series, $\mathcal{F}(\eta(x)) \equiv \sum_{n \geq 0} \mathcal{F}_{n} \eta^{n}(x)$, we may define a primitive as the local functional $\hat{\mathcal{F}}(\eta(x)) \equiv \sum_{n \geq 1} \mathcal{F}_{n-1} \eta^{n}(x) / n$. This primitive may be obtained upon considering the function $\mathcal{F}(s)$, where $s$ is a real variable, defined by the same power series with coefficients $\mathcal{F}_{n}$ as $\mathcal{F}(\eta(x))$, and upon performing a term-by-term integration,

$$
\hat{\mathcal{F}}(\eta(x))=\int_{0}^{\eta(x)} d s \mathcal{F}(s) .
$$

Then one of course has $\partial_{\mu} \int_{0}^{\eta(x)} d s \mathcal{F}(s)=\left(\partial_{\mu} \eta(x)\right) \mathcal{F}(\eta(x))$. Most of the time we will omit the variable of integration $s$ and write $\int_{0}^{\eta} d s \mathcal{F}$ instead of $\int_{0}^{\eta(x)} d s \mathcal{F}(s)$. No confusion should arise from using this simplified notation.
} 


\section{Decomposition of $\mathcal{L}_{\text {div }}^{(1 / 2)}$}

Turning finally to the divergences involving also fermionic fields, Eq. (C11) gives

$$
\begin{aligned}
& \mathcal{L}_{\mathrm{div}}^{(1 / 2)}=-\frac{1}{16 \pi^{2}} \frac{1}{d-4}\left\{\bar{\psi}_{L}\left(\frac{3}{2} g^{2}+2 g^{\prime 2} Y_{L}^{2}\right) i \not D \psi_{L}+\bar{\psi}_{R} 2 g^{\prime 2} Y_{R}^{2} i \not D \psi_{R}+2 g_{s}^{2} C_{F} \bar{q}\left(i \not D-4\left(U \mathcal{M}_{q} P_{R}+\mathcal{M}_{q}^{\dagger} U^{\dagger} P_{L}\right)\right) q\right. \\
& +\frac{V^{\prime \prime}}{v^{4}}\left(\bar{\psi}_{L} U \mathcal{M}^{\prime \prime} \psi_{R}+\text { H.c. }\right)-8 g^{2}\left(\bar{\psi}_{L} Y_{L} U \mathcal{M} Y_{R} \psi_{R}+\text { H.c. }\right) \\
& +\left(\left(3 g^{2}+g^{\prime 2}\right) \frac{v^{2}}{4} F+\frac{3}{2} \frac{F^{\prime} V^{\prime}}{F v^{2}}\right) \frac{F^{-1}}{v^{2}}\left(\bar{\psi}_{L} U\left(\frac{F^{\prime}}{2} \mathcal{M}^{\prime}-\mathcal{M}\right) \psi_{R}+\text { H.c. }\right) \\
& +\frac{3}{v^{2}} F^{-1}\left(\bar{\psi}_{L} U \mathcal{M} \mathcal{M}^{\dagger} \mathcal{M} \psi_{R}+\text { H.c. }\right)-\frac{3}{v^{2}} F^{-1}\left(\bar{\psi}_{L} U\left\langle\mathcal{M} \mathcal{M}^{\dagger}\right\rangle \mathcal{M} \psi_{R}+\text { H.c. }\right) \\
& +\frac{2}{v^{2}}\left(\bar{\psi}_{L} U \mathcal{M}^{\prime} \mathcal{M}^{\dagger} \mathcal{M}^{\prime} \psi_{R}+\text { H.c. }\right)+\frac{1}{2 v^{2}}\left(\bar{\psi}_{L} U \mathcal{M} \mathcal{M}^{\prime \dagger} \mathcal{M}^{\prime} \psi_{R}+\text { H.c. }\right)+\frac{1}{2 v^{2}}\left(\bar{\psi}_{L} U \mathcal{M}^{\prime} \mathcal{M}^{\prime \dagger} \mathcal{M} \psi_{R}+\text { H.c. }\right) \\
& +\frac{3 F^{-1}}{2 v^{2}} \bar{\psi}_{R}\left(\mathcal{M}^{\dagger} i \not \partial \mathcal{M}-i \not \partial \mathcal{M}^{\dagger} \mathcal{M}\right) \psi_{R}+\frac{1}{2 v^{2}} \bar{\psi}_{R}\left(\mathcal{M}^{\dagger \dagger} i \not \partial \mathcal{M}^{\prime}-i \not \partial \mathcal{M}^{\prime \dagger} \mathcal{M}^{\prime}\right) \psi_{R} \\
& -\frac{F^{-1}}{2 v^{2}} \bar{\psi}_{L} U\left(\mathcal{M} i \not \partial \mathcal{M}^{\dagger}-i \not \partial \mathcal{M} \mathcal{M}^{\dagger}\right) U^{\dagger} \psi_{L}+\frac{1}{2 v^{2}} \bar{\psi}_{L} U\left(\mathcal{M}^{\prime} i \not \partial \mathcal{M}^{\prime \dagger}-i \not \partial \mathcal{M}^{\prime} \mathcal{M}^{\prime \dagger}\right) U^{\dagger} \psi_{L} \\
& +\frac{F^{-1}}{v^{2}} \bar{\psi}_{L}\left\langle\mathcal{M} i \not \partial \mathcal{M}^{\dagger}-i \not \partial \mathcal{M} \mathcal{M}^{\dagger}\right\rangle \psi_{L}-\frac{F^{-1}}{v^{2}} \bar{\psi}_{R} \mathcal{M}^{\dagger} U^{\dagger} \not L U \mathcal{M} \psi_{R}-\frac{1}{v^{2}} \bar{\psi}_{R} \mathcal{M}^{\prime \dagger} U^{\dagger} \not U \mathcal{M}^{\prime} \psi_{R} \\
& +\frac{\kappa}{v^{2}} F^{-1 / 2}\left(\bar{\psi}_{R} \mathcal{M}^{\dagger} U^{\dagger} \not U U \mathcal{M}^{\prime} \psi_{R}+\text { H.c. }\right)-\frac{\kappa}{v^{2}} F^{-1 / 2}\left(\bar{\psi}_{L} U \mathcal{M}^{\prime} \mathcal{M}^{\dagger} U^{\dagger} \not \psi_{L}+\text { H.c. }\right)
\end{aligned}
$$

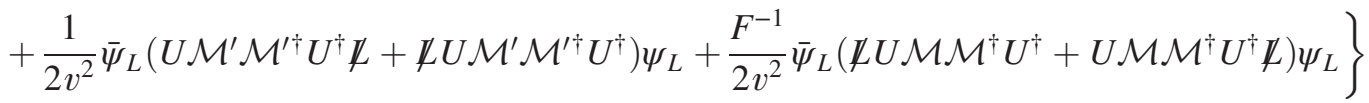

$$
\begin{aligned}
& +\Delta^{(1 / 2)} \mathcal{L}_{\psi^{2} U h D^{2}}+\Delta^{(1 / 2)} \mathcal{L}_{\psi^{4} U h}
\end{aligned}
$$

with

$$
\begin{aligned}
\Delta^{(1 / 2)} \mathcal{L}_{\psi^{2} U h D^{2}}= & -\frac{1}{16 \pi^{2}} \frac{1}{d-4}\left\{\left\langle L^{\mu} L_{\mu}\right\rangle\left[\frac{F \mathcal{B}}{2 v^{2}} \bar{\psi}_{L} U \mathcal{M}^{\prime \prime} \psi_{R}-\frac{\kappa^{2}-1}{F v^{2}} \bar{\psi}_{L} U\left(\frac{F^{\prime}}{2} \mathcal{M}^{\prime}-\mathcal{M}\right) \psi_{R}+\text { H.c. }\right]\right. \\
& \left.+\frac{2 \kappa^{\prime}}{v^{2}} \partial^{\mu} \eta\left(i \bar{\psi}_{L} L_{\mu} U\left(F^{-1 / 2} \mathcal{M}\right)^{\prime} \psi_{R}+\text { H.c. }\right)+\frac{3 \mathcal{B}}{F v^{2}} \partial^{\mu} \eta \partial_{\mu} \eta\left(\bar{\psi}_{L} U\left(\frac{F^{\prime}}{2} \mathcal{M}^{\prime}-\mathcal{M}\right) \psi_{R}+\text { H.c. }\right)\right\}, \\
\Delta^{(1 / 2)} \mathcal{L}_{\psi^{4} U h}= & -\frac{1}{16 \pi^{2}} \frac{1}{d-4}\left\{\frac{3 F^{-2}}{2 v^{4}}\left(\bar{\psi}_{L} U\left(\frac{F^{\prime}}{2} \mathcal{M}^{\prime}-\mathcal{M}\right) \psi_{R}+\text { H.c. }\right)^{2}+\frac{1}{2 v^{4}}\left(\bar{\psi}_{L} U \mathcal{M}^{\prime \prime} \psi_{R}+\text { H.c. }\right)^{2}\right. \\
& \left.+\frac{4}{v^{4}}\left(i \bar{\psi}_{L} U T^{a}\left(F^{-1 / 2} \mathcal{M}\right)^{\prime} \psi_{R}+\text { H.c. }\right)^{2}\right\} .
\end{aligned}
$$

The first five lines in the above expression of $\mathcal{L}_{\text {div }}^{(1 / 2)}$ correspond to structures already present in the lowest-order Lagrangian $\mathcal{L}_{2}$. The five lines that follow are of the type $\psi^{2} U h D$, but are not present in the basis considered in Ref. [14]. They need therefore to be transformed, proceeding as for the last term in $\Delta^{(0)} \mathcal{L}_{2}$. Considering the first term of this type, one may write (in these expressions and the following ones, only the terms between square brackets after the integration symbol depend on the variable of integration $s$ ) 


$$
\begin{aligned}
\frac{3 F^{-1}}{2 v^{2}} \bar{\psi}_{R}\left(\mathcal{M}^{\dagger} i \not \partial \mathcal{M}-i \not \partial \mathcal{M}^{\dagger} \mathcal{M}\right) \psi_{R}= & \frac{3}{2 v^{2}} \bar{\psi}_{R}\left[F^{-1 / 2} \mathcal{M}^{\dagger} i \not \partial\left(F^{-1 / 2} \mathcal{M}\right)-i \not \partial\left(F^{-1 / 2} \mathcal{M}^{\dagger}\right) F^{-1 / 2} \mathcal{M}\right] \psi_{R} \\
= & \frac{3}{2 v^{2}} i\left(\partial_{\mu} \eta\right) \bar{\psi}_{R} \gamma^{\mu}\left[F^{-1 / 2} \mathcal{M}^{\dagger}\left(F^{-1 / 2} \mathcal{M}\right)^{\prime}-\left(F^{-1 / 2} \mathcal{M}^{\dagger}\right)^{\prime} F^{-1 / 2} \mathcal{M}\right] \psi_{R} \\
= & \frac{3}{2 v^{2}} \bar{\psi}_{R} i \not \partial \int_{0}^{\eta} d s\left[F^{-1 / 2} \mathcal{M}^{\dagger}\left(F^{-1 / 2} \mathcal{M}\right)^{\prime}-\left(F^{-1 / 2} \mathcal{M}^{\dagger}\right)^{\prime} F^{-1 / 2} \mathcal{M}^{-1 / \psi_{R}}\right. \\
= & -\frac{3}{2 v^{2}} \bar{\psi}_{R} \int_{0}^{\eta} d s\left[F^{-1 / 2} \mathcal{M}^{\dagger}\left(F^{-1 / 2} \mathcal{M}\right)^{\prime}-\left(F^{-1 / 2} \mathcal{M}^{\dagger}\right)^{\prime} F^{-1 / 2} \mathcal{M}^{\prime} \mathcal{M}^{\dagger} U^{\dagger} \psi_{L}\right. \\
& +\frac{3}{2 v^{2}} \bar{\psi}_{L} U \mathcal{M} \int_{0}^{\eta} d s\left[F^{-1 / 2} \mathcal{M}^{\dagger}\left(F^{-1 / 2} \mathcal{M}\right)^{\prime}-\left(F^{-1 / 2} \mathcal{M}^{\dagger}\right)^{\prime} F^{-1 / 2} \mathcal{M}\right] \psi_{R} .
\end{aligned}
$$

In the last step, an integration by parts has been performed, the resulting total-derivative term has been dropped, and the lowest-order equations of motion for the fermionic fields have been used. The other structures of this type can be handled in a similar manner to obtain:

$$
\begin{aligned}
& \frac{1}{2 v^{2}} \bar{\psi}_{R}\left(\mathcal{M}^{\prime \dagger} i \not \partial \mathcal{M}^{\prime}-i \not \partial \mathcal{M}^{\prime \dagger} \mathcal{M}^{\prime}\right) \psi_{R}=\frac{1}{2 v^{2}} \bar{\psi}_{R} \int_{0}^{\eta} d s\left[\mathcal{M}^{\prime \prime \dagger} \mathcal{M}^{\prime}-\mathcal{M}^{\prime \dagger} \mathcal{M}^{\prime \prime}\right] \mathcal{M}^{\dagger} U^{\dagger} \psi_{L} \\
& +\frac{1}{2 v^{2}} \bar{\psi}_{L} U \mathcal{M} \int_{0}^{\eta} d s\left[\mathcal{M}^{\prime \dagger} \mathcal{M}^{\prime \prime}-\mathcal{M}^{\prime \prime \dagger} \mathcal{M}^{\prime}\right] \psi_{R} \\
& \frac{F^{-1}}{2 v^{2}} \bar{\psi}_{L} U\left(\mathcal{M} i \not \partial \mathcal{M}^{\dagger}-i \not \partial \mathcal{M} \mathcal{M}^{\dagger}\right) U^{\dagger} \psi_{L}=+\frac{1}{2 v^{2}} \bar{\psi}_{R} \mathcal{M}^{\dagger} \int_{0}^{\eta} d s\left[F^{-1 / 2} \mathcal{M}\left(F^{-1 / 2} \mathcal{M}^{\dagger}\right)^{\prime}-\left(F^{-1 / 2} \mathcal{M}\right)^{\prime} F^{-1 / 2} \mathcal{M}^{\dagger}\right] U^{\dagger} \psi_{L} \\
& +\frac{1}{2 v^{2}} \bar{\psi}_{L} U \int_{0}^{\eta} d s\left[\left(F^{-1 / 2} \mathcal{M}\right)^{\prime} F^{-1 / 2} \mathcal{M}^{\dagger}-F^{-1 / 2} \mathcal{M}\left(F^{-1 / 2} \mathcal{M}^{\dagger}\right)^{\prime}\right] \mathcal{M} \psi_{R} \\
& +\frac{1}{2 v^{2}} \bar{\psi}_{L} U \int_{0}^{\eta} d s\left[\left(F^{-1 / 2} \mathcal{M}\right)^{\prime} F^{-1 / 2} \mathcal{M}^{\dagger}-F^{-1 / 2} \mathcal{M}\left(F^{-1 / 2} \mathcal{M}^{\dagger}\right)^{\prime}\right] U^{\dagger} \not \psi_{L} \\
& +\frac{1}{2 v^{2}} \bar{\psi}_{L} \not L U \int_{0}^{\eta} d s\left[F^{-1 / 2} \mathcal{M}\left(F^{-1 / 2} \mathcal{M}^{\dagger}\right)^{\prime}-\left(F^{-1 / 2} \mathcal{M}\right)^{\prime} F^{-1 / 2} \mathcal{M}^{\dagger}\right] U^{\dagger} \psi_{L}, \\
& \frac{1}{2 v^{2}} \bar{\psi}_{L} U\left(\mathcal{M}^{\prime} i \not \partial \mathcal{M}^{\prime \dagger}-i \not \partial \mathcal{M}^{\prime} \mathcal{M}^{\prime \dagger}\right) U^{\dagger} \psi_{L}=+\frac{1}{2 v^{2}} \bar{\psi}_{R} \mathcal{M}^{\dagger} \int_{0}^{\eta} d s\left[\mathcal{M}^{\prime} \mathcal{M}^{\prime \prime \dagger}-\mathcal{M}^{\prime \prime} \mathcal{M}^{\prime \dagger}\right] U^{\dagger} \psi_{L} \\
& +\frac{1}{2 v^{2}} \bar{\psi}_{L} U \int_{0}^{\eta} d s\left[\mathcal{M}^{\prime \prime} \mathcal{M}^{\prime \dagger}-\mathcal{M}^{\prime} \mathcal{M}^{\prime \prime \dagger}\right] \mathcal{M} \psi_{R} \\
& +\frac{1}{2 v^{2}} \bar{\psi}_{L} U \int_{0}^{\eta} d s\left[\mathcal{M}^{\prime \prime} \mathcal{M}^{\prime \dagger}-\mathcal{M}^{\prime} \mathcal{M}^{\prime \prime \dagger}\right] U^{\dagger} \psi \psi_{L} \\
& +\frac{1}{2 v^{2}} \bar{\psi}_{L} \not L U \int_{0}^{\eta} d s\left[\mathcal{M}^{\prime} \mathcal{M}^{\prime \prime \dagger}-\mathcal{M}^{\prime \prime} \mathcal{M}^{\prime \dagger}\right] U^{\dagger} \psi_{L} \\
& \frac{F^{-1}}{v^{2}} \bar{\psi}_{L}\left\langle\mathcal{M} i \not \partial \mathcal{M}^{\dagger}-i \not \partial \mathcal{M} \mathcal{M}^{\dagger}\right\rangle \psi_{L}=-\frac{1}{v^{2}} \bar{\psi}_{R} \mathcal{M}^{\dagger} U^{\dagger} \int_{0}^{\eta} d s\left[\left\langle\left(F^{-1 / 2} \mathcal{M}\right)^{\prime} F^{-1 / 2} \mathcal{M}^{\dagger}-F^{-1 / 2} \mathcal{M}\left(F^{-1 / 2} \mathcal{M}^{\dagger}\right)^{\prime}\right\rangle\right] \psi_{L} \\
& -\frac{1}{v^{2}} \bar{\psi}_{L} \int_{0}^{\eta} d s\left[\left\langle F^{-1 / 2} \mathcal{M}\left(F^{-1 / 2} \mathcal{M}^{\dagger}\right)^{\prime}-\left(F^{-1 / 2} \mathcal{M}\right)^{\prime} F^{-1 / 2} \mathcal{M}^{\dagger}\right\rangle\right] U \mathcal{M} \psi_{R} \text {, }
\end{aligned}
$$

which reduce to structures already present in $\mathcal{L}_{2}$. The structures of the form $\psi_{I} O_{1} \not O_{2} \psi_{I}$, where $I=L, R$, can be handled upon using the identity (3.13), such that the whole structure can be expressed in terms of the basis of Ref. [14]. One thus obtains the decomposition

$$
\mathcal{L}_{\text {div }}^{(1 / 2)}=\Delta^{(1 / 2)} \mathcal{L}_{2}+\Delta^{(1 / 2)} \mathcal{L}_{\psi^{2} U h D}+\Delta^{(1 / 2)} \mathcal{L}_{\psi^{2} U h D^{2}}+\Delta^{(1 / 2)} \mathcal{L}_{\psi^{4} U h}
$$

with (the lowest-order equations of motion of the fermion fields have been applied) 


$$
\begin{aligned}
\Delta^{(1 / 2)} \mathcal{L}_{2}= & -\frac{1}{16 \pi^{2}} \frac{1}{d-4}\left\{\frac{3}{4} g^{2} \bar{\psi}_{L} U \mathcal{M} \psi_{R}+g^{\prime 2} \bar{\psi}_{L} Y_{L}^{2} U \mathcal{M} \psi_{R}+g^{\prime 2} \bar{\psi}_{L} U \mathcal{M} Y_{R}^{2} \psi_{R}-6 g_{s}^{2} C_{F} \bar{q} U \mathcal{M}_{q} P_{R} q+\frac{V^{\prime \prime}}{v^{4}} \bar{\psi}_{L} U \mathcal{M}^{\prime \prime} \psi_{R}\right. \\
& -8 g^{\prime 2} \bar{\psi}_{L} Y_{L} U \mathcal{M} Y_{R} \psi_{R}+\left(\left(3 g^{2}+g^{\prime 2}\right) \frac{v^{2}}{4} F+\frac{3}{2} \frac{F^{\prime} V^{\prime}}{F v^{2}}\right) \frac{F^{-1}}{v^{2}} \bar{\psi}_{L} U\left(\frac{F^{\prime}}{2} \mathcal{M}^{\prime}-\mathcal{M}\right) \psi_{R} \\
& +\frac{3}{v^{2}} F^{-1} \bar{\psi}_{L} U \mathcal{M} \mathcal{M}^{\dagger} \mathcal{M} \psi_{R}-\frac{3}{v^{2}} F^{-1} \bar{\psi}_{L} U\left\langle\mathcal{M} \mathcal{M}^{\dagger}\right\rangle \mathcal{M} \psi_{R} \\
& +\frac{2}{v^{2}} \bar{\psi}_{L} U \mathcal{M}^{\prime} \mathcal{M}^{\dagger} \mathcal{M}^{\prime} \psi_{R}+\frac{1}{2 v^{2}} \bar{\psi}_{L} U \mathcal{M} \mathcal{M}^{\prime \dagger} \mathcal{M}^{\prime} \psi_{R}+\frac{1}{2 v^{2}} \bar{\psi}_{L} U \mathcal{M}^{\prime} \mathcal{M}^{\prime \dagger} \mathcal{M} \psi_{R} \\
& +\frac{3}{2 v^{2}} \bar{\psi}_{L} U \mathcal{M} \int_{0}^{\eta} d s\left[F^{-1 / 2} \mathcal{M}^{\dagger}\left(F^{-1 / 2} \mathcal{M}\right)^{\prime}-\left(F^{-1 / 2} \mathcal{M}^{\dagger}\right)^{\prime} F^{-1 / 2} \mathcal{M}\right] \psi_{R} \\
& -\frac{1}{2 v^{2}} \bar{\psi}_{L} U \int_{0}^{\eta} d s\left[\left(F^{-1 / 2} \mathcal{M}\right)^{\prime} F^{-1 / 2} \mathcal{M}^{\dagger}-F^{-1 / 2} \mathcal{M}\left(F^{-1 / 2} \mathcal{M}^{\dagger}\right)^{\prime}\right] \mathcal{M} \psi_{R} \\
& +\frac{1}{2 v^{2}} \bar{\psi}_{L} U \mathcal{M} \int_{0}^{\eta} d s\left[\mathcal{M}^{\prime \dagger} \mathcal{M}^{\prime \prime}-\mathcal{M}^{\prime \prime \dagger} \mathcal{M}^{\prime}\right] \psi_{R}+\frac{1}{2 v^{2}} \bar{\psi}_{L} U \int_{0}^{\eta} d s\left[\mathcal{M}^{\prime \prime} \mathcal{M}^{\prime \dagger}-\mathcal{M}^{\prime} \mathcal{M}^{\prime \prime \dagger}\right] \mathcal{M} \psi_{R} \\
& \left.-\frac{1}{v^{2}} \bar{\psi}_{L} U \int_{0}^{\eta} d s\left[\left\langle F^{-1 / 2} \mathcal{M}\left(F^{-1 / 2} \mathcal{M}^{\dagger}\right)^{\prime}-\left(F^{-1 / 2} \mathcal{M}\right)^{\prime} F^{-1 / 2} \mathcal{M}^{\dagger}\right\rangle\right] \mathcal{M}_{R}+H_{. c .}\right\},
\end{aligned}
$$

and

$$
\begin{aligned}
\Delta^{(1 / 2)} \mathcal{L}_{\psi^{2} U h D}= & -\frac{1}{16 \pi^{2}} \frac{1}{d-4}\left\{-\frac{F^{-1}}{v^{2}} \bar{\psi}_{R} \mathcal{M}^{\dagger} U^{\dagger} \not U U \mathcal{M} \psi_{R}-\frac{1}{v^{2}} \bar{\psi}_{R} \mathcal{M}^{\prime \dagger} U^{\dagger} \mathcal{L} U^{\prime} \psi_{R}\right. \\
& +\frac{\kappa}{v^{2}} F^{-1 / 2}\left(\bar{\psi}_{R} \mathcal{M}^{\dagger} U^{\dagger} \not L \mathcal{M}^{\prime} \psi_{R}+\text { H.c. }\right)-\frac{\kappa}{v^{2}} F^{-1 / 2}\left(\bar{\psi}_{L} U \mathcal{M}^{\prime} \mathcal{M}^{\dagger} U^{\dagger} Z_{\psi_{L}}+\text { H.c. }\right) \\
& +\frac{1}{2 v^{2}}\left(\bar{\psi}_{L} U \mathcal{M}^{\prime} \mathcal{M}^{\prime \dagger} U^{\dagger} \not \psi_{L}+\text { H.c. }\right)+\frac{F^{-1}}{2 v^{2}}\left(\bar{\psi}_{L} U \mathcal{M} \mathcal{M}^{\dagger} U^{\dagger} \not \psi_{L}+\text { H.c. }\right) \\
& -\frac{1}{2 v^{2}}\left(\bar{\psi}_{L} U \int_{0}^{\eta} d s\left[\left(F^{-1 / 2} \mathcal{M}\right)^{\prime} F^{-1 / 2} \mathcal{M}^{\dagger}-F^{-1 / 2} \mathcal{M}\left(F^{-1 / 2} \mathcal{M}^{\dagger}\right)^{\prime}\right] U^{\dagger} \not \psi_{L}+\text { H.c. }\right) \\
& \left.+\frac{1}{2 v^{2}}\left(\bar{\psi}_{L} U \int_{0}^{\eta} d s\left[\mathcal{M}^{\prime \prime} \mathcal{M}^{\prime \dagger}-\mathcal{M}^{\prime} \mathcal{M}^{\prime \prime \dagger}\right] U^{\dagger} \mathcal{L}_{L}+\text { H.c. }\right)\right\} .
\end{aligned}
$$

\section{Summary}

In this section, the divergences at next-to-leading order have been decomposed into the basis of counterterms given in Ref. [14]. The result of this procedure is summarized by

$$
\begin{aligned}
\mathcal{L}_{\text {div }}= & {\left[\Delta^{(0)} \mathcal{L}_{2}+\Delta^{(1)} \mathcal{L}_{2}+\Delta^{(1 / 2)} \mathcal{L}_{2}\right]+\left[\Delta^{(0)} \mathcal{L}_{\beta_{1}}+\Delta^{(1)} \mathcal{L}_{\beta_{1}}\right]+\Delta^{(0)} \mathcal{L}_{U h D^{4}}+\left[\Delta^{(1)} \mathcal{L}_{\psi^{2} U h D}+\Delta^{(1 / 2)} \mathcal{L}_{\psi^{2} U h D}\right] } \\
& +\Delta^{(1 / 2)} \mathcal{L}_{\psi^{2} U h D^{2}}+\Delta^{(1 / 2)} \mathcal{L}_{\psi^{4} U h}
\end{aligned}
$$

where the expressions for each term are given above.

\section{RENORMALIZATION OF THE LEADING-ORDER LAGRANGIAN}

The renormalization of the parameters in $\mathcal{L}_{2}$ is derived from the first bracket in (3.31),

$$
\Delta \mathcal{L}_{2} \equiv \Delta^{(0)} \mathcal{L}_{2}+\Delta^{(1)} \mathcal{L}_{2}+\Delta^{(1 / 2)} \mathcal{L}_{2}
$$

The expressions of $\Delta^{(1)} \mathcal{L}_{2}, \Delta^{(0)} \mathcal{L}_{2}$, and $\Delta^{(1 / 2)} \mathcal{L}_{2}$ are given in Eqs. (3.10), (3.16), and (3.29), respectively. We rewrite (4.1) as

$$
\Delta \mathcal{L}_{2}=\Delta \mathcal{L}_{2, \text { gauge }}+\Delta \mathcal{L}_{2, \text { scalar }}
$$


where

$$
\begin{aligned}
32 \pi^{2} \varepsilon \Delta \mathcal{L}_{2, \text { gauge }}= & -\frac{g_{s}^{2}}{4} G^{\alpha \mu \nu} G_{\mu \nu}^{\alpha}\left(-\frac{22}{3} N_{c}+\frac{4}{3} N_{f}\right)-\frac{g^{2}}{2}\left\langle W^{\mu \nu} W_{\mu \nu}\right\rangle\left[-\frac{44}{3}+\frac{2}{3}\left(N_{c}+1\right) N_{\mathrm{g}}+\frac{1}{3}\right] \\
& -\frac{g^{\prime 2}}{4} B^{\mu \nu} B_{\mu \nu}\left[2\left(\frac{11}{27} N_{c}+1\right) N_{\mathrm{g}}+\frac{1}{3}\right] \\
& 32 \pi^{2} \varepsilon \Delta \mathcal{L}_{2, \text { scalar }}=\frac{v^{2}}{4}\left\langle L_{\mu} L^{\mu}\right\rangle A_{F}+\partial_{\mu} h \partial^{\mu} h A_{h}+A_{V}-\left(\bar{\psi} U A_{\mathcal{M}} P_{R} \psi+\text { H.c. }\right) .
\end{aligned}
$$

Here and in the following, we define for the dimension of space-time

$$
d \equiv 4-2 \varepsilon .
$$

The various functions introduced on the right-hand side of (4.4) read

$$
\begin{aligned}
& A_{F}(\eta)=-\frac{g^{\prime 2}}{2}\left(\kappa^{2}+3\right) F-\frac{g^{2}}{6}\left(19 \kappa^{2}+17\right) F-2\left(\kappa^{2}-1\right) \frac{F^{\prime} V^{\prime}}{F v^{4}}+2 \frac{V^{\prime \prime}}{v^{4}} \mathcal{B} F+\frac{4}{v^{2}}\langle\mathcal{M} \dagger \mathcal{M}\rangle, \\
& A_{h}(\eta)=\frac{1}{4}\left(3 g^{2}+g^{\prime 2}\right)\left(F \mathcal{B}-4 \kappa^{2}\right)+\frac{3}{2} \frac{F^{\prime} V^{\prime}}{F v^{4}} \mathcal{B}+\frac{2}{v^{2}}\left\langle\left\langle\mathcal{M}^{\prime \dagger} \mathcal{M}^{\prime}\right\rangle\right\rangle, \\
& A_{V}(\eta)= \\
& A_{\mathcal{M}}(\eta)=-\frac{3}{2}\left(3 g^{4}+2 g^{2} g^{\prime 2}+g^{\prime 4}\right) \frac{v^{4}}{16} F^{2}+\frac{3 g^{2}+g^{\prime 2}}{8} F^{\prime} V^{\prime}+\frac{3}{8}\left(\frac{F^{\prime} V^{\prime}}{F v^{2}}\right)^{2}+\frac{1}{2}\left(\frac{V^{\prime \prime}}{v^{2}}\right)^{2}-2\left\langle\left\langle\left(\mathcal{M}^{\dagger} \mathcal{M}\right)^{2}\right\rangle\right\rangle, \\
&- {\left[\left(3 g^{2}+g^{\prime 2}\right) \frac{v^{2}}{4} F+\frac{3}{2} \frac{F^{\prime} V^{\prime}}{F v^{2}}\right] \frac{F^{-1}}{v^{2}}\left(\frac{F^{\prime}}{2} \mathcal{M}^{\prime}-\mathcal{M}\right)-\frac{V^{\prime \prime}}{v^{4}} \mathcal{M}^{\prime \prime} } \\
&-\frac{3}{v^{2}} F^{-1} \mathcal{M} \mathcal{M}^{\dagger} \mathcal{M}+\frac{3}{v^{2}} F^{-1}\left\langle\mathcal{M} \mathcal{M}^{\dagger}\right\rangle \mathcal{M}-\frac{2}{v^{2}} \mathcal{M}^{\prime} \mathcal{M}^{\dagger} \mathcal{M}^{\prime}-\frac{1}{2 v^{2}} \mathcal{M} \mathcal{M}^{\prime \dagger} \mathcal{M}^{\prime}-\frac{1}{2 v^{2}} \mathcal{M}^{\prime} \mathcal{M}^{\prime \dagger} \mathcal{M} \\
&-\frac{3}{2 v^{2}} \mathcal{M} \int_{0}^{\eta} d s\left[F^{-1 / 2} \mathcal{M}^{\dagger}\left(F^{-1 / 2} \mathcal{M}\right)^{\prime}-\left(F^{-1 / 2} \mathcal{M}^{\dagger}\right)^{\prime} F^{-1 / 2} \mathcal{M}\right] \\
&+\frac{1}{2 v^{2}} \int_{0}^{\eta} d s\left[\left(F^{-1 / 2} \mathcal{M}\right)^{\prime} F^{-1 / 2} \mathcal{M}^{\dagger}-F^{-1 / 2} \mathcal{M}\left(F^{-1 / 2} \mathcal{M}^{\dagger}\right)^{\prime}\right] \mathcal{M} \\
&-\frac{1}{2 v^{2}} \mathcal{M} \int_{0}^{\eta} d s\left[\mathcal{M} \mathcal{M}^{\prime \dagger} \mathcal{M}^{\prime \prime}-\mathcal{M}^{\prime \prime \dagger} \mathcal{M}^{\prime}\right]-\frac{1}{2 v^{2}} \int_{0}^{\eta} d s\left[\mathcal{M}^{\prime \prime} \mathcal{M}{ }^{\prime \dagger}-\mathcal{M}^{\prime} \mathcal{M}^{\prime \prime \dagger}\right] \mathcal{M} \\
&+\frac{1}{v^{2}} \int_{0}^{\eta} d s\left[\left\langle F^{-1 / 2} \mathcal{M}\left(F^{-1 / 2} \mathcal{M}^{\dagger}\right)^{\prime}-\left(F^{-1 / 2} \mathcal{M}\right)^{\prime} F^{-1 / 2} \mathcal{M}^{\dagger}\right\rangle\right] \mathcal{M} \\
&\left.+\frac{8}{v^{2}} \mathcal{M} T^{3} \int_{0}^{\eta} d s\left\langle\left(F^{-1 / 2} \mathcal{M}^{\dagger}\right)^{\prime} F^{-1 / 2} \mathcal{M} T^{3}-F^{-1 / 2} \mathcal{M}^{\dagger}\left(F^{-1 / 2} \mathcal{M}\right)^{\prime} T^{3}\right\rangle\right\rangle .
\end{aligned}
$$

In the expression for $A_{\mathcal{M}}$ it is understood that the contribution proportional to $g_{s}^{2}$ only affects the quark fields.

The renormalization that cancels $\Delta \mathcal{L}_{2 \text {,gauge }}$ is standard and will be summarized below. In order to treat $\Delta \mathcal{L}_{2, \text { scalar }}$, it is convenient to first apply suitable redefinitions of the Higgs field $h$, which bring $\Delta \mathcal{L}_{2 \text {,scalar }}$ to a canonical form. The renormalization of $\mathcal{L}_{2}$ then proceeds in three steps. First, we eliminate the term $\partial_{\mu} h \partial^{\mu} h A_{h}$ from $\Delta \mathcal{L}_{2 \text {,scalar }}$ using integration by parts and the lowest-order equation of motion for $h$. Second, we shift the Higgs field, $\eta \rightarrow \eta+\delta$, and fix $\delta$ such that the minimum of the potential remains at $\eta=0$. In a third step, we renormalize the fields and parameters in $\mathcal{L}_{2}$. The resulting counterterms are then determined in the usual way from the requirement that they cancel the divergences of $\Delta \mathcal{L}_{2}$. 
We remark that for the field shift $\delta$, only the divergent part, relevant for the UV renormalization, is considered in the present section. However, $\delta$ also includes a finite piece, which must be chosen such as to preserve the condition $V^{\prime}(0)=0$. Minimal subtraction is therefore not sufficient here. The finite counterterm that is required in this case is computed in Sec. VIII.

\section{A. Redefinitions of the Higgs field}

In a first step, we eliminate $\partial_{\mu} h \partial^{\mu} h A_{h}$ from (4.4) by writing

$$
\begin{aligned}
\partial_{\mu} h \partial^{\mu} h A_{h}(\eta) & =-v \partial^{2} h \int_{0}^{\eta} d s A_{h}(s) \\
& =\left[-F^{\prime} \frac{v^{2}}{4}\left\langle L_{\mu} L^{\mu}\right\rangle+V^{\prime}+\bar{\psi} m^{\prime}(\eta, U) \psi\right] \int_{0}^{\eta} d s A_{h}(s),
\end{aligned}
$$

where we used an integration by parts and the equation of motion of the Higgs field (A4). Inserting (4.10) in (4.4) we obtain

$$
\begin{aligned}
32 \pi^{2} \varepsilon \Delta \mathcal{L}_{2, \text { scalar }}= & \frac{v^{2}}{4}\left\langle L_{\mu} L^{\mu}\right\rangle\left[A_{F}-F^{\prime} \int_{0}^{\eta} d s A_{h}(s)\right]+A_{V}+V^{\prime} \int_{0}^{\eta} d s A_{h}(s) \\
& -\left(\bar{\psi} U\left[A_{\mathcal{M}}-\mathcal{M}^{\prime} \int_{0}^{\eta} d s A_{h}(s)\right] P_{R} \psi+\text { H.c. }\right) .
\end{aligned}
$$

This step is equivalent to a field redefinition for $h$. The form of (4.11) has the advantage that the Higgs kinetic term $\partial h \partial h$ no longer receives a (divergent) one-loop correction. Consequently, there is no need to renormalize $h$. This is fully analogous to our treatment of the fermions, where we have also used the equations of motion to dispense with their field renormalization.

In a second step, we shift $\eta \rightarrow \eta+\delta$ to ensure a minimum of the potential at $\eta=0$. Since $V^{\prime}(0)=0$ at leading order, $\delta$ is of the order of a one-loop contribution.
The shift then leads to an additional one-loop term in the Lagrangian:

$$
\mathcal{L}_{2} \rightarrow \mathcal{L}_{2}+\frac{v^{2}}{4}\left\langle L_{\mu} L^{\mu}\right\rangle F^{\prime} \delta-V^{\prime} \delta-\left(\bar{\psi} U \mathcal{M}^{\prime} \delta P_{R} \psi+\text { H.c. }\right) .
$$

The divergent Lagrangian $\Delta \mathcal{L}_{2, \text { scalar }}$ then becomes

$$
\begin{aligned}
32 \pi^{2} \varepsilon \Delta \mathcal{L}_{2, \text { scalar }}= & \frac{v^{2}}{4}\left\langle L_{\mu} L^{\mu}\right\rangle\left[A_{F}-F^{\prime} \int_{0}^{\eta} d s A_{h}(s)+F^{\prime} \tilde{\delta}\right]+A_{V}+V^{\prime} \int_{0}^{\eta} d s A_{h}(s)-V^{\prime} \tilde{\delta} \\
& -\left(\bar{\psi} U\left[A_{\mathcal{M}}-\mathcal{M}^{\prime} \int_{0}^{\eta} d s A_{h}(s)+\mathcal{M}^{\prime} \tilde{\delta}\right] P_{R} \psi+\text { H.c. }\right),
\end{aligned}
$$

where

$$
\tilde{\delta} \equiv 32 \pi^{2} \varepsilon \delta
$$

The condition that the minimum of the effective potential remains at $\eta=0$ implies for the divergent part of $\delta$

$$
\left[\left(A_{V}+V^{\prime} \int_{0}^{\eta} d s A_{h}(s)\right)^{\prime}-V^{\prime \prime} \tilde{\delta}\right]_{\eta=0}=0
$$

and accordingly

$$
\begin{aligned}
\tilde{\delta}= & \frac{3 F_{1}}{32 V_{2}}\left(3 g^{4}+2 g^{2} g^{\prime 2}+g^{4}\right)+\frac{3 g^{2}+g^{\prime 2}}{8} F_{1}+6 V_{3} \\
& -\frac{2}{v^{4} V_{2}}\left\langle\left\langle\left(\mathcal{M}_{1}^{\dagger} \mathcal{M}_{0}+\mathcal{M}_{0}^{\dagger} \mathcal{M}_{1}\right) \mathcal{M}_{0}^{\dagger} \mathcal{M}_{0}\right\rangle\right\rangle .
\end{aligned}
$$

\section{B. Renormalization of $\mathcal{L}_{\mathbf{2}}$}

We start from the leading-order Lagrangian (2.1), where we consider all fields and parameters $X$ as unrenormalized, denoted by $\stackrel{\circ}{X}$. As discussed above, no renormalization is 
needed for the Higgs field and the fermions, and we thus take $\stackrel{\circ}{h}=h, \stackrel{\circ}{\psi}=\psi$. For the remaining quantities we introduce renormalization constants in the form

$$
\begin{array}{lll}
\stackrel{\circ}{G}_{\mu}^{\alpha}=Z_{G}^{1 / 2} G_{\mu}^{\alpha}, & \stackrel{\circ}{W}_{\mu}^{a}=Z_{W}^{1 / 2} W_{\mu}^{a}, & \stackrel{\circ}{B}_{\mu}=Z_{B}^{1 / 2} B_{\mu}, \\
\stackrel{\circ}{g}_{s}=Z_{G}^{-1 / 2} g_{s} \mu^{\varepsilon}, \quad \stackrel{\circ}{g}=Z_{W}^{-1 / 2} g \mu^{\varepsilon}, & \stackrel{\circ}{g}=Z_{B}^{-1 / 2} g^{\prime} \mu^{\varepsilon}, \\
\stackrel{\circ}{\varphi}=Z_{\varphi}^{1 / 2} \varphi^{a}, & \stackrel{\circ}{v}=Z_{\varphi}^{1 / 2} v \mu^{-\varepsilon}, & \\
\stackrel{\circ}{F}_{n}=Z_{F_{n}} F_{n}, \quad \stackrel{\circ}{V_{n}}=Z_{V_{n}} V_{n} \mu^{2 \varepsilon}, & \stackrel{\circ}{\mathcal{M}_{n}}=\mathcal{M}_{n}+\Delta \mathcal{M}_{n} .
\end{array}
$$

Notice that the renormalization of the gauge fields and of the corresponding gauge couplings involve the same renormalization constants. This is because we are using the background field gauge, with the effect that the Slavnov-Taylor identities boil down to the simple QEDtype Ward-Takahashi identities [23].

Since

$$
\frac{v^{2}}{4}\left\langle L_{\mu} L^{\mu}\right\rangle=\frac{1}{2} \partial_{\mu} \varphi^{a} \partial^{\mu} \varphi^{a}+\frac{v^{2}}{8}\left(g W_{\mu}^{a}-g^{\prime} B_{\mu} \delta^{a 3}\right)^{2}+\cdots,
$$

we observe that the Goldstone kinetic term and the gaugeboson mass term receive the same divergent one-loop corrections. In addition, the term $g W_{\mu}^{a}-g^{\prime} B_{\mu} \delta^{a 3}$ is not renormalized within our scheme. It follows that the renormalization factors for $\varphi^{a}$ and $v$ are identical, a fact which we have already used in (4.17). As a consequence, $\varphi^{a} / v$ and the Goldstone matrix $U$ are not renormalized.

It will be convenient to write the one-loop $Z$-factor for a quantity $X$ in the minimal subtraction (MS) scheme as

$$
Z_{X}=1+\Delta Z_{X}=1+\frac{\Delta \tilde{Z}_{X}}{32 \pi^{2} \varepsilon}, \quad \Delta \tilde{Z}_{X} \equiv 32 \pi^{2} \varepsilon \Delta Z_{X},
$$

$\Delta \tilde{\mathcal{M}}_{n} \equiv 32 \pi^{2} \varepsilon \Delta \mathcal{M}_{n}$

In the $\overline{\mathrm{MS}}$ scheme, the pole term is replaced by

$$
\frac{1}{\varepsilon} \equiv \frac{2}{4-d} \rightarrow \frac{2}{4-d}-\gamma_{E}+\ln 4 \pi
$$

Employing (4.17) in the unrenormalized version of (2.1), and subtracting the renormalized Lagrangian, we obtain the counterterm $\mathcal{L}_{2, C T}=\stackrel{\circ}{\mathcal{L}}_{2}-\mathcal{L}_{2}$, where

$$
\begin{aligned}
\mathcal{L}_{2, C T}= & -\frac{1}{4}\left(Z_{G}-1\right) G_{\mu \nu}^{\alpha} G^{\alpha \mu \nu}-\frac{1}{2}\left(Z_{W}-1\right)\left\langle W_{\mu \nu} W^{\mu \nu}\right\rangle-\frac{1}{4}\left(Z_{B}-1\right) B_{\mu \nu} B^{\mu \nu} \\
& +\frac{v^{2}}{4}\left\langle L_{\mu} L^{\mu}\right\rangle \sum_{n=0}^{\infty}\left(Z_{\varphi}^{1-n / 2} Z_{F_{n}}-1\right) F_{n} \eta^{n}-\sum_{n=2}^{\infty}\left(Z_{\varphi}^{2-n / 2} Z_{V_{n}}-1\right) v^{4} V_{n} \eta^{n} \\
& -\left(\bar{\psi} U \sum_{n=0}^{\infty}\left(Z_{\varphi}^{-n / 2}\left(\mathcal{M}_{n}+\Delta \mathcal{M}_{n}\right)-\mathcal{M}_{n}\right) \eta^{n} P_{R} \psi+\text { H.c. }\right) \\
= & -\frac{1}{4} \Delta Z_{G} G_{\mu \nu}^{\alpha} G^{\alpha \mu \nu}-\frac{1}{2} \Delta Z_{W}\left\langle W_{\mu \nu} W^{\mu \nu}\right\rangle-\frac{1}{4} \Delta Z_{B} B_{\mu \nu} B^{\mu \nu} \\
& +\frac{v^{2}}{4}\left\langle L_{\mu} L^{\mu}\right\rangle \sum_{n=0}^{\infty}\left(\left(1-\frac{n}{2}\right) \Delta Z_{\varphi}+\Delta Z_{F_{n}}\right) F_{n} \eta^{n}-v^{4} \sum_{n=2}^{\infty}\left(\left(2-\frac{n}{2}\right) \Delta Z_{\varphi}+\Delta Z_{V_{n}}\right) V_{n} \eta^{n} \\
& -\left(\bar{\psi} U \sum_{n=0}^{\infty}\left(-\frac{n}{2} \Delta Z_{\varphi} \mathcal{M}_{n}+\Delta \mathcal{M}_{n}\right) \eta^{n} P_{R} \psi+\text { H.c. }\right) .
\end{aligned}
$$

The approximation in the second equality holds to one-loop order.

The renormalization constants are fixed by requiring $-\mathcal{L}_{2, C T}=\Delta \mathcal{L}_{2 \text {,gauge }}+\Delta \mathcal{L}_{2, \text { scalar }}$, with $\Delta \mathcal{L}_{2 \text {,gauge }}$ in (4.3) and $\Delta \mathcal{L}_{2, \text { scalar }}$ in the redefined form of (4.13). This implies

$$
\begin{aligned}
& \Delta \tilde{Z}_{G}=-\left(-\frac{22}{3} N_{c}+\frac{4}{3} N_{f}\right) g_{s}^{2}, \\
& \Delta \tilde{Z}_{W}=-\left[-\frac{44}{3}+\frac{2}{3}\left(N_{c}+1\right) N_{\mathrm{g}}+\frac{1}{3}\right] g^{2}, \\
& \Delta \tilde{Z}_{B}=-\left[2\left(\frac{11}{27} N_{c}+1\right) N_{\mathrm{g}}+\frac{1}{3}\right] g^{\prime 2}
\end{aligned}
$$


and

$$
\begin{gathered}
\sum_{n=0}^{\infty}\left(\left(1-\frac{n}{2}\right) \Delta \tilde{Z}_{\varphi}+\Delta \tilde{Z}_{F_{n}}\right) F_{n} \eta^{n} \\
=-A_{F}+F^{\prime} \int_{0}^{\eta} d s A_{h}(s)-F^{\prime} \tilde{\delta}, \\
\sum_{n=2}^{\infty}\left(\left(2-\frac{n}{2}\right) \Delta \tilde{Z}_{\varphi}+\Delta \tilde{Z}_{V_{n}}\right) v^{4} V_{n} \eta^{n} \\
=A_{V}+V^{\prime} \int_{0}^{\eta} d s A_{h}(s)-V^{\prime} \tilde{\delta}, \\
\sum_{n=0}^{\infty}\left(-\frac{n}{2} \Delta \tilde{Z}_{\varphi} \mathcal{M}_{n}+\Delta \tilde{\mathcal{M}}_{n}\right) \eta^{n} \\
=-A_{\mathcal{M}}+\mathcal{M}^{\prime} \int_{0}^{\eta} d s A_{h}(s)-\mathcal{M}^{\prime} \tilde{\delta} .
\end{gathered}
$$

$\Delta \tilde{Z}_{\varphi}$ follows from the $n=0$ term in (4.23), noting that $F_{0} \equiv 1$ and $\Delta \tilde{Z}_{F_{0}} \equiv 0$. We find

$$
\begin{aligned}
\Delta \tilde{Z}_{\varphi}= & \left(\frac{F_{1}^{2}}{4} \frac{5}{3}+\frac{17}{6}\right) g^{2}+\frac{3}{2} g^{\prime 2}-V_{2} F_{1}^{2}+4 V_{2} F_{2}-6 F_{1} V_{3} \\
& -\frac{4}{v^{2}}\left\langle\left\langle\mathcal{M}_{0}^{\dagger} \mathcal{M}_{0}\right\rangle\right\rangle-\frac{3 F_{1}^{2}}{32 V_{2}}\left(3 g^{4}+2 g^{2} g^{\prime 2}+g^{\prime 4}\right) \\
& +\frac{2 F_{1}}{v^{4} V_{2}}\left\langle\left\langle\left(\mathcal{M}_{1}^{\dagger} \mathcal{M}_{0}+\mathcal{M}_{0}^{\dagger} \mathcal{M}_{1}\right) \mathcal{M}_{0}^{\dagger} \mathcal{M}_{0}\right\rangle\right\rangle .
\end{aligned}
$$

Equations (4.22)-(4.25), together with (4.26), fix the complete set of one-loop counterterms that renormalize the parameters contained in the leading-order Lagrangian (2.1).

\section{Renormalization group equations for the coefficients of $\mathcal{L}_{2}$}

Knowing the renormalization constants, it is straightforward to derive the renormalization-group beta functions. For any parameter $X$ we define

$$
\beta_{X}=16 \pi^{2} \frac{d X}{d \ln \mu}
$$

Recalling (4.19), we have

$$
\stackrel{\circ}{X}=Z_{X} X \mu^{r \varepsilon} \Rightarrow \beta_{X}=-\frac{1}{2 \varepsilon} \frac{d \Delta \tilde{Z}_{X}}{d \ln \mu} X=\Delta \tilde{Z}_{X} X .
$$

In the last step, we have used

$$
\frac{d \Delta \tilde{Z}_{X}}{d \ln \mu}=-2 \varepsilon \Delta \tilde{Z}_{X}
$$

which holds for $X=g_{s}, g, g^{\prime}, v^{2}, F_{n}, V_{n}$, and also for $\Delta \tilde{Z}_{X} \rightarrow \Delta \tilde{\mathcal{M}}_{n}$. In all these cases, $\Delta \tilde{Z}_{X}$ is a homogeneous function of degree 2 in the weak couplings $k=g_{s}, g, g^{\prime}$, $\sqrt{V_{n}}, \mathcal{M}_{n} / v$. This implies (4.29), since at tree level in $d=4-2 \varepsilon$ dimensions

$$
\frac{d k}{d \ln \mu}=-\varepsilon k, \quad \text { whereas } \frac{d F_{n}}{d \ln \mu}=\frac{d \mathcal{M}_{n}}{d \ln \mu}=0 .
$$

Using (4.28) and the results of Sec. IV B, we finally obtain

$$
\begin{gathered}
\beta_{g_{s}}=-\frac{1}{2} \Delta \tilde{Z}_{G} g_{s}, \quad \beta_{g}=-\frac{1}{2} \Delta \tilde{Z}_{W} g, \quad \beta_{g^{\prime}}=-\frac{1}{2} \Delta \tilde{Z}_{B} g^{\prime} \\
\sum_{n=0}^{\infty} \beta_{F_{n}} \eta^{n}=-A_{F}+F^{\prime} \int_{0}^{\eta} d s A_{h}(s)-F^{\prime} \tilde{\delta} \\
-\left(F-\frac{\eta}{2} F^{\prime}\right) \Delta \tilde{Z}_{\varphi}, \\
\quad-\left(2 V-\frac{\eta}{2} V^{\prime}\right) \Delta \tilde{Z}_{\varphi}, \\
v^{4} \sum_{n=2}^{\infty} \beta_{V_{n}} \eta^{n}=A_{V}+V^{\prime} \int_{0}^{\eta} d s A_{h}(s)-V^{\prime} \tilde{\delta} \\
\sum_{n=0}^{\infty} \beta_{\mathcal{M}_{n}} \eta^{n}=-A_{\mathcal{M}}+\mathcal{M}^{\prime} \int_{0}^{\eta} d s A_{h}(s)-\mathcal{M}^{\prime} \tilde{\delta}+\frac{\eta}{2} \mathcal{M}^{\prime} \Delta \tilde{Z}_{\varphi},
\end{gathered}
$$

$$
\beta_{v^{2}}=\Delta \tilde{Z}_{\varphi} v^{2} .
$$

The functions $A_{F}, A_{h}, A_{V}$, and $A_{\mathcal{M}}$ are defined in (4.6)-(4.9).

Equations (4.31)-(4.35) summarize the one-loop beta functions in the MS (or $\overline{\mathrm{MS}}$ ) scheme for all the parameters of the leading-order electroweak chiral Lagrangian (2.1). We emphasize that the beta functions for the gauge couplings (4.31) are identical with their SM expressions. Adopting NLO bases different from ours may change the definition of the gauge couplings, and hence their beta functions, by a redistribution of NLO terms. We have checked that (4.32)-(4.35) are in agreement with the SM results, when the corresponding limit is taken. In particular, the function on the right-hand side of (4.32) vanishes in this limit, as it should.

\section{Running of the $h V V$ coupling $F_{1}$}

As an example for the RGE running of anomalous Higgs couplings within the EWChL we consider $F_{1}$, the coupling of $h$ to a pair of vector bosons. From (4.32) we find 


$$
\begin{aligned}
\beta_{F_{1}}= & \frac{3}{64 V_{2}} F_{1}\left(F_{1}^{2}-4 F_{2}\right)\left(3 g^{4}+2 g^{2} g^{\prime 2}+g^{\prime 4}\right)-\frac{g^{2}}{12} F_{1}\left[\frac{37}{4}\left(F_{1}^{2}-4 F_{2}\right)+17\left(F_{2}-1\right)\right]-\frac{3}{16} g^{\prime 2} F_{1}\left(F_{1}^{2}-4\right) \\
& +V_{2}\left[F_{1}\left(\frac{5}{2}\left(F_{1}^{2}-4 F_{2}\right)+4\left(F_{2}-1\right)\right)+12 F_{3}\right]-\frac{F_{1}^{2}-4 F_{2}}{v^{4} V_{2}}\left\langle\left\langle\mathcal{M}_{0}^{\dagger} \mathcal{M}_{0}\left(\mathcal{M}_{0}^{\dagger} \mathcal{M}_{1}+\mathcal{M}_{1}^{\dagger} \mathcal{M}_{0}\right)\right\rangle\right\rangle \\
& +2 \frac{F_{1}-2}{v^{2}}\left\langle\left\langle\mathcal{M}_{0}^{\dagger} \mathcal{M}_{0}+\mathcal{M}_{1}^{\dagger} \mathcal{M}_{1}\right\rangle\right\rangle+\frac{4}{v^{2}}\left\langle\left\langle\left(\mathcal{M}_{1}^{\dagger}-\mathcal{M}_{0}^{\dagger}\right)\left(\mathcal{M}_{1}-\mathcal{M}_{0}\right)\right\rangle\right\rangle
\end{aligned}
$$

In the limit of large top mass and Yukawa coupling this becomes

$$
\beta_{F_{1}} \approx\left(4 F_{2}-F_{1}^{2}\right) 4 N_{c} \frac{m_{t}^{4}}{v^{2} m_{h}^{2}}
$$

Therefore

$$
\begin{aligned}
\frac{F_{1}(\mu)}{2} & \approx \frac{F_{1}(v)}{2}+\frac{\beta_{F_{1}}}{32 \pi^{2}} \ln \frac{\mu}{v} \\
& \approx \frac{F_{1}(v)}{2}+0.036\left(4 F_{2}-F_{1}^{2}\right) \ln \frac{\mu}{v} \\
& \approx \frac{F_{1}(v)}{2}+0.125\left(4 F_{2}-F_{1}^{2}\right),
\end{aligned}
$$

where in the last expression we have taken $\mu=8 \mathrm{TeV}$ as a representative cutoff scale for the EWChL. Experimentally $F_{1} / 2$ is close to 1 (within $10 \%$ ), but $F_{2}$ may still deviate significantly from its $\mathrm{SM}$ value $F_{2}=1$. Equation (4.38) indicates that the difference between $F_{1}$ at the electroweak scale $v$ and at the high scale $\mu$ may be appreciable.

\section{RENORMALIZATION OF THE NEXT-TO-LEADING-ORDER COUNTERTERMS}

Since $\mathcal{L}_{2}$ is not renormalizable, one also needs to introduce a set $\mathcal{L}_{4}$ of new operators in order to absorb all the divergences generated at one loop. The structure of $\mathcal{L}_{4}$ is entirely dictated by the symmetries of $\mathcal{L}_{2}$ and by power counting. A complete set of counterterms at the oneloop level is already available [14] and we will stick to the operator basis displayed there.

In the previous section we dealt with the divergences that can be absorbed through renormalization of the leadingorder Lagrangian $\mathcal{L}_{2}$. The remaining divergences have to be eliminated through the renormalization of the counterterms at next-to-leading order. These remaining divergences are given by

$$
\begin{aligned}
\mathcal{L}_{\text {div }}-\Delta \mathcal{L}_{2}= & \Delta \mathcal{L}_{\beta_{1}}+\Delta \mathcal{L}_{U h D^{4}}+\Delta \mathcal{L}_{\psi^{2} U h D} \\
& +\Delta \mathcal{L}_{\psi^{2} U h D^{2}}+\Delta \mathcal{L}_{\psi^{4} U h},
\end{aligned}
$$

with

$$
\begin{aligned}
& \Delta \mathcal{L}_{\beta_{1}} \equiv \Delta^{(0)} \mathcal{L}_{\beta_{1}}+\Delta^{(1)} \mathcal{L}_{\beta_{1}}=-\frac{1}{16 \pi^{2}} \frac{1}{d-4}\left(-\frac{5}{6}\right) g^{\prime 2} v^{2}\left(\kappa^{2}-1\right) F\left\langle\tau_{L} L^{\mu}\right\rangle\left\langle\tau_{L} L_{\mu}\right\rangle \\
& \Delta \mathcal{L}_{U h D^{4}} \equiv \Delta^{(0)} \mathcal{L}_{U h D^{4}}=-\frac{1}{16 \pi^{2}} \frac{1}{d-4}\left\{\left(\frac{\left(\kappa^{2}-1\right)^{2}}{12}+\frac{F^{2} \mathcal{B}^{2}}{8}\right)\left\langle L^{\mu} L_{\mu}\right\rangle^{2}+\frac{\left(\kappa^{2}-1\right)^{2}}{6}\left\langle L_{\mu} L_{\nu}\right\rangle\left\langle L^{\mu} L^{\nu}\right\rangle\right. \\
& \left.-\left(\left(\kappa^{2}-1\right) \mathcal{B}+\frac{\kappa^{\prime 2}}{6}\right)\left\langle L^{\mu} L_{\mu}\right\rangle \partial^{\nu} \eta \partial_{\nu} \eta+\frac{2}{3} \kappa^{\prime 2}\left\langle L_{\mu} L_{\nu}\right\rangle \partial^{\mu} \eta \partial^{\nu} \eta+\frac{3}{2} \mathcal{B}^{2}\left(\partial^{\mu} \eta \partial_{\mu} \eta\right)^{2}\right\} \\
& \Delta \mathcal{L}_{\psi^{2} U h D} \equiv \Delta^{(1 / 2)} \mathcal{L}_{\psi^{2} U h D}+\Delta^{(1)} \mathcal{L}_{\psi^{2} U h D} \\
& =-\frac{1}{16 \pi^{2}} \frac{1}{d-4}\left\{\bar{\psi}_{L} U \boldsymbol{Q}\left(\mathcal{M}, \mathcal{M}^{\dagger}\right) U^{\dagger} \mathcal{L}_{L}+\bar{\psi}_{L} \not L U \mathfrak{Q}\left(\mathcal{M}, \mathcal{M}^{\dagger}\right)^{\dagger} U^{\dagger} \psi_{L}\right. \\
& +\frac{\kappa^{2}-1}{12}\left[2 g^{\prime 2}\left\langle\tau_{L} L^{\nu}\right\rangle \bar{e}_{R} \gamma_{\nu} e_{R}-2 g^{\prime 2}\left\langle\tau_{L} L^{\nu}\right\rangle \bar{\psi}_{L} \gamma_{\nu} Y_{L} \psi_{L}-\frac{4}{3} g^{\prime 2}\left\langle\tau_{L} L^{\nu}\right\rangle \bar{u}_{R} \gamma_{\nu} u_{R}+\frac{2}{3} g^{\prime 2}\left\langle\tau_{L} L^{\nu}\right\rangle \bar{d}_{R} \gamma_{\nu} d_{R}\right] \\
& \left.-\frac{F^{-1}}{v^{2}} \bar{\psi}_{R} \mathcal{M}^{\dagger} U^{\dagger} \not L U \mathcal{M} \psi_{R}-\frac{1}{v^{2}} \bar{\psi}_{R} \mathcal{M}^{\prime \dagger} U^{\dagger} \not L U \mathcal{M}^{\prime} \psi_{R}+\frac{\kappa}{v^{2}} F^{-1 / 2}\left(\bar{\psi}_{R} \mathcal{M}^{\dagger} U^{\dagger} \not L U^{\prime} \psi_{R}+\text { H.c. }\right)\right\},
\end{aligned}
$$




$$
\begin{aligned}
\Delta \mathcal{L}_{\psi^{2} U h D^{2}} \equiv & \Delta^{(1 / 2)} \mathcal{L}_{\psi^{2} U h D^{2}} \\
= & -\frac{1}{16 \pi^{2}} \frac{1}{d-4}\left\{\left\langle L^{\mu} L_{\mu}\right\rangle\left[\frac{F \mathcal{B}}{2 v^{2}} \bar{\psi}_{L} U \mathcal{M}^{\prime \prime} \psi_{R}-\frac{\kappa^{2}-1}{F v^{2}} \bar{\psi}_{L} U\left(\frac{F^{\prime}}{2} \mathcal{M}^{\prime}-\mathcal{M}\right) \psi_{R}+\text { H.c. }\right]\right. \\
& \left.+\frac{2 \kappa^{\prime}}{v^{2}} \partial^{\mu} \eta\left(i \bar{\psi}_{L} L_{\mu} U\left(F^{-1 / 2} \mathcal{M}\right)^{\prime} \psi_{R}+\text { H.c. }\right)+\frac{3 \mathcal{B}}{F v^{2}} \partial^{\mu} \eta \partial_{\mu} \eta\left(\bar{\psi}_{L} U\left(\frac{F^{\prime}}{2} \mathcal{M}^{\prime}-\mathcal{M}\right) \psi_{R}+\text { H.c. }\right)\right\}, \\
\Delta \mathcal{L}_{\psi^{4} U h} \equiv & \Delta^{(1 / 2)} \mathcal{L}_{\psi^{4} U h} \\
= & -\frac{1}{16 \pi^{2}} \frac{1}{d-4}\left\{\frac{3 F^{-2}}{2 v^{4}}\left(\bar{\psi}_{L} U\left(\frac{F^{\prime}}{2} \mathcal{M}^{\prime}-\mathcal{M}\right) \psi_{R}+\text { H.c. }\right)^{2}+\frac{1}{2 v^{4}}\left(\bar{\psi}_{L} U \mathcal{M}^{\prime \prime} \psi_{R}+\text { H.c. }\right)^{2}\right. \\
& \left.+\frac{4}{v^{4}}\left(i \bar{\psi}_{L} U T^{a}\left(F^{-1 / 2} \mathcal{M}\right)^{\prime} \psi_{R}+\text { H.c. }\right)^{2}\right\} .
\end{aligned}
$$

In the expression of $\Delta \mathcal{L}_{\psi^{2} U h D}$, the quantity

$$
\begin{aligned}
\mathfrak{L}\left(\mathcal{M}, \mathcal{M}^{\dagger}\right)= & g^{2} \frac{\kappa^{2}-1}{24}+\frac{1}{2 v^{2}}\left[\mathcal{M}^{\prime} \mathcal{M}^{\prime \dagger}+F^{-1} \mathcal{M} \mathcal{M}^{\dagger}\right]-\frac{\kappa}{v^{2}} F^{-1 / 2} \mathcal{M}^{\prime} \mathcal{M}^{\dagger} \\
& -\frac{1}{2 v^{2}} \int_{0}^{\eta} d s\left[\mathcal{M}^{\prime} \mathcal{M}^{\prime \prime \dagger}-\mathcal{M}^{\prime \prime} \mathcal{M}^{\prime \dagger}-F^{-1} \mathcal{M} \mathcal{M}^{\dagger \prime}+F^{-1} \mathcal{M}^{\prime} \mathcal{M}^{\dagger}\right]
\end{aligned}
$$

has been introduced. Notice that $\mathfrak{Q}\left(\mathcal{M}_{\nu}, \mathcal{M}_{\nu}^{\dagger}\right)=g^{2}\left(\kappa^{2}-1\right) / 24$.

\section{A. Renormalization of the counterterm $\beta_{1}$}

Let us start with the elimination of the divergent term $\Delta \mathcal{L}_{\beta_{1}}$. It requires the counterterm $\mathcal{L}_{\beta_{1}}$ given in [14], namely (note the slight change in notation as compared to this reference)

$$
\begin{aligned}
\mathcal{L}_{\beta_{1}} & =-v^{2}\left\langle\tau_{L} L^{\mu}\right\rangle\left\langle\tau_{L} L_{\mu}\right\rangle\left[\beta_{1}+F_{\beta_{1}}(\eta)\right], \\
F_{\beta_{1}}(\eta) & =\sum_{n \geq 1} f_{\beta_{1}, n} \eta^{n} .
\end{aligned}
$$

In order to perform the renormalization (for instance, in the $\overline{\mathrm{MS}}$ scheme), one interprets the coefficients as unrenormalized ones and writes

$$
\begin{gathered}
{\stackrel{\circ}{f_{\beta_{1}, n}}}=f_{\beta_{1}, n}(\mu)+\frac{\gamma_{\beta_{1}, n}}{16 \pi^{2}} \mu^{d-4}\left[\frac{1}{d-4}-\frac{1}{2}\left(\ln 4 \pi-\gamma_{E}\right)\right], \\
\circ{ }^{\circ}=\beta_{1}(\mu)+\frac{\gamma_{\beta_{1}}}{16 \pi^{2}} \mu^{d-4}\left[\frac{1}{d-4}-\frac{1}{2}\left(\ln 4 \pi-\gamma_{E}\right)\right],
\end{gathered}
$$

i.e.,

$$
\stackrel{\circ}{F}_{\beta_{1}}(\eta)=F_{\beta_{1}}(\eta ; \mu)+\frac{\Gamma_{\beta_{1}}(\eta)}{16 \pi^{2}} \mu^{d-4}\left[\frac{1}{d-4}-\frac{1}{2}\left(\ln 4 \pi-\gamma_{E}\right)\right],
$$

with

$$
\begin{aligned}
\gamma_{\beta_{1}} & =\frac{5}{24} g^{2}\left(F_{1}^{2}-4\right), \\
\Gamma_{\beta_{1}}(\eta) & =\frac{5}{6} g^{\prime 2}\left[\bar{\kappa}\left(\bar{\kappa}+F_{1}\right)+\bar{F}\left(\kappa^{2}-1\right)\right] .
\end{aligned}
$$

\section{B. Renormalization of the counterterms in the class $U h D^{4}$}

The elimination of the divergences contained in $\Delta^{(0)} \mathcal{L}_{U h D^{4}}$ requires five counterterms of the class $U h D^{4}$,

$$
\mathcal{L}_{U h D^{4}}=\sum_{i=1}^{15} \mathcal{O}_{D i}\left[c_{D i}+F_{D i}(\eta)\right], \quad F_{D i}(\eta)=\sum_{n \geq 1} f_{D i, n} \eta^{n}
$$

namely $\mathcal{O}_{D i}$ for $i=1,2,7,8,11$. Notice that in contrast to Ref. [14], we have not written the overall factor $v^{2} / \Lambda^{2}$, and we have introduced the specific couplings $c_{D i}$ in a different way. The renormalization proceeds as previously,

$$
\begin{gathered}
{\stackrel{\circ}{c_{D i}}}=c_{D i}(\mu)+\frac{\gamma_{D i}}{16 \pi^{2}} \mu^{d-4}\left[\frac{1}{d-4}-\frac{1}{2}\left(\ln 4 \pi-\gamma_{E}\right)\right], \\
\stackrel{\circ}{F}_{D i}(\eta)=F_{D i}(\eta ; \mu)+\frac{\Gamma_{D i}(\eta)}{16 \pi^{2}} \mu^{d-4}\left[\frac{1}{d-4}-\frac{1}{2}\left(\ln 4 \pi-\gamma_{E}\right)\right],
\end{gathered}
$$


with

$$
\begin{array}{ll}
\gamma_{D 1}=\frac{1}{12}\left(\frac{F_{1}^{2}}{4}-1\right)^{2}+\frac{1}{8}\left(\frac{F_{1}^{2}}{4}-F_{2}\right)^{2}, & \gamma_{D 2}=\frac{1}{6}\left(\frac{F_{1}^{2}}{4}-1\right)^{2}, \\
\gamma_{D 7}=-\frac{1}{6}\left(\frac{F_{1}^{2}}{4}-F_{2}\right)\left(\frac{7}{4} F_{1}^{2}-F_{2}-6\right), & \gamma_{D 8}=\frac{2}{3}\left(\frac{F_{1}^{2}}{4}-F_{2}\right)^{2}, \quad \gamma_{D 11}=\frac{3}{2}\left(\frac{F_{1}^{2}}{4}-F_{2}\right)^{2},
\end{array}
$$

and

$$
\begin{aligned}
\Gamma_{D 1} & =\frac{1}{12} \bar{\kappa}\left(\bar{\kappa}+F_{1}\right)\left[\bar{\kappa}\left(\bar{\kappa}+F_{1}\right)+\frac{F_{1}^{2}}{2}-2\right]+\frac{1}{8}\left(F \mathcal{B}-\frac{F_{1}^{2}}{4}+F_{2}\right)\left(F \mathcal{B}+\frac{F_{1}^{2}}{4}-F_{2}\right), \\
\Gamma_{D 2} & =\frac{1}{6} \bar{\kappa}\left(\bar{\kappa}+F_{1}\right)\left[\bar{\kappa}\left(\bar{\kappa}+F_{1}\right)+\frac{F_{1}^{2}}{2}-2\right] \\
\Gamma_{D 7} & =-\overline{\mathcal{B}}\left(\frac{F_{1}^{2}}{4}-1\right)-\mathcal{B} \bar{\kappa}\left(\bar{\kappa}+F_{1}\right)-\frac{\bar{F}}{6}\left(\frac{F_{1}^{2}}{4}-F_{2}\right)^{2}-\frac{F}{6} \overline{\mathcal{B}}\left(\overline{\mathcal{B}}+\frac{F_{1}^{2}}{2}-2 F_{2}\right), \\
\Gamma_{D 8} & =\frac{2}{3} F \overline{\mathcal{B}}\left(\overline{\mathcal{B}}+\frac{F_{1}^{2}}{2}-2 F_{2}\right)+\frac{2}{3} \bar{F}\left(\frac{F_{1}^{2}}{4}-F_{2}\right)^{2}, \\
\Gamma_{D 11} & =\frac{3}{2} \overline{\mathcal{B}}\left(\overline{\mathcal{B}}+\frac{F_{1}^{2}}{2}-2 F_{2}\right) .
\end{aligned}
$$

\section{Renormalization of the counterterms in the class $\psi^{2} U h D$}

In order to proceed with the renormalization of the operators of the class $\psi^{2} U h D$, we first need to express $\mathcal{L}_{\psi^{2} U h D}$ in terms of the basis operators given in Ref. [14]. Recalling that $\mathcal{M}$ and therefore also $\mathfrak{L}$ of (5.7) are diagonal in $S U(2)$ space, one has

$$
\mathfrak{L}=\frac{1}{2}\langle\mathfrak{Q}\rangle+2 T^{3}\left\langle T^{3} \mathfrak{Q}\right\rangle,
$$

so that

$$
\bar{\psi}_{L} U \mathfrak{Q} U^{\dagger} \not L \psi_{L}=\frac{1}{2} \bar{\psi}_{L}\langle\mathfrak{Q}\rangle \not L \psi_{L}+2 \bar{\psi}_{L}\left\langle\mathfrak{R} T^{3}\right\rangle \tau_{L} \not \psi_{L} .
$$

Note that traces are over $S U(2)$ only, i.e., the expressions on the right-hand side of (5.16) are still matrices in flavor space.

Upon using the identity (3.13), (5.17) becomes $\left(P_{ \pm}=\frac{1}{2} \pm T^{3}, P_{12}=T^{1}+i T^{2}, P_{21}=T^{1}-i T^{2}\right)$

$$
\begin{aligned}
\bar{\psi}_{L} U \mathfrak{Q} U^{\dagger} \not \psi_{L}= & \bar{\psi}_{L} \gamma^{\mu}\langle\mathfrak{Q}\rangle \tau_{L} \psi_{L}\left\langle\tau_{L} L_{\mu}\right\rangle+\frac{1}{2} \bar{\psi}_{L} \gamma^{\mu}\langle\mathfrak{Q}\rangle U P_{12} U^{\dagger} \psi_{L}\left\langle U P_{21} U^{\dagger} L_{\mu}\right\rangle+\frac{1}{2} \bar{\psi}_{L} \gamma^{\mu}\langle\mathfrak{Q}\rangle U P_{21} U^{\dagger} \psi_{L}\left\langle U P_{12} U^{\dagger} L_{\mu}\right\rangle \\
& +\bar{\psi}_{L} \gamma^{\mu}\left\langle\mathfrak{Q} T^{3}\right\rangle \psi_{L}\left\langle\tau_{L} L_{\mu}\right\rangle+\bar{\psi}_{L} \gamma^{\mu}\left\langle\mathfrak{Q} T^{3}\right\rangle U P_{12} U^{\dagger} \psi_{L}\left\langle U P_{21} U^{\dagger} L_{\mu}\right\rangle-\bar{\psi}_{L} \gamma^{\mu}\left\langle\mathfrak{R} T^{3}\right\rangle U P_{21} U^{\dagger} \psi_{L}\left\langle U P_{12} U^{\dagger} L_{\mu}\right\rangle \\
= & \bar{\psi}_{L} \gamma^{\mu}\left\langle\mathfrak{Q} T^{3}\right\rangle \psi_{L}\left\langle\tau_{L} L_{\mu}\right\rangle+\bar{\psi}_{L} \gamma^{\mu}\langle\mathfrak{Q}\rangle \tau_{L} \psi_{L}\left\langle\tau_{L} L_{\mu}\right\rangle \\
& +\bar{\psi}_{L} \gamma^{\mu}\left\langle\mathfrak{Q} P_{+}\right\rangle U P_{12} U^{\dagger} \psi_{L}\left\langle U P_{21} U^{\dagger} L_{\mu}\right\rangle+\bar{\psi}_{L} \gamma^{\mu}\left\langle\mathfrak{Q} P_{-}\right\rangle U P_{21} U^{\dagger} \psi_{L}\left\langle U P_{12} U^{\dagger} L_{\mu}\right\rangle,
\end{aligned}
$$

due to the relations $T^{3} P_{12}=+P_{12} / 2$ and $T^{3} P_{21}=-P_{21} / 2$.

The terms involving the right-handed fermion fields can essentially be handled along similar lines. One first establishes an identity similar to (3.13),

$$
\begin{aligned}
\bar{\psi}_{R} O_{1} U^{\dagger} \not U U O_{2} \psi_{R} & =2 \bar{\psi}_{R} \gamma^{\mu} O_{1} T^{a} O_{2} \psi_{R}\left\langle U T^{a} U^{\dagger} L_{\mu}\right\rangle \\
& =\bar{\psi}_{R} \gamma^{\mu} O_{1} P_{12} O_{2} \psi_{R}\left\langle U P_{21} U^{\dagger} L_{\mu}\right\rangle+\bar{\psi}_{R} \gamma^{\mu} O_{1} P_{21} O_{2} \psi_{R}\left\langle U P_{12} U^{\dagger} L_{\mu}\right\rangle+2 \bar{\psi}_{R} \gamma^{\mu} O_{1} T^{3} O_{2} \psi_{R}\left\langle\tau_{L} L_{\mu}\right\rangle .
\end{aligned}
$$

Then one obtains, for instance, 


$$
\begin{aligned}
\bar{\psi}_{R} \mathcal{M}^{\dagger} U^{\dagger} \not U \mathcal{M} \psi_{R}= & 2 \bar{\psi}_{R} \gamma^{\mu} \mathcal{M}^{\dagger} T^{3} \mathcal{M} \psi_{R}\left\langle\tau_{L} L_{\mu}\right\rangle+\bar{\psi}_{R} \gamma^{\mu} \mathcal{M}^{\dagger} P_{12} \mathcal{M} \psi_{R}\left\langle U P_{21} U^{\dagger} L_{\mu}\right\rangle+\bar{\psi}_{R} \gamma^{\mu} \mathcal{M}^{\dagger} P_{21} \mathcal{M} \psi_{R}\left\langle U P_{12} U^{\dagger} L_{\mu}\right\rangle \\
= & \bar{u}_{R} \gamma^{\mu} \mathcal{M}_{u}^{\dagger} \mathcal{M}_{u} u_{R}\left\langle\tau_{L} L_{\mu}\right\rangle-\bar{d}_{R} \gamma^{\mu} \mathcal{M}_{d}^{\dagger} \mathcal{M}_{d} d_{R}\left\langle\tau_{L} L_{\mu}\right\rangle-\bar{e}_{R} \gamma^{\mu} \mathcal{M}_{e}^{\dagger} \mathcal{M}_{e} e_{R}\left\langle\tau_{L} L_{\mu}\right\rangle \\
& +\bar{u}_{R} \gamma^{\mu} \mathcal{M}_{u}^{\dagger} \mathcal{M}_{d} d_{R}\left\langle U P_{21} U^{\dagger} L_{\mu}\right\rangle+\bar{d}_{R} \gamma^{\mu} \mathcal{M}_{d}^{\dagger} \mathcal{M}_{u} u_{R}\left\langle U P_{12} U^{\dagger} L_{\mu}\right\rangle .
\end{aligned}
$$

These divergences can now be removed through the renormalization of the operators $\mathcal{O}_{\psi V i}$ (and $\mathcal{O}_{\psi V i}^{\dagger}$ ) of $\mathcal{L}_{\psi^{2} U h D}$. Explicitly, one has (notice that the functions $F_{\psi V i}$ are actually matrices in generation space)

$$
\stackrel{\circ}{F}_{\psi V i}(\eta)=F_{\psi V i}(\eta ; \mu)+\frac{\Gamma_{\psi V i}(\eta)}{16 \pi^{2}} \mu^{d-4}\left[\frac{1}{d-4}-\frac{1}{2}\left(\ln 4 \pi-\gamma_{E}\right)\right],
$$

with

$$
\begin{aligned}
& \Gamma_{\psi V 1}=-\frac{1}{2}\left(\mathfrak{\Omega}_{u}-\mathfrak{\Omega}_{d}+\text { H.c. }\right)+g^{\prime 2} \frac{\kappa^{2}-1}{36}, \\
& \Gamma_{\psi V 2}=-\mathfrak{Q}_{u}-\mathfrak{Q}_{d}+\text { H.c., } \\
& \Gamma_{\psi V 3}=-\mathfrak{Q}_{u}-\mathbf{Q}_{d}^{\dagger}, \\
& \Gamma_{\psi V 4}=+\frac{1}{v^{2}}\left[F^{-1} \mathcal{M}_{u}^{\dagger} \mathcal{M}_{u}+\mathcal{M}_{u}^{\prime \dagger} \mathcal{M}_{u}^{\prime}-\kappa F^{-1 / 2}\left(\mathcal{M}_{u}^{\dagger} \mathcal{M}_{u}^{\prime}+\mathcal{M}_{u}^{\prime \dagger} \mathcal{M}_{u}\right)\right]+g^{\prime 2} \frac{\kappa^{2}-1}{9}, \\
& \Gamma_{\psi V 5}=-\frac{1}{v^{2}}\left[F^{-1} \mathcal{M}_{d}^{\dagger} \mathcal{M}_{d}+\mathcal{M}_{d}^{\prime \dagger} \mathcal{M}_{d}^{\prime}-\kappa F^{-1 / 2}\left(\mathcal{M}_{d}^{\dagger} \mathcal{M}_{d}^{\prime}+\mathcal{M}_{d}^{\prime \dagger} \mathcal{M}_{d}\right)\right]-g^{\prime 2} \frac{\kappa^{2}-1}{18}, \\
& \Gamma_{\psi V 6}=+\frac{1}{v^{2}}\left[F^{-1} \mathcal{M}_{u}^{\dagger} \mathcal{M}_{d}+\mathcal{M}_{u}^{\prime \dagger} \mathcal{M}_{d}^{\prime}-\kappa F^{-1 / 2}\left(\mathcal{M}_{u}^{\dagger} \mathcal{M}_{d}^{\prime}+\mathcal{M}_{u}^{\prime \dagger} \mathcal{M}_{d}\right)\right] \text {, } \\
& \Gamma_{\psi V 7}=-\frac{1}{2}\left(\boldsymbol{\Omega}_{\nu}-\mathbf{\Omega}_{e}+\text { H.c. }\right)-g^{\prime 2} \frac{\kappa^{2}-1}{12}, \\
& \Gamma_{\psi V 8}=-\mathfrak{Q}_{\nu}-\mathfrak{Q}_{e}+\text { H.c., } \\
& \Gamma_{\psi V 9}=-\mathfrak{Q}_{\nu}-\mathfrak{Q}_{e}^{\dagger}, \\
& \Gamma_{\psi V 10}=-\frac{1}{v^{2}}\left[F^{-1} \mathcal{M}_{e}^{\dagger} \mathcal{M}_{e}+\mathcal{M}_{e}^{\prime \dagger} \mathcal{M}_{e}^{\prime}-\kappa F^{-1 / 2}\left(\mathcal{M}_{e}^{\dagger} \mathcal{M}_{e}^{\prime}+\mathcal{M}_{e}^{\prime \dagger} \mathcal{M}_{e}\right)\right]-g^{\prime 2} \frac{\kappa^{2}-1}{6} .
\end{aligned}
$$

In these expressions, $\mathfrak{Q}_{j} \equiv \mathfrak{L}\left(\mathcal{M}_{j}, \mathcal{M}_{j}^{\dagger}\right)$ with $j=u, d, \nu, e$.

\section{Renormalization of the counterterms in the class $\psi^{2} U h D^{2}$}

Recalling that the matrix $\mathcal{M}$ is $S U(2)$ diagonal, one has the following relations:

$$
\bar{\psi}_{L} U \mathcal{F}(\mathcal{M}) \psi_{R}=\bar{q}_{L} U P_{+} \mathcal{F}\left(\mathcal{M}_{u}\right) q_{R}+\bar{q}_{L} U P_{-} \mathcal{F}\left(\mathcal{M}_{d}\right) q_{R}+\bar{l}_{L} U P_{-} \mathcal{F}\left(\mathcal{M}_{e}\right) l_{R}
$$

$$
\begin{aligned}
\bar{\psi}_{L} L_{\mu} U \mathcal{F}(\mathcal{M}) \psi_{R}= & 2 \bar{\psi}_{L} U T^{a} \mathcal{F}(\mathcal{M}) \psi_{R}\left\langle U T^{a} U^{\dagger} L_{\mu}\right\rangle \\
= & \bar{\psi}_{L} U P_{12} \mathcal{F}(\mathcal{M}) \psi_{R}\left\langle U P_{21} U^{\dagger} L_{\mu}\right\rangle+\bar{\psi}_{L} U P_{21} \mathcal{F}(\mathcal{M}) \psi_{R}\left\langle U P_{12} U^{\dagger} L_{\mu}\right\rangle+2 \bar{\psi}_{L} U T^{3} \mathcal{F}(\mathcal{M}) \psi_{R}\left\langle\tau_{L} L_{\mu}\right\rangle \\
= & \bar{\psi}_{L} U P_{12} \mathcal{F}(\mathcal{M}) \psi_{R}\left\langle U P_{21} U^{\dagger} L_{\mu}\right\rangle+\bar{\psi}_{L} U P_{21} \mathcal{F}(\mathcal{M}) \psi_{R}\left\langle U P_{12} U^{\dagger} L_{\mu}\right\rangle \\
& +\bar{\psi}_{L} U P_{+} \mathcal{F}(\mathcal{M}) \psi_{R}\left\langle\tau_{L} L_{\mu}\right\rangle-\bar{\psi}_{L} U P_{-} \mathcal{F}(\mathcal{M}) \psi_{R}\left\langle\tau_{L} L_{\mu}\right\rangle \\
= & \bar{q}_{L} U P_{12} \mathcal{F}\left(\mathcal{M}_{d}\right) q_{R}\left\langle U P_{21} U^{\dagger} L_{\mu}\right\rangle+\bar{q}_{L} U P_{21} \mathcal{F}\left(\mathcal{M}_{u}\right) q_{R}\left\langle U P_{12} U^{\dagger} L_{\mu}\right\rangle+\bar{l}_{L} U P_{12} \mathcal{F}\left(\mathcal{M}_{e}\right) l_{R}\left\langle U P_{21} U^{\dagger} L_{\mu}\right\rangle \\
& +\bar{q}_{L} U P_{+} \mathcal{F}\left(\mathcal{M}_{u}\right) q_{R}\left\langle\tau_{L} L_{\mu}\right\rangle-\bar{q}_{L} U P_{-} \mathcal{F}\left(\mathcal{M}_{d}\right) q_{R}\left\langle\tau_{L} L_{\mu}\right\rangle-\bar{l}_{L} U P_{-} \mathcal{F}\left(\mathcal{M}_{e}\right) l_{R}\left\langle\tau_{L} L_{\mu}\right\rangle .
\end{aligned}
$$

Using them one can rewrite $\Delta^{(1 / 2)} \mathcal{L}_{\psi^{2} U h D^{2}}$ as 


$$
\begin{aligned}
\Delta^{(1 / 2)} \mathcal{L}_{\psi^{2} U h D^{2}}= & -\frac{1}{16 \pi^{2}} \frac{1}{d-4}\left\{\bar{q}_{L} U P_{+}\left[\frac{F \mathcal{B}}{2 v^{2}} \mathcal{M}_{u}^{\prime \prime}-\frac{\kappa^{2}-1}{F v^{2}}\left(\frac{F^{\prime}}{2} \mathcal{M}_{u}^{\prime}-\mathcal{M}_{u}\right)\right] q_{R}\left\langle L^{\mu} L_{\mu}\right\rangle\right. \\
& +\bar{q}_{L} U P_{-}\left[\frac{F \mathcal{B}}{2 v^{2}} \mathcal{M}_{d}^{\prime \prime}-\frac{\kappa^{2}-1}{F v^{2}}\left(\frac{F^{\prime}}{2} \mathcal{M}_{d}^{\prime}-\mathcal{M}_{d}\right)\right] q_{R}\left\langle L^{\mu} L_{\mu}\right\rangle \\
& +\bar{l}_{L} U P_{-}\left[\frac{F \mathcal{B}}{2 v^{2}} \mathcal{M}_{e}^{\prime \prime}-\frac{\kappa^{2}-1}{F v^{2}}\left(\frac{F^{\prime}}{2} \mathcal{M}_{e}^{\prime}-\mathcal{M}_{e}\right)\right] l_{R}\left\langle L^{\mu} L_{\mu}\right\rangle \\
& +\frac{2 i \kappa^{\prime}}{v^{2}} \partial^{\mu} \eta\left[\bar{q}_{L} U P_{12}\left(F^{-1 / 2} \mathcal{M}_{d}\right)^{\prime} q_{R}\left\langle U P_{21} U^{\dagger} L_{\mu}\right\rangle+\bar{q}_{L} U P_{21}\left(F^{-1 / 2} \mathcal{M}_{u}\right)^{\prime} q_{R}\left\langle U P_{12} U^{\dagger} L_{\mu}\right\rangle\right. \\
& +\bar{l}_{L} U P_{12}\left(F^{-1 / 2} \mathcal{M}_{e}\right)^{\prime} l_{R}\left\langle U P_{21} U^{\dagger} L_{\mu}\right\rangle+\bar{q}_{L} U P_{+}\left(F^{-1 / 2} \mathcal{M}_{u}\right)^{\prime} q_{R}\left\langle\tau_{L} L_{\mu}\right\rangle \\
& \left.-\bar{q}_{L} U P_{-}\left(F^{-1 / 2} \mathcal{M}_{d}\right)^{\prime} q_{R}\left\langle\tau_{L} L_{\mu}\right\rangle-\bar{l}_{L} U P_{-}\left(F^{-1 / 2} \mathcal{M}_{e}\right)^{\prime} l_{R}\left\langle\tau_{L} L_{\mu}\right\rangle\right] \\
& +\frac{3 \mathcal{B}}{F v^{2}} \partial^{\mu} \eta \partial_{\mu} \eta\left[\bar{q}_{L} U P_{+}\left(\frac{F^{\prime}}{2} \mathcal{M}_{u}^{\prime}-\mathcal{M}_{u}\right) q_{R}+\bar{q}_{L} U P_{-}\left(\frac{F^{\prime}}{2} \mathcal{M}_{d}^{\prime}-\mathcal{M}_{d}\right) q_{R}\right. \\
& \left.\left.+\bar{l}_{L} U P_{-}\left(\frac{F^{\prime}}{2} \mathcal{M}_{e}^{\prime}-\mathcal{M}_{e}\right) l_{R}\right]+ \text { H.c. }\right\} .
\end{aligned}
$$

These divergences are removed through the renormalization of the operators $\mathcal{O}_{\psi S i}$ (and $\mathcal{O}_{\psi S i}^{\dagger}$ ) of $\mathcal{L}_{\psi^{2} U h D^{2}}$, with (in order of appearance in the previous formula) $i=1,2,7,12,13,17,10,11,16,14,15,18$. Explicitly, one has (notice that the functions $F_{\psi S i}$ are actually matrices in generation space)

$$
\stackrel{\circ}{F}_{\psi S i}(\eta)=F_{\psi S i}(\eta ; \mu)+\frac{\Gamma_{\psi S i}(\eta)}{16 \pi^{2}} \mu^{d-4}\left[\frac{1}{d-4}-\frac{1}{2}\left(\ln 4 \pi-\gamma_{E}\right)\right],
$$

with

$$
\begin{aligned}
\Gamma_{\psi S 1} & =\frac{F \mathcal{B}}{2 v^{2}} \mathcal{M}_{u}^{\prime \prime}-\frac{\kappa^{2}-1}{F v^{2}}\left(\frac{F^{\prime}}{2} \mathcal{M}_{u}^{\prime}-\mathcal{M}_{u}\right), \quad \Gamma_{\psi S 2}=\frac{F \mathcal{B}}{2 v^{2}} \mathcal{M}_{d}^{\prime \prime}-\frac{\kappa^{2}-1}{F v^{2}}\left(\frac{F^{\prime}}{2} \mathcal{M}_{d}^{\prime}-\mathcal{M}_{d}\right), \\
\Gamma_{\psi S 7} & =\frac{F \mathcal{B}}{2 v^{2}} \mathcal{M}_{e}^{\prime \prime}-\frac{\kappa^{2}-1}{F v^{2}}\left(\frac{F^{\prime}}{2} \mathcal{M}_{e}^{\prime}-\mathcal{M}_{e}\right), \quad \Gamma_{\psi S 10}=\frac{2 i \kappa^{\prime}}{v^{2}}\left(F^{-1 / 2} \mathcal{M}_{u}\right)^{\prime}, \\
\Gamma_{\psi S 11} & =-\frac{2 i \kappa^{\prime}}{v^{2}}\left(F^{-1 / 2} \mathcal{M}_{d}\right)^{\prime}, \quad \Gamma_{\psi S 12}=\frac{2 i \kappa^{\prime}}{v^{2}}\left(F^{-1 / 2} \mathcal{M}_{d}\right)^{\prime}, \\
\Gamma_{\psi S 13} & =\frac{2 i \kappa^{\prime}}{v^{2}}\left(F^{-1 / 2} \mathcal{M}_{u}\right)^{\prime}, \quad \Gamma_{\psi S 14}=\frac{3 \mathcal{B}}{F v^{2}}\left(\frac{F^{\prime}}{2} \mathcal{M}_{u}^{\prime}-\mathcal{M}_{u}\right), \\
\Gamma_{\psi S 15} & =\frac{3 \mathcal{B}}{F v^{2}}\left(\frac{F^{\prime}}{2} \mathcal{M}_{d}^{\prime}-\mathcal{M}_{d}\right), \quad \Gamma_{\psi S 16}=-\frac{2 i \kappa^{\prime}}{v^{2}}\left(F^{-1 / 2} \mathcal{M}_{e}\right)^{\prime}, \\
\Gamma_{\psi S 17} & =\frac{2 i \kappa^{\prime}}{v^{2}}\left(F^{-1 / 2} \mathcal{M}_{e}\right)^{\prime}, \quad \Gamma_{\psi S 18}=\frac{3 \mathcal{B}}{F v^{2}}\left(\frac{F^{\prime}}{2} \mathcal{M}_{e}^{\prime}-\mathcal{M}_{e}\right) .
\end{aligned}
$$

\section{E. Renormalization of the counterterms in the class $\psi^{4} U h$}

The expression to start with reads

$$
\begin{aligned}
\Delta \mathcal{L}_{\psi^{4} U h}= & -\frac{1}{16 \pi^{2}} \frac{1}{d-4}\left\{\frac{3 F^{-2}}{2 v^{4}}\left(\bar{\psi}_{L} U\left(\frac{F^{\prime}}{2} \mathcal{M}^{\prime}-\mathcal{M}\right) \psi_{R}+\text { H.c. }\right)^{2}+\frac{1}{2 v^{4}}\left(\bar{\psi}_{L} U \mathcal{M}^{\prime \prime} \psi_{R}+\text { H.c. }\right)^{2}\right. \\
& \left.+\frac{4}{v^{4}}\left(i \bar{\psi}_{L} U T^{a}\left(F^{-1 / 2} \mathcal{M}\right)^{\prime} \psi_{R}+\text { H.c. }\right)^{2}\right\} \\
= & -\frac{1}{16 \pi^{2}} \frac{1}{d-4}\left\{\frac{3 F^{-2}}{2 v^{4}}\left(\bar{\psi}_{L} U\left(\frac{F^{\prime}}{2} \mathcal{M}^{\prime}-\mathcal{M}\right) \psi_{R}\right)^{2}+\frac{3 F^{-2}}{2 v^{4}}\left(\bar{\psi}_{R}\left(\frac{F^{\prime}}{2} \mathcal{M}^{\prime}-\mathcal{M}\right)^{\dagger} U^{\dagger} \psi_{L}\right)^{2}\right.
\end{aligned}
$$




$$
\begin{aligned}
& +\frac{3 F^{-2}}{v^{4}}\left(\bar{\psi}_{L} U\left(\frac{F^{\prime}}{2} \mathcal{M}^{\prime}-\mathcal{M}\right) \psi_{R}\right)\left(\bar{\psi}_{R}\left(\frac{F^{\prime}}{2} \mathcal{M}^{\prime}-\mathcal{M}\right)^{\dagger} U^{\dagger} \psi_{L}\right) \\
& +\frac{1}{2 v^{4}}\left(\bar{\psi}_{L} U \mathcal{M}^{\prime \prime} \psi_{R}\right)^{2}+\frac{1}{2 v^{4}}\left(\bar{\psi}_{R} \mathcal{M}^{\prime \prime \dagger} U^{\dagger} \psi_{L}\right)^{2}+\frac{1}{v^{4}}\left(\bar{\psi}_{L} U \mathcal{M}^{\prime \prime} \psi_{R}\right)\left(\bar{\psi}_{R} \mathcal{M}^{\prime \prime \dagger} U^{\dagger} \psi_{L}\right) \\
& -\frac{4}{v^{4}}\left(\bar{\psi}_{L} U T^{a}\left(F^{-1 / 2} \mathcal{M}\right)^{\prime} \psi_{R}\right)\left(\bar{\psi}_{L} U T^{a}\left(F^{-1 / 2} \mathcal{M}\right)^{\prime} \psi_{R}\right) \\
& -\frac{4}{v^{4}}\left(\bar{\psi}_{R}\left(F^{-1 / 2} \mathcal{M}^{\dagger}\right)^{\prime} T^{a} U^{\dagger} \psi_{L}\right)\left(\bar{\psi}_{R}\left(F^{-1 / 2} \mathcal{M}^{\dagger}\right)^{\prime} T^{a} U^{\dagger} \psi_{L}\right) \\
& \left.+\frac{8}{v^{4}}\left(\bar{\psi}_{L} U T^{a}\left(F^{-1 / 2} \mathcal{M}\right)^{\prime} \psi_{R}\right)\left(\bar{\psi}_{R}\left(F^{-1 / 2} \mathcal{M}^{\dagger}\right)^{\prime} T^{a} U^{\dagger} \psi_{L}\right)\right\} .
\end{aligned}
$$

We have to consider the following structures:

$$
\left(\bar{\psi}_{L} U \mathcal{F}(\mathcal{M}) \psi_{R}\right)^{2}+\text { H.c., } \quad\left(\bar{\psi}_{L} U \mathcal{F}(\mathcal{M}) \psi_{R}\right)\left(\bar{\psi}_{R} \mathcal{F}(\mathcal{M})^{\dagger} U^{\dagger} \psi_{L}\right)
$$

and

$$
\left(\bar{\psi}_{L} U T^{a} \mathcal{F}(\mathcal{M}) \psi_{R}\right)\left(\bar{\psi}_{L} U T^{a} \mathcal{F}(\mathcal{M}) \psi_{R}\right)+\text { H.c. }, \quad\left(\bar{\psi}_{L} U T^{a} \mathcal{F}(\mathcal{M}) \psi_{R}\right)\left(\bar{\psi}_{R} \mathcal{F}(\mathcal{M})^{\dagger} T^{a} U^{\dagger} \psi_{L}\right),
$$

and decompose them onto the operator basis of Ref. [14]. Notice that the coefficients of the four-fermion operators are actually rank-four tensors in generation space. We use the notation

$$
\begin{aligned}
\mathcal{F}^{(1)} \otimes \mathcal{F}^{(2)} \mathcal{O} & \equiv \mathcal{F}_{i j}^{(1)} \mathcal{F}_{k l}^{(2)} \bar{\psi}_{i} \ldots \psi_{j} \bar{\psi}_{k} \ldots \psi_{l}, \\
\mathcal{F}^{(1)} \tilde{\otimes} \mathcal{F}^{(2)} \mathcal{O} & \equiv \mathcal{F}_{i l}^{(1)} \mathcal{F}_{k j}^{(2)} \bar{\psi}_{i} \ldots \psi_{j} \bar{\psi}_{k} \ldots \psi_{l}
\end{aligned}
$$

for an operator $\mathcal{O} \equiv \bar{\psi}_{i} \ldots \psi_{j} \bar{\psi}_{k} \ldots \psi_{l}$, where $i, j, k, l$ are generation indices. For the basis operators $\mathcal{O}_{A B}$ to be used below, we follow the notation of [24].

Using Eq. (5.23), one obtains

$$
\begin{aligned}
\left(\bar{\psi}_{L} U \mathcal{F}(\mathcal{M}) \psi_{R}\right)^{2}= & \left(\bar{q}_{L} U P_{+} \mathcal{F}\left(\mathcal{M}_{u}\right) q_{R}\right)^{2}+\left(\bar{q}_{L} U P_{-} \mathcal{F}\left(\mathcal{M}_{d}\right) q_{R}\right)^{2}+\left(\bar{l}_{L} U P_{-} \mathcal{F}\left(\mathcal{M}_{e}\right) l_{R}\right)^{2} \\
& +2\left(\bar{q}_{L} U P_{+} \mathcal{F}\left(\mathcal{M}_{u}\right) q_{R}\right)\left(\bar{q}_{L} U P_{-} \mathcal{F}\left(\mathcal{M}_{d}\right) q_{R}\right)+2\left(\bar{q}_{L} U P_{+} \mathcal{F}\left(\mathcal{M}_{u}\right) q_{R}\right)\left(\bar{l}_{L} U P_{-} \mathcal{F}\left(\mathcal{M}_{e}\right) l_{R}\right) \\
& +2\left(\bar{q}_{L} U P_{-} \mathcal{F}\left(\mathcal{M}_{d}\right) q_{R}\right)\left(\bar{l}_{L} U P_{-} \mathcal{F}\left(\mathcal{M}_{e}\right) l_{R}\right) \\
= & \mathcal{F}\left(\mathcal{M}_{u}\right) \otimes \mathcal{F}\left(\mathcal{M}_{u}\right) \mathcal{O}_{F Y 1}+\mathcal{F}\left(\mathcal{M}_{d}\right) \otimes \mathcal{F}\left(\mathcal{M}_{d}\right) \mathcal{O}_{F Y 3}+\mathcal{F}\left(\mathcal{M}_{e}\right) \otimes \mathcal{F}\left(\mathcal{M}_{e}\right) \mathcal{O}_{F Y 10} \\
& +2 \mathcal{F}\left(\mathcal{M}_{u}\right) \otimes \mathcal{F}\left(\mathcal{M}_{d}\right) \mathcal{O}_{S T 5}+2 \mathcal{F}\left(\mathcal{M}_{u}\right) \otimes \mathcal{F}\left(\mathcal{M}_{e}\right) \mathcal{O}_{S T 9}+2 \mathcal{F}\left(\mathcal{M}_{d}\right) \otimes \mathcal{F}\left(\mathcal{M}_{e}\right) \mathcal{O}_{F Y 7},
\end{aligned}
$$

and

$$
\begin{aligned}
\left(\bar{\psi}_{L} U T^{a} \mathcal{F}(\mathcal{M}) \psi_{R}\right)\left(\bar{\psi}_{L} U T^{a} \mathcal{F}(\mathcal{M}) \psi_{R}\right)= & \left(\bar{q}_{L} U T^{a} P_{+} \mathcal{F}\left(\mathcal{M}_{u}\right) q_{R}\right)\left(\bar{q}_{L} U T^{a} P_{+} \mathcal{F}\left(\mathcal{M}_{u}\right) q_{R}\right) \\
& +\left(\bar{q}_{L} U T^{a} P_{-} \mathcal{F}\left(\mathcal{M}_{d}\right) q_{R}\right)\left(\bar{q}_{L} U T^{a} P_{-} \mathcal{F}\left(\mathcal{M}_{d}\right) q_{R}\right) \\
& +\left(\bar{l}_{L} U T^{a} P_{-} \mathcal{F}\left(\mathcal{M}_{e}\right) l_{R}\right)\left(\bar{l}_{L} U T^{a} P_{-} \mathcal{F}\left(\mathcal{M}_{e}\right) l_{R}\right) \\
& +2\left(\bar{q}_{L} U T^{a} P_{+} \mathcal{F}\left(\mathcal{M}_{u}\right) q_{R}\right)\left(\bar{q}_{L} U T^{a} P_{-} \mathcal{F}\left(\mathcal{M}_{d}\right) q_{R}\right) \\
& +2\left(\bar{q}_{L} U T^{a} P_{+} \mathcal{F}\left(\mathcal{M}_{u}\right) q_{R}\right)\left(\bar{l}_{L} U T^{a} P_{-} \mathcal{F}\left(\mathcal{M}_{e}\right) l_{R}\right) \\
& +2\left(\bar{q}_{L} U T^{a} P_{-} \mathcal{F}\left(\mathcal{M}_{d}\right) q_{R}\right)\left(\bar{l}_{L} U T^{a} P_{-} \mathcal{F}\left(\mathcal{M}_{e}\right) l_{R}\right) .
\end{aligned}
$$

Next, since

$$
T^{a} \otimes T^{a}=\frac{1}{2} P_{12} \otimes P_{21}+\frac{1}{2} P_{21} \otimes P_{12}+\frac{1}{4} P_{+} \otimes P_{+}+\frac{1}{4} P_{-} \otimes P_{-}-\frac{1}{4} P_{+} \otimes P_{-}-\frac{1}{4} P_{-} \otimes P_{+}
$$


one has

$$
\begin{aligned}
& T^{a} P_{ \pm} \otimes T^{a} P_{ \pm}=\frac{1}{4} P_{ \pm} \otimes P_{ \pm}, \quad T^{a} P_{+} \otimes T^{a} P_{-}=\frac{1}{2} P_{21} \otimes P_{12}-\frac{1}{4} P_{+} \otimes P_{-} \\
& T^{a} P_{-} \otimes T^{a} P_{+}=\frac{1}{2} P_{12} \otimes P_{21}-\frac{1}{4} P_{-} \otimes P_{+},
\end{aligned}
$$

so that

$$
\begin{aligned}
\left(\bar{\psi}_{L} U T^{a} \mathcal{F}(\mathcal{M}) \psi_{R}\right)\left(\bar{\psi}_{L} U T^{a} \mathcal{F}(\mathcal{M}) \psi_{R}\right)= & \frac{1}{4}\left(\bar{q}_{L} U P_{+} \mathcal{F}\left(\mathcal{M}_{u}\right) q_{R}\right)\left(\bar{q}_{L} U P_{+} \mathcal{F}\left(\mathcal{M}_{u}\right) q_{R}\right) \\
& +\frac{1}{4}\left(\bar{q}_{L} U P_{-} \mathcal{F}\left(\mathcal{M}_{d}\right) q_{R}\right)\left(\bar{q}_{L} U P_{-} \mathcal{F}\left(\mathcal{M}_{d}\right) q_{R}\right) \\
& +\frac{1}{4}\left(\bar{l}_{L} U P_{-} \mathcal{F}\left(\mathcal{M}_{e}\right) l_{R}\right)\left(\bar{l}_{L} U P_{-} \mathcal{F}\left(\mathcal{M}_{e}\right) l_{R}\right) \\
& +\left(\bar{q}_{L} U P_{21} \mathcal{F}\left(\mathcal{M}_{u}\right) q_{R}\right)\left(\bar{q}_{L} U P_{12} \mathcal{F}\left(\mathcal{M}_{d}\right) q_{R}\right) \\
& -\frac{1}{2}\left(\bar{q}_{L} U P_{+} \mathcal{F}\left(\mathcal{M}_{u}\right) q_{R}\right)\left(\bar{q}_{L} U P_{-} \mathcal{F}\left(\mathcal{M}_{d}\right) q_{R}\right) \\
& +\left(\bar{q}_{L} U P_{21} \mathcal{F}\left(\mathcal{M}_{u}\right) q_{R}\right)\left(\bar{l}_{L} U P_{12} \mathcal{F}\left(\mathcal{M}_{e}\right) l_{R}\right) \\
& -\frac{1}{2}\left(\bar{q}_{L} U P_{+} \mathcal{F}\left(\mathcal{M}_{u}\right) q_{R}\right)\left(\bar{l}_{L} U P_{-} \mathcal{F}\left(\mathcal{M}_{e}\right) l_{R}\right) \\
& +\frac{1}{2}\left(\bar{q}_{L} U P_{-} \mathcal{F}\left(\mathcal{M}_{d}\right) q_{R}\right)\left(\bar{l}_{L} U P_{-} \mathcal{F}\left(\mathcal{M}_{e}\right) l_{R}\right) \\
= & \frac{1}{4}\left(\mathcal{M}_{u}\right) \otimes \mathcal{F}\left(\mathcal{M}_{u}\right) \mathcal{O}_{F Y 1}+\frac{1}{4} \mathcal{F}\left(\mathcal{M}_{d}\right) \otimes \mathcal{F}\left(\mathcal{M}_{d}\right) \mathcal{O}_{F Y 3} \\
& +\frac{1}{4} \mathcal{F}\left(\mathcal{M}_{e}\right) \otimes \mathcal{F}\left(\mathcal{M}_{e}\right) \mathcal{O}_{F Y 10}+\mathcal{F}\left(\mathcal{M}_{u}\right) \otimes \mathcal{F}\left(\mathcal{M}_{d}\right) \mathcal{O}_{S T 6} \\
& -\frac{1}{2} \mathcal{F}\left(\mathcal{M}_{u}\right) \otimes \mathcal{F}\left(\mathcal{M}_{d}\right) \mathcal{O}_{S T 5}+\mathcal{F}\left(\mathcal{M}_{u}\right) \otimes \mathcal{F}\left(\mathcal{M}_{e}\right) \mathcal{O}_{S T 10} \\
& -\frac{1}{2} \mathcal{F}\left(\mathcal{M}_{u}\right) \otimes \mathcal{F}\left(\mathcal{M}_{e}\right) \mathcal{O}_{S T 9}+\frac{1}{2} \mathcal{F}\left(\mathcal{M}_{d}\right) \otimes \mathcal{F}_{e}\left(\mathcal{M}_{e}\right) \mathcal{O}_{F Y 7}
\end{aligned}
$$

In turn, the structure $\left(\bar{\psi}_{L} U \mathcal{F}(\mathcal{M}) \psi_{R}\right)\left(\bar{\psi}_{R} \mathcal{F}(\mathcal{M})^{\dagger} U^{\dagger} \psi_{L}\right)$ can be simplified using Eq. (5.23):

$$
\begin{aligned}
\left(\bar{\psi}_{L} U \mathcal{F}(\mathcal{M}) \psi_{R}\right)\left(\bar{\psi}_{R} \mathcal{F}(\mathcal{M})^{\dagger} U^{\dagger} \psi_{L}\right)= & {\left[\left(\bar{q}_{L} U P_{+} \mathcal{F}\left(\mathcal{M}_{u}\right) q_{R}\right)+\left(\bar{q}_{L} U P_{-} \mathcal{F}\left(\mathcal{M}_{d}\right) q_{R}\right)+\left(\bar{l}_{L} U P_{-} \mathcal{F}\left(\mathcal{M}_{e}\right) l_{R}\right)\right] } \\
& \times\left[\left(\bar{q}_{R} \mathcal{F}\left(\mathcal{M}_{u}\right)^{\dagger} P_{+} U^{\dagger} q_{L}\right)+\left(\bar{q}_{R} \mathcal{F}\left(\mathcal{M}_{d}\right)^{\dagger} P_{-} U^{\dagger} q_{L}\right)+\left(\bar{l}_{R} \mathcal{F}\left(\mathcal{M}_{e}\right)^{\dagger} P_{-} U^{\dagger} l_{L}\right)\right] \\
= & \left(\bar{q}_{L} U P_{+} \mathcal{F}\left(\mathcal{M}_{u}\right) q_{R}\right)\left(\bar{q}_{R} \mathcal{F}\left(\mathcal{M}_{u}\right)^{\dagger} P_{+} U^{\dagger} q_{L}\right) \\
& +\left(\bar{q}_{L} U P_{-} \mathcal{F}\left(\mathcal{M}_{d}\right) q_{R}\right)\left(\bar{q}_{R} \mathcal{F}\left(\mathcal{M}_{d}\right)^{\dagger} P_{-} U^{\dagger} q_{L}\right) \\
& +\left(\bar{l}_{L} U P_{-} \mathcal{F}\left(\mathcal{M}_{e}\right) l_{R}\right)\left(\bar{l}_{R} \mathcal{F}\left(\mathcal{M}_{e}\right)^{\dagger} P_{-} U^{\dagger} l_{L}\right) \\
& +\left(\bar{q}_{L} U P_{+} \mathcal{F}\left(\mathcal{M}_{u}\right) q_{R}\right)\left(\bar{q}_{R} \mathcal{F}\left(\mathcal{M}_{d}\right)^{\dagger} P_{-} U^{\dagger} q_{L}\right) \\
& +\left(\bar{q}_{L} U P_{-} \mathcal{F}\left(\mathcal{M}_{d}\right) q_{R}\right)\left(\bar{q}_{R} \mathcal{F}\left(\mathcal{M}_{u}\right)^{\dagger} P_{+} U^{\dagger} q_{L}\right) \\
& +\left(\bar{q}_{L} U P_{+} \mathcal{F}\left(\mathcal{M}_{u}\right) q_{R}\right)\left(\bar{l}_{R} \mathcal{F}\left(\mathcal{M}_{e}\right)^{\dagger} P_{-} U^{\dagger} l_{L}\right) \\
& +\left(\bar{q}_{R} \mathcal{F}\left(\mathcal{M}_{u}\right)^{\dagger} P_{+} U^{\dagger} q_{L}\right)\left(\bar{l}_{L} U P_{-} \mathcal{F}\left(\mathcal{M}_{e}\right) l_{R}\right) \\
& +\left(\bar{q}_{L} U P_{-} \mathcal{F}\left(\mathcal{M}_{d}\right) q_{R}\right)\left(\bar{l}_{R} \mathcal{F}\left(\mathcal{M}_{e}\right)^{\dagger} P_{-} U^{\dagger} l_{L}\right) \\
& +\left(\bar{q}_{R} \mathcal{F}\left(\mathcal{M}_{d}\right)^{\dagger} P_{-} U^{\dagger} q_{L}\right)\left(\bar{l}_{L} U P_{-} \mathcal{F}\left(\mathcal{M}_{e}\right) l_{R}\right) .
\end{aligned}
$$

The terms obtained this way can be decomposed on the operator basis through the use of the Fierz identities 
$\left(\bar{q}_{L} U P_{+} \mathcal{F}\left(\mathcal{M}_{u}\right) q_{R}\right)\left(\bar{q}_{R} \mathcal{F}\left(\mathcal{M}_{u}\right)^{\dagger} P_{+} U^{\dagger} q_{L}\right)=-\mathcal{F}\left(\mathcal{M}_{u}\right) \tilde{\otimes} \mathcal{F}\left(\mathcal{M}_{u}\right)^{\dagger}\left[\frac{1}{12} \mathcal{O}_{L R 1}+\frac{1}{2} \mathcal{O}_{L R 2}+\frac{1}{6} \mathcal{O}_{L R 10}+\mathcal{O}_{L R 11}\right]$,

$\left(\bar{q}_{L} U P_{-} \mathcal{F}\left(\mathcal{M}_{d}\right) q_{R}\right)\left(\bar{q}_{R} \mathcal{F}\left(\mathcal{M}_{d}\right)^{\dagger} P_{-} U^{\dagger} q_{L}\right)=-\mathcal{F}\left(\mathcal{M}_{d}\right) \tilde{\otimes} \mathcal{F}\left(\mathcal{M}_{d}\right)^{\dagger}\left[\frac{1}{12} \mathcal{O}_{L R 3}+\frac{1}{2} \mathcal{O}_{L R 4}-\frac{1}{6} \mathcal{O}_{L R 12}-\mathcal{O}_{L R 13}\right]$,

$\left(\bar{l}_{L} U P_{-} \mathcal{F}\left(\mathcal{M}_{e}\right) l_{R}\right)\left(\bar{l}_{R} \mathcal{F}\left(\mathcal{M}_{e}\right)^{\dagger} P_{-} U^{\dagger} l_{L}\right)=-\mathcal{F}\left(\mathcal{M}_{e}\right) \tilde{\otimes} \mathcal{F}\left(\mathcal{M}_{e}\right)^{\dagger}\left[\frac{1}{4} \mathcal{O}_{L R 8}-\frac{1}{2} \mathcal{O}_{L R 17}\right]$,

$\left(\bar{q}_{L} U P_{-} \mathcal{F}\left(\mathcal{M}_{d}\right) q_{R}\right)\left(\bar{l}_{R} \mathcal{F}\left(\mathcal{M}_{e}\right)^{\dagger} P_{-} U^{\dagger} l_{L}\right)=-\mathcal{F}\left(\mathcal{M}_{d}\right) \tilde{\otimes} \mathcal{F}\left(\mathcal{M}_{e}\right)^{\dagger}\left[\frac{1}{4} \mathcal{O}_{L R 9}-\frac{1}{2} \mathcal{O}_{L R 18}\right]$,

so that

$$
\begin{aligned}
\left(\bar{\psi}_{L} U \mathcal{F}(\mathcal{M}) \psi_{R}\right)\left(\bar{\psi}_{R} \mathcal{F}(\mathcal{M})^{\dagger} U^{\dagger} \psi_{L}\right)= & -\mathcal{F}\left(\mathcal{M}_{u}\right) \tilde{\otimes} \mathcal{F}\left(\mathcal{M}_{u}\right)^{\dagger}\left[\frac{1}{12} \mathcal{O}_{L R 1}+\frac{1}{2} \mathcal{O}_{L R 2}+\frac{1}{6} \mathcal{O}_{L R 10}+\mathcal{O}_{L R 11}\right] \\
& -\mathcal{F}\left(\mathcal{M}_{d}\right) \tilde{\otimes} \mathcal{F}\left(\mathcal{M}_{d}\right)^{\dagger}\left[\frac{1}{12} \mathcal{O}_{L R 3}+\frac{1}{2} \mathcal{O}_{L R 4}-\frac{1}{6} \mathcal{O}_{L R 12}-\mathcal{O}_{L R 13}\right] \\
& -\mathcal{F}\left(\mathcal{M}_{e}\right) \tilde{\otimes} \mathcal{F}\left(\mathcal{M}_{e}\right)^{\dagger}\left[\frac{1}{4} \mathcal{O}_{L R 8}-\frac{1}{2} \mathcal{O}_{L R 17}\right] \\
& +\mathcal{F}\left(\mathcal{M}_{d}\right) \otimes \mathcal{F}\left(\mathcal{M}_{u}\right)^{\dagger} \mathcal{O}_{F Y 5}+\mathcal{F}\left(\mathcal{M}_{u}\right) \otimes \mathcal{F}\left(\mathcal{M}_{d}\right)^{\dagger} \mathcal{O}_{F Y 5}^{\dagger} \\
& +\mathcal{F}\left(\mathcal{M}_{e}\right) \otimes \mathcal{F}\left(\mathcal{M}_{u}\right)^{\dagger} \mathcal{O}_{F Y 9}+\mathcal{F}\left(\mathcal{M}_{u}\right) \otimes \mathcal{F}\left(\mathcal{M}_{e}\right)^{\dagger} \mathcal{O}_{F Y 9}^{\dagger} \\
& -\mathcal{F}\left(\mathcal{M}_{d}\right) \tilde{\otimes} \mathcal{F}\left(\mathcal{M}_{e}\right)^{\dagger}\left[\frac{1}{4} \mathcal{O}_{L R 9}-\frac{1}{2} \mathcal{O}_{L R 18}\right] \\
& -\mathcal{F}\left(\mathcal{M}_{e}\right) \tilde{\otimes} \mathcal{F}\left(\mathcal{M}_{d}\right)^{\dagger}\left[\frac{1}{4} \mathcal{O}_{L R 9}^{\dagger}-\frac{1}{2} \mathcal{O}_{L R 18}^{\dagger}\right] .
\end{aligned}
$$

Finally, one has

$$
\begin{aligned}
\left(\bar{\psi}_{L} U T^{a} \mathcal{F}(\mathcal{M}) \psi_{R}\right)\left(\bar{\psi}_{R} \mathcal{F}(\mathcal{M})^{\dagger} T^{a} U^{\dagger} \psi_{L}\right)= & {\left[\left(\bar{q}_{L} U T^{a} P_{+} \mathcal{F}\left(\mathcal{M}_{u}\right) q_{R}\right)+\left(\bar{q}_{L} U T^{a} P_{-} \mathcal{F}\left(\mathcal{M}_{d}\right) q_{R}\right)\right.} \\
& \left.+\left(\bar{l}_{L} U T^{a} P_{-} \mathcal{F}\left(\mathcal{M}_{e}\right) l_{R}\right)\right] \times\left[\left(\bar{q}_{R} \mathcal{F}\left(\mathcal{M}_{u}\right)^{\dagger} P_{+} T^{a} U^{\dagger} q_{L}\right)\right. \\
& \left.+\left(\bar{q}_{R} \mathcal{F}\left(\mathcal{M}_{d}\right)^{\dagger} P_{-} T^{a} U^{\dagger} q_{L}\right)+\left(\bar{l}_{R} \mathcal{F}\left(\mathcal{M}_{e}\right)^{\dagger} P_{-} T^{a} U^{\dagger} l_{L}\right)\right] \\
= & \left(\bar{q}_{L} U T^{a} P_{+} \mathcal{F}\left(\mathcal{M}_{u}\right) q_{R}\right)\left(\bar{q}_{R} \mathcal{F}\left(\mathcal{M}_{u}\right)^{\dagger} P_{+} T^{a} U^{\dagger} q_{L}\right) \\
& +\left(\bar{q}_{L} U T^{a} P_{-} \mathcal{F}\left(\mathcal{M}_{d}\right) q_{R}\right)\left(\bar{q}_{R} \mathcal{F}\left(\mathcal{M}_{d}\right)^{\dagger} P_{-} T^{a} U^{\dagger} q_{L}\right) \\
& +\left(\bar{l}_{L} U T^{a} P_{-} \mathcal{F}\left(\mathcal{M}_{e}\right) l_{R}\right)\left(\bar{l}_{R} \mathcal{F}\left(\mathcal{M}_{e}\right)^{\dagger} P_{-} T^{a} U^{\dagger} l_{L}\right) \\
& +\left(\bar{q}_{L} U T^{a} P_{+} \mathcal{F}\left(\mathcal{M}_{u}\right) q_{R}\right)\left(\bar{q}_{R} \mathcal{F}\left(\mathcal{M}_{d}\right)^{\dagger} P_{-} T^{a} U^{\dagger} q_{L}\right) \\
& +\left(\bar{q}_{L} U T^{a} P_{-} \mathcal{F}\left(\mathcal{M}_{d}\right) q_{R}\right)\left(\bar{q}_{R} \mathcal{F}\left(\mathcal{M}_{u}\right)^{\dagger} P_{+} T^{a} U^{\dagger} q_{L}\right) \\
& +\left(\bar{q}_{L} U T^{a} P_{+} \mathcal{F}\left(\mathcal{M}_{u}\right) q_{R}\right)\left(\bar{l}_{R} \mathcal{F}\left(\mathcal{M}_{e}\right)^{\dagger} P_{-} T^{a} U^{\dagger} l_{L}\right) \\
& +\left(\bar{l}_{L} U T^{a} P_{-} \mathcal{F}\left(\mathcal{M}_{e}\right) l_{R}\right)\left(\bar{q}_{R} \mathcal{F}\left(\mathcal{M}_{u}\right)^{\dagger} P_{+} T^{a} U^{\dagger} q_{L}\right) \\
& +\left(\bar{q}_{L} U T^{a} P_{-} \mathcal{F}\left(\mathcal{M}_{d}\right) q_{R}\right)\left(\bar{l}_{R} \mathcal{F}\left(\mathcal{M}_{e}\right)^{\dagger} P_{-} T^{a} U^{\dagger} l_{L}\right) \\
& +\left(\bar{l}_{L} U T^{a} P_{-} \mathcal{F}\left(\mathcal{M}_{e}\right) l_{R}\right)\left(\bar{q}_{R} \mathcal{F}\left(\mathcal{M}_{d}\right)^{\dagger} P_{-} T^{a} U^{\dagger} q_{L}\right) .
\end{aligned}
$$

Using now

$$
\begin{aligned}
& T^{a} P_{+} \otimes P_{+} T^{a}=\frac{1}{2} P_{21} \otimes P_{12}+\frac{1}{4} P_{+} \otimes P_{+}, \quad T^{a} P_{-} \otimes P_{-} T^{a}=\frac{1}{2} P_{12} \otimes P_{21}+\frac{1}{4} P_{-} \otimes P_{-}, \\
& T^{a} P_{ \pm} \otimes P_{\mp} T^{a}=-\frac{1}{4} P_{ \pm} \otimes P_{\mp},
\end{aligned}
$$


together with the previous Fierz identities and the following ones:

$\left(\bar{q}_{L} U P_{12} \mathcal{F}\left(\mathcal{M}_{d}\right) q_{R}\right)\left(\bar{q}_{R} \mathcal{F}\left(\mathcal{M}_{d}\right)^{\dagger} P_{21} U^{\dagger} q_{L}\right)=-\mathcal{F}\left(\mathcal{M}_{d}\right) \tilde{\otimes} \mathcal{F}\left(\mathcal{M}_{d}\right)^{\dagger}\left[\frac{1}{12} \mathcal{O}_{L R 3}+\frac{1}{2} \mathcal{O}_{L R 4}+\frac{1}{6} \mathcal{O}_{L R 12}+\mathcal{O}_{L R 13}\right]$,

$\left(\bar{q}_{L} U P_{21} \mathcal{F}\left(\mathcal{M}_{u}\right) q_{R}\right)\left(\bar{q}_{R} \mathcal{F}\left(\mathcal{M}_{u}\right)^{\dagger} P_{12} U^{\dagger} q_{L}\right)=-\mathcal{F}\left(\mathcal{M}_{u}\right) \tilde{\otimes} \mathcal{F}\left(\mathcal{M}_{u}\right)^{\dagger}\left[\frac{1}{12} \mathcal{O}_{L R 1}+\frac{1}{2} \mathcal{O}_{L R 2}-\frac{1}{6} \mathcal{O}_{L R 10}-\mathcal{O}_{L R 11}\right]$,

$\left(\bar{l}_{L} U P_{12} \mathcal{F}\left(\mathcal{M}_{e}\right) l_{R}\right)\left(\bar{l}_{R} \mathcal{F}\left(\mathcal{M}_{e}\right)^{\dagger} P_{21} U^{\dagger} l_{L}\right)=-\mathcal{F}\left(\mathcal{M}_{e}\right) \tilde{\otimes} \mathcal{F}\left(\mathcal{M}_{e}\right)^{\dagger}\left[\frac{1}{4} \mathcal{O}_{L R 8}+\frac{1}{2} \mathcal{O}_{L R 17}\right]$,

$\left(\bar{q}_{L} U P_{12} \mathcal{F}\left(\mathcal{M}_{d}\right) q_{R}\right)\left(\bar{l}_{R} \mathcal{F}\left(\mathcal{M}_{e}\right)^{\dagger} P_{21} U^{\dagger} l_{L}\right)=-\mathcal{F}\left(\mathcal{M}_{d}\right) \tilde{\otimes} \mathcal{F}\left(\mathcal{M}_{e}\right)^{\dagger}\left[\frac{1}{4} \mathcal{O}_{L R 9}+\frac{1}{2} \mathcal{O}_{L R 18}\right]$,

one obtains

$$
\begin{aligned}
& \left(\bar{\psi}_{L} U T^{a} \mathcal{F}(\mathcal{M}) \psi_{R}\right)\left(\bar{\psi}_{R} \mathcal{F}(\mathcal{M})^{\dagger} T^{a} U^{\dagger} \psi_{L}\right) \\
& =\frac{1}{2}\left(\bar{q}_{L} U P_{21} \mathcal{F}\left(\mathcal{M}_{u}\right) q_{R}\right)\left(\bar{q}_{R} \mathcal{F}\left(\mathcal{M}_{u}\right)^{\dagger} P_{12} U^{\dagger} q_{L}\right)+\frac{1}{4}\left(\bar{q}_{L} U P_{+} \mathcal{F}\left(\mathcal{M}_{u}\right) q_{R}\right)\left(\bar{q}_{R} \mathcal{F}\left(\mathcal{M}_{u}\right)^{\dagger} P_{+} U^{\dagger} q_{L}\right) \\
& +\frac{1}{2}\left(\bar{q}_{L} U P_{12} \mathcal{F}\left(\mathcal{M}_{d}\right) q_{R}\right)\left(\bar{q}_{R} \mathcal{F}\left(\mathcal{M}_{d}\right)^{\dagger} P_{21} U^{\dagger} q_{L}\right)+\frac{1}{4}\left(\bar{q}_{L} U P_{-} \mathcal{F}\left(\mathcal{M}_{d}\right) q_{R}\right)\left(\bar{q}_{R} \mathcal{F}\left(\mathcal{M}_{d}\right)^{\dagger} P_{-} U^{\dagger} q_{L}\right) \\
& +\frac{1}{2}\left(\bar{l}_{L} U P_{12} \mathcal{F}\left(\mathcal{M}_{e}\right) l_{R}\right)\left(\bar{l}_{R} \mathcal{F}\left(\mathcal{M}_{e}\right)^{\dagger} P_{21} U^{\dagger} l_{L}\right)+\frac{1}{4}\left(\bar{l}_{L} U P_{-} \mathcal{F}\left(\mathcal{M}_{e}\right) l_{R}\right)\left(\bar{l}_{R} \mathcal{F}\left(\mathcal{M}_{e}\right)^{\dagger} P_{-} U^{\dagger} l_{L}\right) \\
& -\frac{1}{4}\left(\bar{q}_{L} U P_{+} \mathcal{F}\left(\mathcal{M}_{u}\right) q_{R}\right)\left(\bar{q}_{R} \mathcal{F}\left(\mathcal{M}_{d}\right)^{\dagger} P_{-} U^{\dagger} q_{L}\right)-\frac{1}{4}\left(\bar{q}_{L} U P_{-} \mathcal{F}\left(\mathcal{M}_{d}\right) q_{R}\right)\left(\bar{q}_{R} \mathcal{F}\left(\mathcal{M}_{u}\right)^{\dagger} P_{+} U^{\dagger} q_{L}\right) \\
& -\frac{1}{4}\left(\bar{q}_{L} U P_{+} \mathcal{F}\left(\mathcal{M}_{u}\right) q_{R}\right)\left(\bar{l}_{R} \mathcal{F}\left(\mathcal{M}_{e}\right)^{\dagger} P_{-} U^{\dagger} l_{L}\right)-\frac{1}{4}\left(\bar{l}_{L} U P_{-} \mathcal{F}\left(\mathcal{M}_{e}\right) l_{R}\right)\left(\bar{q}_{R} \mathcal{F}\left(\mathcal{M}_{u}\right)^{\dagger} P_{+} U^{\dagger} q_{L}\right) \\
& +\frac{1}{2}\left(\bar{q}_{L} U P_{12} \mathcal{F}\left(\mathcal{M}_{d}\right) q_{R}\right)\left(\bar{l}_{R} \mathcal{F}\left(\mathcal{M}_{e}\right)^{\dagger} P_{21} U^{\dagger} l_{L}\right)+\frac{1}{4}\left(\bar{q}_{L} U P_{-} \mathcal{F}\left(\mathcal{M}_{d}\right) q_{R}\right)\left(\bar{l}_{R} \mathcal{F}\left(\mathcal{M}_{e}\right)^{\dagger} P_{-} U^{\dagger} l_{L}\right) \\
& +\frac{1}{2}\left(\bar{l}_{L} U P_{12} \mathcal{F}\left(\mathcal{M}_{e}\right) l_{R}\right)\left(\bar{q}_{R} \mathcal{F}\left(\mathcal{M}_{d}\right)^{\dagger} P_{21} U^{\dagger} q_{L}\right)+\frac{1}{4}\left(\bar{l}_{L} U P_{-} \mathcal{F}\left(\mathcal{M}_{e}\right) l_{R}\right)\left(\bar{q}_{R} \mathcal{F}\left(\mathcal{M}_{d}\right)^{\dagger} P_{-} U^{\dagger} q_{L}\right) \\
& =-\mathcal{F}\left(\mathcal{M}_{u}\right) \tilde{\otimes} \mathcal{F}\left(\mathcal{M}_{u}\right)^{\dagger}\left[\frac{1}{24} \mathcal{O}_{L R 1}+\frac{1}{4} \mathcal{O}_{L R 2}-\frac{1}{12} \mathcal{O}_{L R 10}-\frac{1}{2} \mathcal{O}_{L R 11}\right] \\
& -\mathcal{F}\left(\mathcal{M}_{u}\right) \tilde{\otimes} \mathcal{F}\left(\mathcal{M}_{u}\right)^{\dagger}\left[\frac{1}{48} \mathcal{O}_{L R 1}+\frac{1}{8} \mathcal{O}_{L R 2}+\frac{1}{24} \mathcal{O}_{L R 10}+\frac{1}{4} \mathcal{O}_{L R 11}\right] \\
& -\mathcal{F}\left(\mathcal{M}_{d}\right) \tilde{\otimes} \mathcal{F}\left(\mathcal{M}_{d}\right)^{\dagger}\left[\frac{1}{24} \mathcal{O}_{L R 3}+\frac{1}{4} \mathcal{O}_{L R 4}+\frac{1}{12} \mathcal{O}_{L R 12}+\frac{1}{2} \mathcal{O}_{L R 13}\right] \\
& -\mathcal{F}\left(\mathcal{M}_{d}\right) \tilde{\otimes} \mathcal{F}\left(\mathcal{M}_{d}\right)^{\dagger}\left[\frac{1}{48} \mathcal{O}_{L R 3}+\frac{1}{8} \mathcal{O}_{L R 4}-\frac{1}{24} \mathcal{O}_{L R 12}-\frac{1}{4} \mathcal{O}_{L R 13}\right] \\
& -\mathcal{F}\left(\mathcal{M}_{e}\right) \tilde{\otimes} \mathcal{F}\left(\mathcal{M}_{e}\right)^{\dagger}\left[\frac{1}{8} \mathcal{O}_{L R 8}+\frac{1}{4} \mathcal{O}_{L R 17}\right]-\mathcal{F}\left(\mathcal{M}_{e}\right) \tilde{\otimes} \mathcal{F}\left(\mathcal{M}_{e}\right)^{\dagger}\left[\frac{1}{16} \mathcal{O}_{L R 8}-\frac{1}{8} \mathcal{O}_{L R 17}\right] \\
& -\mathcal{F}\left(\mathcal{M}_{d}\right) \tilde{\otimes} \mathcal{F}\left(\mathcal{M}_{e}\right)^{\dagger}\left[\frac{1}{8} \mathcal{O}_{L R 9}+\frac{1}{4} \mathcal{O}_{L R 18}\right]-\frac{1}{4} \mathcal{F}\left(\mathcal{M}_{d}\right) \otimes \mathcal{F}\left(\mathcal{M}_{u}\right)^{\dagger} \mathcal{O}_{F Y 5} \\
& -\mathcal{F}\left(\mathcal{M}_{d}\right) \tilde{\otimes} \mathcal{F}\left(\mathcal{M}_{e}\right)^{\dagger}\left[\frac{1}{16} \mathcal{O}_{L R 9}-\frac{1}{8} \mathcal{O}_{L R 18}\right]-\frac{1}{4} \mathcal{F}\left(\mathcal{M}_{u}\right) \otimes \mathcal{F}\left(\mathcal{M}_{d}\right)^{\dagger} \mathcal{O}_{F Y 5}^{\dagger} \\
& -\mathcal{F}\left(\mathcal{M}_{e}\right) \tilde{\otimes} \mathcal{F}\left(\mathcal{M}_{d}\right)^{\dagger}\left[\frac{1}{8} \mathcal{O}_{L R 9}^{\dagger}+\frac{1}{4} \mathcal{O}_{L R 18}^{\dagger}\right]-\frac{1}{4} \mathcal{F}\left(\mathcal{M}_{e}\right) \otimes \mathcal{F}\left(\mathcal{M}_{u}\right)^{\dagger} \mathcal{O}_{F Y 9} \\
& -\mathcal{F}\left(\mathcal{M}_{e}\right) \tilde{\otimes} \mathcal{F}\left(\mathcal{M}_{d}\right)^{\dagger}\left[\frac{1}{16} \mathcal{O}_{L R 9}^{\dagger}-\frac{1}{8} \mathcal{O}_{L R 18}^{\dagger}\right]-\frac{1}{4} \mathcal{F}\left(\mathcal{M}_{u}\right) \otimes \mathcal{F}\left(\mathcal{M}_{e}\right)^{\dagger} \mathcal{O}_{F Y 9}^{\dagger} .
\end{aligned}
$$


These divergences are removed through the renormalization of the operators $\mathcal{O}_{F Y i}, \mathcal{O}_{S T i}$, and $\mathcal{O}_{L R i}$ of $\mathcal{L}_{\psi^{4} U h}$. Explicitly, one has (recall that the functions $F_{F Y i}, F_{S T i}$, and $F_{L R i}$ are actually rank-four tensors in generation space)

$$
\begin{aligned}
& \stackrel{\circ}{F}_{F Y i}(\eta)=F_{F Y i}(\eta ; \mu)+\frac{\Gamma_{F Y i}(\eta)}{16 \pi^{2}} \mu^{d-4}\left[\frac{1}{d-4}-\frac{1}{2}\left(\ln 4 \pi-\gamma_{E}\right)\right], \\
& \stackrel{\circ}{F}_{S T i}(\eta)=F_{S T i}(\eta ; \mu)+\frac{\Gamma_{S T i}(\eta)}{16 \pi^{2}} \mu^{d-4}\left[\frac{1}{d-4}-\frac{1}{2}\left(\ln 4 \pi-\gamma_{E}\right)\right], \\
& \stackrel{\circ}{F}_{L R i}(\eta)=F_{L R i}(\eta ; \mu)+\frac{\Gamma_{L R i}(\eta)}{16 \pi^{2}} \mu^{d-4}\left[\frac{1}{d-4}-\frac{1}{2}\left(\ln 4 \pi-\gamma_{E}\right)\right],
\end{aligned}
$$

with

$$
\begin{aligned}
& \Gamma_{F Y 1}=\frac{3 F^{-2}}{2 v^{4}}\left(\frac{F^{\prime}}{2} \mathcal{M}_{u}^{\prime}-\mathcal{M}_{u}\right) \otimes\left(\frac{F^{\prime}}{2} \mathcal{M}_{u}^{\prime}-\mathcal{M}_{u}\right)+\frac{1}{2 v^{4}} \mathcal{M}_{u}^{\prime \prime} \otimes \mathcal{M}_{u}^{\prime \prime}-\frac{1}{v^{4}}\left(F^{-1 / 2} \mathcal{M}_{u}\right)^{\prime} \otimes\left(F^{-1 / 2} \mathcal{M}_{u}\right)^{\prime} \\
& \Gamma_{F Y 3}=\frac{3 F^{-2}}{2 v^{4}}\left(\frac{F^{\prime}}{2} \mathcal{M}_{d}^{\prime}-\mathcal{M}_{d}\right) \otimes\left(\frac{F^{\prime}}{2} \mathcal{M}_{d}^{\prime}-\mathcal{M}_{d}\right)+\frac{1}{2 v^{4}} \mathcal{M}_{d}^{\prime \prime} \otimes \mathcal{M}_{d}^{\prime \prime}-\frac{1}{v^{4}}\left(F^{-1 / 2} \mathcal{M}_{d}\right)^{\prime} \otimes\left(F^{-1 / 2} \mathcal{M}_{d}\right)^{\prime}, \\
& \Gamma_{F Y 5}=\frac{3 F^{-2}}{v^{4}}\left(\frac{F^{\prime}}{2} \mathcal{M}_{d}^{\prime}-\mathcal{M}_{d}\right) \otimes\left(\frac{F^{\prime}}{2} \mathcal{M}_{u}^{\dagger \prime}-\mathcal{M}_{u}^{\dagger}\right)+\frac{1}{v^{4}} \mathcal{M}_{d}^{\prime \prime} \otimes \mathcal{M}_{u}^{\dagger \prime \prime}-\frac{2}{v^{4}}\left(F^{-1 / 2} \mathcal{M}_{d}\right)^{\prime} \otimes\left(F^{-1 / 2} \mathcal{M}_{u}^{\dagger}\right)^{\prime}, \\
& \Gamma_{F Y 7}=\frac{3 F^{-2}}{v^{4}}\left(\frac{F^{\prime}}{2} \mathcal{M}_{d}^{\prime}-\mathcal{M}_{d}\right) \otimes\left(\frac{F^{\prime}}{2} \mathcal{M}_{e}^{\prime}-\mathcal{M}_{e}\right)+\frac{1}{v^{4}} \mathcal{M}_{d}^{\prime \prime} \otimes \mathcal{M}_{e}^{\prime \prime}-\frac{2}{v^{4}}\left(F^{-1 / 2} \mathcal{M}_{d}\right)^{\prime} \otimes\left(F^{-1 / 2} \mathcal{M}_{e}\right)^{\prime}, \\
& \Gamma_{F Y 9}=\frac{3 F^{-2}}{v^{4}}\left(\frac{F^{\prime}}{2} \mathcal{M}_{e}^{\prime}-\mathcal{M}_{e}\right) \otimes\left(\frac{F^{\prime}}{2} \mathcal{M}_{u}^{\dagger \prime}-\mathcal{M}_{u}^{\dagger}\right)+\frac{1}{v^{4}} \mathcal{M}_{e}^{\prime \prime} \otimes \mathcal{M}_{u}^{\dagger \prime \prime}-\frac{2}{v^{4}}\left(F^{-1 / 2} \mathcal{M}_{e}\right)^{\prime} \otimes\left(F^{-1 / 2} \mathcal{M}_{u}^{\dagger}\right)^{\prime}, \\
& \Gamma_{F Y 10}=\frac{3 F^{-2}}{2 v^{4}}\left(\frac{F^{\prime}}{2} \mathcal{M}_{e}^{\prime}-\mathcal{M}_{e}\right) \otimes\left(\frac{F^{\prime}}{2} \mathcal{M}_{e}^{\prime}-\mathcal{M}_{e}\right)+\frac{1}{2 v^{4}} \mathcal{M}_{e}^{\prime \prime} \otimes \mathcal{M}_{e}^{\prime \prime}-\frac{1}{v^{4}}\left(F^{-1 / 2} \mathcal{M}_{e}\right)^{\prime} \otimes\left(F^{-1 / 2} \mathcal{M}_{e}\right)^{\prime}, \\
& \Gamma_{S T 5}=\frac{3 F^{-2}}{v^{4}}\left(\frac{F^{\prime}}{2} \mathcal{M}_{u}^{\prime}-\mathcal{M}_{u}\right) \otimes\left(\frac{F^{\prime}}{2} \mathcal{M}_{d}^{\prime}-\mathcal{M}_{d}\right)+\frac{1}{v^{4}} \mathcal{M}_{u}^{\prime \prime} \otimes \mathcal{M}_{d}^{\prime \prime}+\frac{2}{v^{4}}\left(F^{-1 / 2} \mathcal{M}_{u}\right)^{\prime} \otimes\left(F^{-1 / 2} \mathcal{M}_{d}\right)^{\prime}, \\
& \Gamma_{S T 6}=-\frac{4}{v^{4}}\left(F^{-1 / 2} \mathcal{M}_{u}\right)^{\prime} \otimes\left(F^{-1 / 2} \mathcal{M}_{d}\right)^{\prime}, \\
& \Gamma_{S T 9}=\frac{3 F^{-2}}{v^{4}}\left(\frac{F^{\prime}}{2} \mathcal{M}_{u}^{\prime}-\mathcal{M}_{u}\right) \otimes\left(\frac{F^{\prime}}{2} \mathcal{M}_{e}^{\prime}-\mathcal{M}_{e}\right)+\frac{1}{v^{4}} \mathcal{M}_{u}^{\prime \prime} \otimes \mathcal{M}_{e}^{\prime \prime}+\frac{2}{v^{4}}\left(F^{-1 / 2} \mathcal{M}_{u}\right)^{\prime} \otimes\left(F^{-1 / 2} \mathcal{M}_{e}\right)^{\prime}, \\
& \Gamma_{S T 10}=-\frac{4}{v^{4}}\left(F^{-1 / 2} \mathcal{M}_{u}\right)^{\prime} \otimes\left(F^{-1 / 2} \mathcal{M}_{e}\right)^{\prime}, \\
& \Gamma_{L R 1}=-\frac{F^{-2}}{4 v^{4}}\left(\frac{F^{\prime}}{2} \mathcal{M}_{u}^{\prime}-\mathcal{M}_{u}\right) \tilde{\otimes}\left(\frac{F^{\prime}}{2} \mathcal{M}_{u}^{\dagger \prime}-\mathcal{M}_{u}^{\dagger}\right)-\frac{1}{12 v^{4}} \mathcal{M}_{u}^{\prime \prime} \tilde{\otimes} \mathcal{M}_{u}^{\dagger \prime \prime}-\frac{1}{2 v^{4}}\left(F^{-1 / 2} \mathcal{M}_{u}\right)^{\prime} \tilde{\otimes}\left(F^{-1 / 2} \mathcal{M}_{u}^{\dagger}\right)^{\prime}, \\
& \Gamma_{L R 2}=-\frac{3 F^{-2}}{2 v^{4}}\left(\frac{F^{\prime}}{2} \mathcal{M}_{u}^{\prime}-\mathcal{M}_{u}\right) \tilde{\otimes}\left(\frac{F^{\prime}}{2} \mathcal{M}_{u}^{\dagger \prime}-\mathcal{M}_{u}^{\dagger}\right)-\frac{1}{2 v^{4}} \mathcal{M}_{u}^{\prime \prime} \tilde{\otimes} \mathcal{M}_{u}^{\dagger \prime \prime}-\frac{3}{v^{4}}\left(F^{-1 / 2} \mathcal{M}_{u}\right)^{\prime} \tilde{\otimes}\left(F^{-1 / 2} \mathcal{M}_{u}^{\dagger}\right)^{\prime}, \\
& \Gamma_{L R 3}=-\frac{F^{-2}}{4 v^{4}}\left(\frac{F^{\prime}}{2} \mathcal{M}_{d}^{\prime}-\mathcal{M}_{d}\right) \tilde{\otimes}\left(\frac{F^{\prime}}{2} \mathcal{M}_{d}^{\dagger \prime}-\mathcal{M}_{d}^{\dagger}\right)-\frac{1}{12 v^{4}} \mathcal{M}_{d}^{\prime \prime} \tilde{\otimes} \mathcal{M}_{d}^{\dagger \prime \prime}-\frac{1}{2 v^{4}}\left(F^{-1 / 2} \mathcal{M}_{d}\right)^{\prime} \tilde{\otimes}\left(F^{-1 / 2} \mathcal{M}_{d}^{\dagger}\right)^{\prime}, \\
& \Gamma_{L R 4}=-\frac{3 F^{-2}}{2 v^{4}}\left(\frac{F^{\prime}}{2} \mathcal{M}_{d}^{\prime}-\mathcal{M}_{d}\right) \tilde{\otimes}\left(\frac{F^{\prime}}{2} \mathcal{M}_{d}^{\dagger \prime}-\mathcal{M}_{d}^{\dagger}\right)-\frac{1}{2 v^{4}} \mathcal{M}_{d}^{\prime \prime} \tilde{\otimes} \mathcal{M}_{d}^{\dagger \prime \prime}-\frac{3}{v^{4}}\left(F^{-1 / 2} \mathcal{M}_{d}\right)^{\prime} \tilde{\otimes}\left(F^{-1 / 2} \mathcal{M}_{d}^{\dagger}\right)^{\prime}, \\
& \Gamma_{L R 8}=-\frac{3 F^{-2}}{4 v^{4}}\left(\frac{F^{\prime}}{2} \mathcal{M}_{e}^{\prime}-\mathcal{M}_{e}\right) \tilde{\otimes}\left(\frac{F^{\prime}}{2} \mathcal{M}_{e}^{\dagger \prime}-\mathcal{M}_{e}^{\dagger}\right)-\frac{1}{4 v^{4}} \mathcal{M}_{e}^{\prime \prime} \tilde{\otimes} \mathcal{M}_{e}^{\dagger \prime \prime}-\frac{3}{2 v^{4}}\left(F^{-1 / 2} \mathcal{M}_{e}\right)^{\prime} \tilde{\otimes}\left(F^{-1 / 2} \mathcal{M}_{e}^{\dagger}\right)^{\prime} \text {, }
\end{aligned}
$$




$$
\begin{aligned}
\Gamma_{L R 9}= & -\frac{3 F^{-2}}{4 v^{4}}\left(\frac{F^{\prime}}{2} \mathcal{M}_{d}^{\prime}-\mathcal{M}_{d}\right) \tilde{\otimes}\left(\frac{F^{\prime}}{2} \mathcal{M}_{e}^{\dagger \prime}-\mathcal{M}_{e}^{\dagger}\right)-\frac{1}{4 v^{4}} \mathcal{M}_{d}^{\prime \prime} \tilde{\otimes} \mathcal{M}_{e}^{\dagger \prime \prime}-\frac{3}{2 v^{4}}\left(F^{-1 / 2} \mathcal{M}_{d}\right)^{\prime} \tilde{\otimes}\left(F^{-1 / 2} \mathcal{M}_{e}^{\dagger}\right)^{\prime}, \\
\Gamma_{L R 10}= & -\frac{F^{-2}}{2 v^{4}}\left(\frac{F^{\prime}}{2} \mathcal{M}_{u}^{\prime}-\mathcal{M}_{u}\right) \tilde{\otimes}\left(\frac{F^{\prime}}{2} \mathcal{M}_{u}^{\dagger \prime}-\mathcal{M}_{u}^{\dagger}\right)-\frac{1}{6 v^{4}} \mathcal{M}_{u}^{\prime \prime} \tilde{\otimes} \mathcal{M}_{u}^{\dagger \prime \prime}+\frac{1}{3 v^{4}}\left(F^{-1 / 2} \mathcal{M}_{u}\right)^{\prime} \tilde{\otimes}\left(F^{-1 / 2} \mathcal{M}_{u}^{\dagger}\right)^{\prime}, \\
\Gamma_{L R 11}= & -\frac{3 F^{-2}}{v^{4}}\left(\frac{F^{\prime}}{2} \mathcal{M}_{u}^{\prime}-\mathcal{M}_{u}\right) \tilde{\otimes}\left(\frac{F^{\prime}}{2} \mathcal{M}_{u}^{\dagger \prime}-\mathcal{M}_{u}^{\dagger}\right)-\frac{1}{v^{4}} \mathcal{M}_{u}^{\prime \prime} \tilde{\otimes} \mathcal{M}_{u}^{\dagger \prime \prime}+\frac{2}{v^{4}}\left(F^{-1 / 2} \mathcal{M}_{u}\right)^{\prime} \tilde{\otimes}\left(F^{-1 / 2} \mathcal{M}_{u}^{\dagger}\right)^{\prime}, \\
\Gamma_{L R 12}= & \frac{F^{-2}}{2 v^{4}}\left(\frac{F^{\prime}}{2} \mathcal{M}_{d}^{\prime}-\mathcal{M}_{d}\right) \tilde{\otimes}\left(\frac{F^{\prime}}{2} \mathcal{M}_{d}^{\dagger \prime}-\mathcal{M}_{d}^{\dagger}\right)+\frac{1}{6 v^{4}} \mathcal{M}_{d}^{\prime \prime} \tilde{\otimes} \mathcal{M}_{d}^{\dagger \prime \prime}-\frac{1}{3 v^{4}}\left(F^{-1 / 2} \mathcal{M}_{d}\right)^{\prime} \tilde{\otimes}\left(F^{-1 / 2} \mathcal{M}_{d}^{\dagger}\right)^{\prime}, \\
\Gamma_{L R 13}= & \frac{3 F^{-2}}{v^{4}}\left(\frac{F^{\prime}}{2} \mathcal{M}_{d}^{\prime}-\mathcal{M}_{d}\right) \tilde{\otimes}\left(\frac{F^{\prime}}{2} \mathcal{M}_{d}^{\dagger \prime}-\mathcal{M}_{d}^{\dagger}\right)+\frac{1}{v^{4}} \mathcal{M}_{d}^{\prime \prime} \tilde{\otimes} \mathcal{M}_{d}^{\dagger \prime \prime}-\frac{2}{v^{4}}\left(F^{-1 / 2} \mathcal{M}_{d}\right)^{\prime} \tilde{\otimes}\left(F^{-1 / 2} \mathcal{M}_{d}^{\dagger}\right)^{\prime}, \\
\Gamma_{L R 17}= & \frac{3 F^{-2}}{2 v^{4}}\left(\frac{F^{\prime}}{2} \mathcal{M}_{e}^{\prime}-\mathcal{M}_{e}\right) \tilde{\otimes}\left(\frac{F^{\prime}}{2} \mathcal{M}_{e}^{\dagger \prime}-\mathcal{M}_{e}^{\dagger}\right)+\frac{1}{2 v^{4}} \mathcal{M}_{e}^{\prime \prime} \tilde{\otimes} \mathcal{M}_{e}^{\dagger \prime \prime}-\frac{1}{v^{4}}\left(F^{-1 / 2} \mathcal{M}_{e}\right)^{\prime} \tilde{\otimes}\left(F^{-1 / 2} \mathcal{M}_{e}^{\dagger}\right)^{\prime}, \\
\Gamma_{L R 18}= & \frac{3 F^{-2}}{2 v^{4}}\left(\frac{F^{\prime}}{2} \mathcal{M}_{d}^{\prime}-\mathcal{M}_{d}\right) \tilde{\otimes}\left(\frac{F^{\prime}}{2} \mathcal{M}_{e}^{\dagger \prime}-\mathcal{M}_{e}^{\dagger}\right)+\frac{1}{2 v^{4}} \mathcal{M}_{d}^{\prime \prime} \tilde{\otimes} \mathcal{M}_{e}^{\dagger \prime \prime}-\frac{1}{v^{4}}\left(F^{-1 / 2} \mathcal{M}_{d}\right)^{\prime} \tilde{\otimes}\left(F^{-1 / 2} \mathcal{M}_{e}^{\dagger}\right)^{\prime} .
\end{aligned}
$$

\section{F. Renormalization group equations for the coefficients of $\mathcal{L}_{4}$}

In the preceding subsections we obtained the counterterms of the next-to-leading order Lagrangian $\mathcal{L}_{4}$, employing a systematic decomposition into basis operators. We are now in a position to derive the renormalization group equations for the operator coefficients.

Summarizing the previous results, we may write

$\mathcal{L}_{4}=\sum_{i} \stackrel{\circ}{\mathcal{O}}_{i} \stackrel{\circ}{F}_{i}=\sum_{i} \mathcal{O}_{i}\left(F_{i}+\frac{\Gamma_{i}}{16 \pi^{2}} \frac{1}{d-4}\right) \mu^{d-4}$,

where the sum extends over all the NLO terms in our basis, comprising the classes $\beta_{1}, U h D^{4}, \psi^{2} U h D, \psi^{2} U h D^{2}$, and $\psi^{4} U h$. The quantities $F_{i}$ and $\Gamma_{i}$ are functions of $\eta=h / v$ and, for the fermionic terms, tensors in generation space.

The second equality in (5.48) expresses the unrenormalized coefficients $\stackrel{\circ}{F}_{i}$ through their renormalized version plus counterterms $\sim \Gamma_{i}$, written here in the MS scheme. The counterterms are equal and opposite in sign to the one-loop divergences, displayed in (5.2)-(5.6). Inspecting the latter, we note that all terms $\mathcal{O}_{i} \Gamma_{i}$ have canonical dimension exactly 4 , in $d=4-2 \varepsilon$ space-time dimensions; once in this context we take $g, g^{\prime}$ and $v$ to mean $g \mu^{\varepsilon}, g^{\prime} \mu^{\varepsilon}$ and $v \mu^{-\varepsilon}$, respectively. Then their dimensions are $[g]=\left[g^{\prime}\right]=\varepsilon$, $[v]=1-\varepsilon$, and $g, g^{\prime}$, and $v$ become $\mu$ independent at tree level. It follows that also $d \Gamma_{i} / d \ln \mu=0$ at tree level. Since $\left[\mathcal{L}_{4}\right]=d,\left[\mathcal{O}_{i} \Gamma_{i}\right]=4$ implies the factor $\mu^{d-4}$ shown on the right-hand side of (5.48). From the $\mu$ independence of $\stackrel{\circ}{F}_{i}$ we then infer the renormalization group equations (in 4 space-time dimensions)

$$
16 \pi^{2} \frac{d}{d \ln \mu} F_{i}=-\Gamma_{i} .
$$

The various $\Gamma_{i}$ are given in the previous sections, for $i=\beta_{1}$ in (5.11), D1, D2, D7, D8, D11 in (5.14), (5.15), $\psi V k$ in (5.22), $\psi S k$ in (5.27), FYk in (5.45), STk in (5.46), and $L R k$ in (5.47). The one-loop beta functions vanish for all couplings not present in the preceding list.

\section{COMPARISON WITH SMEFT}

SMEFT $[25,26]$ is the effective field theory formulation of the electroweak and strong interactions, where the operators are organized as an expansion according to their canonical dimension. To lowest order (dimension 4), it coincides with the SM. Excluding lepton-number violating effects, the leading corrections are given by operators of dimension 6.

Even though SMEFT is organized differently than the EWChL, there is an overlap [27], which can be used as a cross-check of our calculation. The one-loop renormalization of the SM at dimension 4 has already been shown in [16] to follow as a special case from the renormalization of the EWChL. Beyond that, the one-loop divergences of the EWChL discussed here also contain the renormalization of those dimension- 6 terms in SMEFT that have chiral dimension 2 and are thus contained in (2.1). In the SMEFT basis of [26], these terms can be expressed as

$$
\Delta \mathcal{L}_{2, d=6}=\frac{1}{\Lambda^{2}}\left(C_{\phi \square} Q_{\phi \square}+C_{\phi} Q_{\phi}+C_{\psi \phi}^{r s} Q_{\psi \phi}^{r s}\right),
$$

where

$$
\begin{gathered}
Q_{\phi \square}=\phi^{\dagger} \phi \square \phi^{\dagger} \phi, \\
Q_{\phi}=\left(\phi^{\dagger} \phi\right)^{3},
\end{gathered}
$$




$$
Q_{\psi \phi}^{r s}=\phi^{\dagger} \phi\left(\bar{\psi}_{L}(\tilde{\phi}, \phi)\right)^{r} \psi_{R}^{s} .
$$

Here $r, s$ are fermion flavor indices and $\phi$ is the Higgs doublet.

By extracting the corresponding terms from the one-loop divergences of the EWChL, one should obtain the known one-loop renormalization of SMEFT [28-31], coming from the single insertion of the dimension- 6 operators (6.2)-(6.4). In order to do this, we need the relations between the nonlinear and the linear representation of the Higgs sector, in particular,

$$
\begin{aligned}
(\tilde{\phi}, \phi) & =\frac{v}{\sqrt{2}}(1+\eta) U, \quad \phi^{\dagger} \phi=\frac{v^{2}}{2}(1+\eta)^{2}, \\
D^{\mu} \phi^{\dagger} D_{\mu} \phi & =\frac{1}{2} \partial^{\mu} h \partial_{\mu} h+\frac{v^{2}}{4}\left\langle L^{\mu} L_{\mu}\right\rangle(1+\eta)^{2},
\end{aligned}
$$

where $\phi$ is the complex Higgs doublet in the conventional linear representation, and $\tilde{\phi}_{i}=\epsilon_{i j} \phi_{j}^{*}$.

The operators in (6.2)-(6.4) modify the $\eta$-dependent functions $F, V$, and $\mathcal{M}$ from their SM form

$$
\begin{aligned}
F_{\mathrm{SM}} & =(1+\eta)^{2}, \\
V_{\mathrm{SM}} & =-\frac{m^{2} v^{2}}{2}(1+\eta)^{2}+\frac{\lambda v^{4}}{8}(1+\eta)^{4}, \\
\mathcal{M}_{\mathrm{SM}} & =\frac{v}{\sqrt{2}} \mathcal{Y}(1+\eta),
\end{aligned}
$$

where $\mathcal{Y}=\operatorname{diag}\left(\mathcal{Y}_{u}, \mathcal{Y}_{d}, \mathcal{Y}_{\nu}, \mathcal{Y}_{e}\right)$ collects the Yukawa matrices of the SM.

The simplest case is the renormalization of $C_{\phi} Q_{\phi}$. Here $F$ and $\mathcal{M}$ take their SM values, while the potential becomes $V=V_{\mathrm{SM}}+\Delta V$, with

$$
\Delta V=-\frac{C_{\phi}}{\Lambda^{2}} \frac{v^{6}}{8}(1+\eta)^{6} .
$$

This term only affects the contribution $\mathcal{L}_{\text {div }}^{(0)}$ in $(\mathrm{C} 2)$, from which we extract the term of first order in $C_{\phi}$,

$$
\begin{aligned}
32 \pi^{2} \varepsilon \Delta \mathcal{L}_{\mathrm{div}}^{\phi}= & -\frac{C_{\phi}}{\Lambda^{2}}\left(\phi^{\dagger} \phi\right)^{3}\left[\frac{3}{2}\left(3 g^{2}+g^{\prime 2}\right)+54 \lambda\right] \\
& +24 \frac{m^{2}}{\Lambda^{2}} C_{\phi}\left(\phi^{\dagger} \phi\right)^{2} .
\end{aligned}
$$

As discussed e.g., in [32], we still need to subtract $K_{(\phi)}=$ $6\left(3 g^{2}+g^{\prime 2}-\left\langle\left\langle\mathcal{Y}^{\dagger} \mathcal{Y}\right\rangle\right\rangle\right)$ from the term in square brackets, due to the renormalization of $\phi$ inside $Q_{\phi}$. We then find

$$
\begin{aligned}
& \beta_{\phi} \supseteq\left[-\frac{9}{2}\left(3 g^{2}+g^{2}\right)+54 \lambda+6\left\langle\left\langle\mathcal{Y}^{\dagger} \mathcal{Y}\right\rangle\right\rangle\right] C_{\phi}, \\
& \beta_{\lambda} \supseteq 48 \frac{m^{2}}{\Lambda^{2}} C_{\phi}
\end{aligned}
$$

for the contribution of $C_{\phi}$ to the beta functions of SMEFT at dimension 4 and 6, in agreement with [28-31] and the compilation in [33]. We recall that the beta functions of coefficient $C_{i}$ are defined as $\beta_{i}=16 \pi^{2} d C_{i} / d \ln \mu$ and the operator multiplying $\lambda$ is $Q_{\lambda}^{(4)}=-\left(\phi^{\dagger} \phi\right)^{2} / 2$ [32].

We next consider the renormalization of the modified Yukawa term $C_{\psi \phi}^{r s} Q_{\psi \phi}^{r s}$. In this case, $F$ and $V$ are the same as in the SM, but $\mathcal{M}=\mathcal{M}_{\mathrm{SM}}+\Delta \mathcal{M}$, with

$$
\Delta \mathcal{M}=-\frac{v^{3}}{2 \sqrt{2} \Lambda^{2}}(1+\eta)^{3} C_{\psi \phi}
$$

Here $C_{\psi \phi}=\operatorname{diag}\left(C_{u \phi}, C_{d \phi}, C_{\nu \phi}, C_{e \phi}\right)$, where the entries are matrices in generation space. Working out the terms to first order in $\Delta \mathcal{M}$ from $\mathcal{L}_{\text {div }}$ in (3.1), and expressing the result through the Higgs-doublet $\phi$, we obtain

$$
\begin{aligned}
32 \pi^{2} \varepsilon \Lambda^{2} \Delta \mathcal{L}_{\mathrm{div}}^{\psi \phi}= & 4 \eta_{1} m^{2}\left(\phi^{\dagger} \phi\right)^{2}+4\left(-\eta_{1} \lambda+\left\langle\left\langle C_{\psi \phi}^{\dagger} \mathcal{Y} \mathcal{Y}^{\dagger} \mathcal{Y}+\text { H.c. }\right\rangle\right)\left(\phi^{\dagger} \phi\right)^{3}\right. \\
& +\left\{6 m^{2} \bar{\psi}_{L}(\tilde{\phi}, \phi) C_{\psi \phi} \psi_{R}-2 \eta_{1} \phi^{\dagger} \phi \bar{\psi}_{L}(\tilde{\phi}, \phi) \mathcal{Y} \psi_{R}+4 i \eta_{5} \phi^{\dagger} \phi \bar{\psi}_{L}(\tilde{\phi}, \phi) \mathcal{Y} T_{3} \psi_{R}\right. \\
& -\phi^{\dagger} \phi \bar{\psi}_{L}(\tilde{\phi}, \phi)\left[-6 C_{F} g_{s}^{2} C_{q \phi}+\left(\frac{3}{4}\left(3 g^{2}+g^{\prime 2}\right)-6 Y_{L} Y_{R} g^{2}+12 \lambda\right) C_{\psi \phi}\right] \psi_{R} \\
& \left.-\phi^{\dagger} \phi \bar{\psi}_{L}(\tilde{\phi}, \phi)\left[7 \mathcal{Y} \mathcal{Y}^{\dagger} C_{\psi \phi}+6 C_{\psi \phi} \mathcal{Y}^{\dagger} \mathcal{Y}+2 \mathcal{Y} C_{\psi \phi}^{\dagger} \mathcal{Y}-\left\langle C_{\psi \phi} \mathcal{Y}^{\dagger}\right\rangle \mathcal{Y}-2\left\langle\mathcal{Y} C_{\psi \phi}^{\dagger}\right\rangle \mathcal{Y}-\frac{3}{2}\left\langle\mathcal{Y} \mathcal{Y}^{\dagger}\right\rangle C_{\psi \phi}\right] \psi_{R}+\text { H.c. }\right\},
\end{aligned}
$$

where we defined $C_{q \phi}=\operatorname{diag}\left(C_{u \phi}, C_{d \phi}, 0,0\right)$ and [33]

$$
\eta_{1} \equiv \frac{1}{2}\left\langle\left\langle C_{\psi \phi}^{\dagger} \mathcal{Y}+\mathcal{Y}^{\dagger} C_{\psi \phi}\right\rangle\right\rangle, \quad i \eta_{5} \equiv\left\langle\left\langle\left(C_{\psi \phi}^{\dagger} \mathcal{Y}-\mathcal{Y}^{\dagger} C_{\psi \phi}\right) T_{3}\right\rangle\right\rangle
$$


Since the EWChL is formulated explicitly in the broken phase, in contrast to SMEFT, terms vanishing as $v \rightarrow 0$ after expressing all scalar fields through the doublet $\phi$ have to be omitted in deriving (6.11). The beta-function contributions proportional to $C_{\psi \phi}$ can be read off from (6.11), once the field renormalization factor $K_{(\psi \phi)}=3\left(3 g^{2}+\right.$ $\left.g^{\prime 2}-\left\langle\left\langle\mathcal{Y}^{\dagger} \mathcal{Y}\right\rangle\right\rangle\right)$ has been subtracted from the coefficient of $-C_{\psi \phi}^{r s} Q_{\psi \phi}^{r s}$. We find the entries

$$
\begin{aligned}
& \beta_{\lambda} \supseteq 8 \eta_{1} \frac{m^{2}}{\Lambda^{2}}, \quad \beta_{\mathcal{Y}} \supseteq 6 \frac{m^{2}}{\Lambda^{2}} C_{\psi \phi}, \\
& \beta_{\phi} \supseteq 4 \eta_{1} \lambda-4\left\langle\left\langle C_{\psi \phi}^{\dagger} \mathcal{Y} \mathcal{Y}^{\dagger} \mathcal{Y}+\text { H.c. }\right\rangle\right\rangle, \\
& \beta_{\psi \phi} \supseteq 2 \eta_{1} \mathcal{Y}-4 i \eta_{5} \mathcal{Y} T_{3}-6 C_{F} g_{s}^{2} C_{q \phi} \\
&+\left(-\frac{9}{4}\left(3 g^{2}+g^{2}\right)-6 Y_{L} Y_{R} g^{2}+12 \lambda+3\left\langle\left\langle\mathcal{Y}^{\dagger} \mathcal{Y}\right\rangle\right\rangle\right) C_{\psi \phi} \\
&+7 \mathcal{Y} \mathcal{Y}^{\dagger} C_{\psi \phi}+6 C_{\psi \phi} \mathcal{Y}^{\dagger} \mathcal{Y}+2 \mathcal{Y} C_{\psi \phi}^{\dagger} \mathcal{Y} \\
&-\left\langle C_{\psi \phi} \mathcal{Y}^{\dagger}\right\rangle \mathcal{Y}-2\left\langle\mathcal{Y} C_{\psi \phi}^{\dagger}\right\rangle \mathcal{Y}-\frac{3}{2}\left\langle\mathcal{Y} \mathcal{Y}^{\dagger}\right\rangle C_{\psi \phi} .
\end{aligned}
$$

Finally, we extract the one-loop divergences induced by a single insertion of the operator $Q_{\phi \square}$. This case is more complicated than the previous ones, since $Q_{\phi \square}$ does not match the canonical form of the chiral Lagrangian $\mathcal{L}_{2}$ in (2.1). Employing a suitable field redefinition the desired information can nevertheless be obtained.

The operator $Q_{\phi \square}$ modifies the SM Lagrangian such that the kinetic term of $h=v \eta$ becomes

$$
\begin{aligned}
\mathcal{L}_{h} & \equiv \frac{1}{2} \partial_{\mu} h \partial^{\mu} h+\frac{C_{\phi \square}}{\Lambda^{2}} Q_{\phi \square} \\
& =\left(1-2 \frac{C_{\phi \square}}{\Lambda^{2}} v^{2}(1+\eta)^{2}\right) \frac{1}{2} \partial_{\mu} h \partial^{\mu} h=\frac{1}{2} \partial_{\mu} \tilde{h} \partial^{\mu} \tilde{h},
\end{aligned}
$$

with all other terms unchanged. In the second step, (6.2), (6.5) and an integration by parts have been used. In the last step, the canonical form of the kinetic term is recovered by means of the field redefinition

$$
\eta \doteq \tilde{\eta}+\frac{C_{\phi \square}}{\Lambda^{2}} \frac{v^{2}}{3}(1+\eta)^{3} \equiv \tilde{\eta}+\Delta \eta,
$$

to first order in $C_{\phi \square}$. The functions in (6.6) can next be expressed as

$$
\begin{aligned}
F_{\mathrm{SM}}(\eta) & =F_{\mathrm{SM}}(\tilde{\eta}+\Delta \eta) \\
& \doteq F_{\mathrm{SM}}(\tilde{\eta})+\frac{C_{\phi \square}}{\Lambda^{2}} \frac{v^{2}}{3}(1+\eta)^{3} F_{\mathrm{SM}}^{\prime}(\eta) \\
& \equiv F_{\mathrm{SM}}(\tilde{\eta})+\Delta F
\end{aligned}
$$

and similarly for $V_{\mathrm{SM}}$ and $\mathcal{M}_{\mathrm{SM}}$. The SM Lagrangian including the $Q_{\phi \square}$ term can thus be brought to the form of (2.1), now written with $\tilde{\eta}$ replacing $\eta$, and with the functions

$$
\begin{aligned}
F(\tilde{\eta}) & =F_{\mathrm{SM}}(\tilde{\eta})+\Delta F, \quad V(\tilde{\eta})=V_{\mathrm{SM}}(\tilde{\eta})+\Delta V \\
\mathcal{M}(\tilde{\eta}) & =\mathcal{M}_{\mathrm{SM}}(\tilde{\eta})+\Delta \mathcal{M} .
\end{aligned}
$$

In order to find the one-loop divergences proportional to $C_{\phi \square}$, we extract from (3.1)

$\mathcal{L}_{\text {div }}\left(F_{\mathrm{SM}}(\tilde{\eta})+\Delta F, \quad V_{\mathrm{SM}}(\tilde{\eta})+\Delta V, \quad \mathcal{M}_{\mathrm{SM}}(\tilde{\eta})+\Delta \mathcal{M}\right)$

the terms to first order in $\Delta F, \Delta V$ and $\Delta \mathcal{M}$. In addition, we need to reexpress the SM limit of the divergences through the original field $\eta$, which introduces a further contribution

$$
\mathcal{L}_{\mathrm{div}}^{\mathrm{SM}}(\tilde{\eta})=\mathcal{L}_{\mathrm{div}}^{\mathrm{SM}}(\eta-\Delta \eta) \doteq \mathcal{L}_{\mathrm{div}}^{\mathrm{SM}}(\eta)+\mathcal{L}_{\mathrm{div}}^{\Delta \eta} .
$$

Adding all four contributions and expressing the scalar fields in terms of $\phi$, we obtain

$$
\begin{aligned}
32 \pi^{2} \varepsilon \frac{\Lambda^{2}}{C_{\phi \square}} \Delta \mathcal{L}_{\text {div }}^{\psi \phi}= & 8 m^{4} \phi^{\dagger} \phi+m^{2}\left(\frac{20}{3} g^{2}-32 \lambda\right)\left(\phi^{\dagger} \phi\right)^{2}-2 m^{2}\left(\bar{\psi}_{L}(\tilde{\phi}, \phi) \mathcal{Y} \psi_{R}+\text { H.c. }\right) \\
& +\left(40 \lambda^{2}-\frac{20}{3} g^{2} \lambda\right)\left(\phi^{\dagger} \phi\right)^{3}-\left(8 g^{2}+\frac{8}{3} g^{\prime 2}+12 \lambda\right) Q_{\phi \square}-\frac{20}{3} g^{\prime 2} Q_{\phi D} \\
& +\phi^{\dagger} \phi\left(\bar{\psi}_{L}(\tilde{\phi}, \phi)\left[\left(-\frac{10}{3} g^{2}+2 \lambda\right) \mathcal{Y}+6 \mathcal{Y} \mathcal{Y}^{\dagger} \mathcal{Y}\right] \psi_{R}+\text { H.c. }\right) \\
& +\phi^{\dagger} i \stackrel{\leftrightarrow}{D}_{\mu} \phi \bar{\psi}_{R}\left(-\frac{g^{\prime 2}}{3} Y_{R}+\mathcal{Y}^{\dagger} \mathcal{Y} \tau_{3}\right) \gamma^{\mu} \psi_{R}-2\left(\tilde{\phi}^{\dagger} i D_{\mu} \phi \bar{u}_{R} \mathcal{Y}_{u}^{\dagger} \mathcal{Y}_{d} \gamma^{\mu} d_{R}+\text { H.c. }\right) \\
& +\phi^{\dagger} i \stackrel{\leftrightarrow}{D}_{\mu} \phi \bar{\psi}_{L}\left(-\frac{g^{\prime 2}}{3} Y_{L}-\frac{1}{2}\left\langle\mathcal{Y} \mathcal{Y}^{\dagger} \tau_{3}\right\rangle\right) \gamma^{\mu} \psi_{L}+\phi^{\dagger} i \stackrel{\leftrightarrow}{D_{\mu}} \phi \bar{\psi}_{L}\left(-\frac{g^{2}}{6}+\frac{1}{2}\left\langle\mathcal{Y} \mathcal{Y}^{\dagger}\right\rangle\right) \tau^{a} \gamma^{\mu} \psi_{L} .
\end{aligned}
$$


Here $\tau^{a} \equiv 2 T^{a}$ are the Pauli matrices, and $Q_{\phi D}=D^{\mu} \phi^{\dagger} \phi \phi^{\dagger} D_{\mu} \phi$. Subtracting the field renormalization term $K_{(\phi \square)}=12 g^{2}+4 g^{\prime 2}-4\left\langle\left\langle\mathcal{Y}^{\dagger} \mathcal{Y}\right\rangle\right\rangle$ from the bracketed term in front of $Q_{\phi \square}$, we read off the contribution of $C_{\phi \square}$ to the SMEFT beta functions:

$$
\begin{aligned}
& \beta_{m^{2}} \supseteq-8 \frac{m^{4}}{\Lambda^{2}} C_{\phi \square}, \quad \beta_{\lambda} \supseteq\left(\frac{40}{3} g^{2}-64 \lambda\right) \frac{m^{2}}{\Lambda^{2}} C_{\phi \square}, \\
& \beta_{\mathcal{Y}} \supseteq-2 \frac{m^{2}}{\Lambda^{2}} \mathcal{Y} C_{\phi \square} \\
& \beta_{\phi} \supseteq\left(\frac{20}{3} g^{2} \lambda-40 \lambda^{2}\right) C_{\phi \square}, \\
& \beta_{\phi \square} \supseteq\left(-4 g^{2}-\frac{4}{3} g^{\prime 2}+12 \lambda+4\left\langle\left\langle\mathcal{Y}^{\dagger} \mathcal{Y}\right\rangle\right) C_{\phi \square},\right. \\
& \beta_{\phi D} \supseteq \frac{20}{3} g^{\prime 2} C_{\phi \square} \\
& \beta_{\psi \phi} \supseteq\left[\left(\frac{10}{3} g^{2}-2 \lambda\right) \mathcal{Y}-6 \mathcal{Y} \mathcal{Y}^{\dagger} \mathcal{Y}\right] C_{\phi \square} \\
& \beta_{\phi \psi} \supseteq\left(\frac{g^{\prime 2}}{3} Y_{R}-\mathcal{Y}^{\dagger} \mathcal{Y} \tau_{3}\right) C_{\phi \square}, \quad \beta_{\phi u d} \supseteq 2 \mathcal{Y}_{u}^{\dagger} \mathcal{Y}_{d} C_{\phi \square} \\
& \beta_{\phi \psi}^{(1)} \supseteq\left(\frac{g^{\prime 2}}{3} Y_{L}+\frac{1}{2}\left\langle\mathcal{Y} \mathcal{Y}^{\dagger} \tau_{3}\right\rangle\right) C_{\phi \square}, \\
& \beta_{\phi \psi}^{(3)} \supseteq\left(\frac{g^{2}}{6}-\frac{1}{2}\left\langle\mathcal{Y} \mathcal{Y}^{\dagger}\right\rangle\right) C_{\phi \square} .
\end{aligned}
$$

All terms are in agreement with the known SMEFT results, as summarized e.g. in [33].

\section{BRIEF SURVEY OF RELATED LITERATURE}

Several groups computed subsets of the one-loop renormalization and RGEs of the EWChL in the past [34-39]. In our previous paper [16], we compared and found agreement with the one-loop divergences reported in [38], which treated the subset of divergences that arise from scalar loops and their corresponding beta functions. Previously, [36] had computed the scalar 1-, 2-, 3-, and 4-point functions at one loop. They focused on the scalar sector, not only regarding the particles running in the loop, but also regarding the set of operators. Since this set is not closed under the application of the equations of motion, there are ambiguities that make the RGEs listed there hard to compare with our result. In addition, the operator $\partial h \partial h F_{h}(h)$ was considered, which is always redundant [14]. However, [36] compared their results with [34] and claimed agreement in the corresponding limit.

Reference [34] and its followups [35,37] considered explicit $V V$ scattering processes and derived the RGEs for the couplings involved. These results were soon thereafter corroborated by [38]. In a different approach, [39] considered a geometric formulation of the scalar sector. They also computed the divergence structure of scalar loops and found full agreement with [38]. This means that all results previous to [16] have been cross-checked against each other, either directly or indirectly. Note that [38] also compared to the result in the Higgsless limit [40-44] and found agreement.

Shortly after we published [16], work on the same subject was described in [17]. The authors claim in the journal version of [17]: "Gauge bosons have the SM renormalization plus an extra contribution." Based on our discussion at the beginning of Sec. III A, such a statement is only meaningful once a basis of NLO operators is defined. After projecting the one-loop divergences onto our basis, we actually find that the one-loop gauge beta functions coincide with the SM ones. This result can be confirmed by looking at the SMEFT RGE (through dimension 6) in the basis of [26]. Here the only BSM contribution to the gauge-beta functions comes from $C_{\phi X} Q_{\phi X}$, an operator structure that is absent in the leading-order EWChL. The absence of new contributions to the one-loop gauge-beta functions can also be seen by comparing the SM limit with the Higgsless limit of our result. They both agree, indicating that only Goldstone-boson and gauge loops contribute to the one-loop gauge-beta functions, while the Higgs couplings (modified or not) do not. One can actually check that the contribution of Higgs one-loop diagrams does not lead to divergences, only to finite pieces.

\section{VACUUM EXPECTATION VALUE OF $h$ AND TADPOLE COUNTERTERM}

The potential $V$ for the scalar field $h$ is chosen such that $V^{\prime}(0)=0$, and hence the vev of the scalar field $h$ vanishes, $\langle h\rangle=0$ at lowest order. This property is not maintained when loop corrections are considered, and one needs to perform a finite renormalization of the potential in order to enforce it. This finite renormalization is computed here to illustrate the application of the EWChL at one loop.

Employing the background field method, we separate the various fields into a classical or background part, and a quantum, fluctuating, part. The terms involving only the background fields provide the tree-level amplitudes. This is the case, in particular for all the terms in $\mathcal{L}_{4}$. The part linear in the quantum fields does not matter, it vanishes when the classical equations of motion are enforced on the background fields, and terms with three or more quantum fields are not relevant for the computation of one-loop amplitudes. For general one-loop calculations, therefore, only terms exactly quadratic in the quantum fields, but with arbitrary powers of background fields, are required. As we are only interested in the Higgs potential here, all background fields, except for the Higgs, may be dropped in the following. Specializing to the tadpole contribution, eventually only the terms linear in the background Higgs field need to be kept. 
Hereafter, quantum fields are written with a caret, the fields without carets being background fields (except for the ghosts, which are always quantum fields). The decomposition in terms of background and quantum fields is a linear one, with the exception of the Goldstone fields, where the split is multiplicative. Omitting background fields, the Goldstone field reduces to its quantum part, for which we use

$$
\hat{U}=e^{2 i \hat{\varphi} F^{-1 / 2} / v}, \quad \hat{\varphi}=\hat{\varphi}^{a} T^{a} .
$$

Finally, one also needs to fix the gauge and add the corresponding contributions from the ghost fields. For the former, a background-gauge invariant choice [45] is made, namely ${ }^{2}$ g.f.

$$
\mathcal{L}_{\text {g.f. }}=-\frac{1}{2}\left(\partial_{\mu} \hat{B}^{\mu}+\frac{g^{\prime} v}{2} F^{1 / 2} \hat{\varphi}^{3}\right)^{2}-\left\langle\left(D^{\mu} \hat{W}_{\mu}-\frac{g v}{2} F^{1 / 2} \hat{\varphi}\right)^{2}\right\rangle .
$$

This means that the Faddeev-Popov ghost Lagrangian reads

$$
\mathcal{L}_{\text {ghosts }}=\partial^{\mu} \bar{c} \partial_{\mu} c+\frac{g^{\prime} v}{2} F \bar{c}\left(\frac{g v}{2} c^{3}-\frac{g^{\prime} v}{2} c\right)+\partial^{\mu} \bar{c}^{a} \partial_{\mu} c^{a}-\left(\frac{g v}{2}\right)^{2} F\left(\bar{c}^{1} c^{1}+\bar{c}^{2} c^{2}\right)-\frac{g v}{2} F \bar{c}^{3}\left(\frac{g v}{2} c^{3}-\frac{g^{\prime} v}{2} c\right) .
$$

In order to compute the Higgs tadpole at one loop, we first extract from $\mathcal{L}_{2}+\mathcal{L}_{\text {g.f. }}+\mathcal{L}_{\text {ghosts }}$ the contributions quadratic in all quantum fields, linear in $h$, and without any other classical field. This gives

$$
\begin{aligned}
\mathcal{L}_{2}+\mathcal{L}_{\text {g.f. }}+\mathcal{L}_{\text {ghosts }}= & -\frac{1}{4}\left(\partial_{\mu} \hat{W}_{\nu}^{a}-\partial_{\nu} \hat{W}_{\mu}^{a}\right)\left(\partial^{\mu} \hat{W}^{a \nu}-\partial^{\nu} \hat{W}^{a \mu}\right)-\frac{1}{4}\left(\partial_{\mu} \hat{B}_{\nu}-\partial_{\nu} \hat{B}_{\mu}\right)\left(\partial^{\mu} \hat{B}^{\nu}-\partial^{\nu} \hat{B}^{\mu}\right) \\
& +\frac{v^{2}}{4} F\left\langle\left(\frac{2}{v} \partial^{\mu}\left(\hat{\varphi} F^{-1 / 2}\right)+g \hat{W}^{\mu}-g^{\prime} \hat{B}^{\mu} T^{3}\right)\left(\frac{2}{v} \partial_{\mu}\left(\hat{\varphi} F^{-1 / 2}\right)+g \hat{W}_{\mu}-g^{\prime} \hat{B}_{\mu} T^{3}\right)\right\rangle \\
& +\frac{1}{2} \partial^{\mu} \hat{h} \partial_{\mu} \hat{h}-v^{2} V_{2} \hat{h}^{2}-3 v V_{3} h \hat{h}^{2}+\overline{\hat{\psi}} i \not \partial \hat{\psi}-\overline{\hat{\psi}}\left(\mathcal{M} P_{R}+\mathcal{M}^{\dagger} P_{L}\right) \hat{\psi} \\
& -\frac{1}{2}\left(\partial^{\mu} \hat{B}_{\mu}+\frac{g^{\prime} v}{2} F^{1 / 2} \hat{\varphi}^{3}\right)^{2}-\frac{1}{2}\left(\partial^{\mu} \hat{W}_{\mu}^{a}-\frac{g v}{2} F^{1 / 2} \hat{\varphi}^{a}\right)\left(\partial^{\nu} \hat{W}_{\nu}^{a}-\frac{g v}{2} F^{1 / 2} \hat{\varphi}^{a}\right) \\
& +\partial^{\mu} \bar{c} \partial_{\mu} c+\frac{g^{\prime} v}{2} F \bar{c}\left(\frac{g v}{2} c^{3}-\frac{g^{\prime} v}{2} c\right)+\partial^{\mu} \bar{c}^{a} \partial_{\mu} c^{a}-\left(\frac{g v}{2}\right)^{2} F\left(\bar{c}^{1} c^{1}+\bar{c}^{2} c^{2}\right) \\
& -\frac{g v}{2} F \bar{c}^{3}\left(\frac{g v}{2} c^{3}-\frac{g^{\prime} v}{2} c\right)+\cdots \\
= & -\frac{1}{2}\left(\partial_{\mu} \hat{W}_{\nu}^{a}\right)\left(\partial^{\mu} \hat{W}^{a \nu}\right)+\frac{F}{2}\left(\frac{g v}{2}\right)^{2} \hat{W}^{a \mu} \hat{W}_{\mu}^{a}-\frac{1}{2}\left(\partial_{\mu} \hat{B}_{\nu}\right)\left(\partial^{\mu} \hat{B}^{\nu}\right)+\frac{F}{2}\left(\frac{g^{\prime} v}{2}\right)^{2} \hat{B}^{\mu} \hat{B}_{\mu}-F \frac{g g^{\prime} v^{2}}{4} \hat{W}_{\mu}^{3} \hat{B}^{\mu} \\
& +\frac{1}{2}\left(\partial^{\mu} \hat{\varphi}^{a}\right)\left(\partial_{\mu} \hat{\varphi}^{a}\right)-\frac{F}{2}\left(\frac{g v}{2}\right)^{2} \hat{\varphi}^{a} \hat{\varphi}^{a}-\frac{F}{2}\left(\frac{g^{\prime} v}{2} \hat{\varphi}^{3}\right)^{2}-F \frac{g^{\prime} v}{2} \partial^{\mu}\left(\hat{B}_{\mu} \hat{\varphi}^{3} F^{-1 / 2}\right)+F \frac{g v}{2} \partial^{\mu}\left(\hat{W}_{\mu}^{a} \hat{\varphi}^{a} F^{-1 / 2}\right) \\
& +\frac{1}{2} \partial^{\mu} \hat{h} \partial_{\mu} \hat{h}-v^{2} V_{2} \hat{h}^{2}-3 v V_{3} h \hat{h}^{2}-\frac{1}{4 F}\left(\partial^{\mu} F\right) \partial_{\mu}\left(\hat{\varphi}^{a} \hat{\varphi}^{a}\right)+\overline{\hat{\psi}} i \not \partial \hat{\psi}-\overline{\hat{\psi}}\left(\mathcal{M} P_{R}+\mathcal{M}^{\dagger} P_{L}\right) \hat{\psi} \\
& +\partial^{\mu} \bar{c} \partial_{\mu} c+\frac{g^{\prime} v}{2} F \bar{c}\left(\frac{g v}{2} c^{3}-\frac{g^{\prime} v}{2} c\right)+\partial^{\mu} \bar{c} \partial_{\mu} c^{a}-\left(\frac{g v}{2}\right)^{2} F\left(\bar{c}^{1} c^{1}+\bar{c}^{2} c^{2}\right) \\
& -\frac{g v}{2} F^{3}\left(\frac{g v}{2} c^{3}-\frac{g^{\prime} v}{2} c\right)+\cdots \\
&
\end{aligned}
$$

\footnotetext{
${ }^{2}$ At this stage, the QCD part does not matter, and is therefore omitted for the time being.
} 


$$
\begin{aligned}
= & -\frac{1}{2}\left(\partial_{\mu} \hat{W}_{\nu}^{1}\right)\left(\partial^{\mu} \hat{W}^{1 \nu}\right)-\frac{1}{2}\left(\partial_{\mu} \hat{W}_{\nu}^{2}\right)\left(\partial^{\mu} \hat{W}^{2 \nu}\right)+\frac{F}{2}\left(\frac{g v}{2}\right)^{2}\left(\hat{W}^{1 \mu} \hat{W}_{\mu}^{1}+\hat{W}^{2 \mu} \hat{W}_{\mu}^{2}\right) \\
& -\frac{1}{2}\left(\partial_{\mu} \hat{Z}_{\nu}\right)\left(\partial^{\mu} \hat{Z}^{\nu}\right)+\frac{F}{2}\left(\frac{v}{2}\right)^{2}\left(g^{2}+g^{\prime 2}\right) \hat{Z}^{\mu} \hat{Z}_{\mu}-\frac{1}{2}\left(\partial_{\mu} \hat{A}_{\nu}\right)\left(\partial^{\mu} \hat{A}^{\nu}\right)+\frac{1}{2} \partial^{\mu} \hat{h} \partial_{\mu} \hat{h}-v^{2} V_{2} \hat{h}^{2}-3 v V_{3} h \hat{h}^{2} \\
& +\frac{1}{2}\left(\partial^{\mu} \hat{\varphi}^{1}\right)\left(\partial_{\mu} \hat{\varphi}^{1}\right)+\frac{1}{2}\left(\partial^{\mu} \hat{\varphi}^{2}\right)\left(\partial_{\mu} \hat{\varphi}^{2}\right)-\frac{F}{2}\left(\frac{g v}{2}\right)^{2}\left(\hat{\varphi}^{1} \hat{\varphi}^{1}+\hat{\varphi}^{2} \hat{\varphi}^{2}\right)+\frac{1}{2}\left(\partial^{\mu} \hat{\varphi}^{3}\right)\left(\partial_{\mu} \hat{\varphi}^{3}\right)-\frac{F}{2}\left(\frac{v}{2}\right)^{2}\left(g^{2}+g^{\prime 2}\right) \hat{\varphi}^{3} \hat{\varphi}^{3} \\
& -F \frac{g^{\prime} v}{2} \partial^{\mu}\left(\hat{B}_{\mu} \hat{\varphi}^{3} F^{-1 / 2}\right)+F \frac{g v}{2} \partial^{\mu}\left(\hat{W}_{\mu}^{a} \hat{\varphi}^{a} F^{-1 / 2}\right)+\frac{F_{1}}{4 v}\left(\hat{\varphi}^{a} \hat{\varphi}^{a}\right) \square h+\overline{\hat{\psi}} i \not \partial \hat{\psi}-\overline{\hat{\psi}}\left(\mathcal{M} P_{R}+\mathcal{M}^{\dagger} P_{L}\right) \hat{\psi} \\
& +\partial^{\mu} \bar{c}_{\gamma} \partial_{\mu} c_{\gamma}+\partial^{\mu} \bar{c}^{1} \partial_{\mu} c^{1}+\partial^{\mu} \bar{c}^{2} \partial_{\mu} c^{2}-\left(\frac{g v}{2}\right)^{2} F\left(\bar{c}^{1} c^{1}+\bar{c}^{2} c^{2}\right)+\partial^{\mu} \bar{c}_{Z} \partial_{\mu} c_{Z}-\left(\frac{v}{2}\right)^{2}\left(g^{2}+g^{\prime 2}\right) F \bar{c}_{Z} c_{Z}+\cdots
\end{aligned}
$$

In the first equality, the terms of the fourth line represent the contributions from the gauge-fixing term and the fifth and sixth lines the contributions from the corresponding Faddeev-Popov ghosts. Total derivatives have been dropped. In the last step, we have introduced mass eigenstates in the gauge sector in the usual way by defining the combinations

$$
\begin{aligned}
& A_{\mu}=\frac{g}{\sqrt{g^{2}+g^{\prime 2}}} B_{\mu}+\frac{g^{\prime}}{\sqrt{g^{2}+g^{\prime 2}}} W_{\mu}^{3}, \quad Z_{\mu}=\frac{g}{\sqrt{g^{2}+g^{\prime 2}}} W_{\mu}^{3}-\frac{g^{\prime}}{\sqrt{g^{2}+g^{\prime 2}}} B_{\mu}, \\
& c_{\gamma}=\frac{g}{\sqrt{g^{2}+g^{\prime 2}}} c+\frac{g^{\prime}}{\sqrt{g^{2}+g^{\prime 2}}} c^{3}, \quad c_{Z}=\frac{g}{\sqrt{g^{2}+g^{\prime 2}}} c^{3}-\frac{g^{\prime}}{\sqrt{g^{2}+g^{\prime 2}}} c .
\end{aligned}
$$

In addition, the last expression involves $\square h$, where $h$ is the classical field, so that one may use its equation of motion:

$$
\square h=-m_{h}^{2} h+\cdots, \quad m_{h}^{2}=2 v^{2} V_{2}
$$

Taking the background field $h$ to zero in (8.4), we obtain the kinetic terms for all the quantum fields. Those terms fix the propagators entering the calculation. In order to compute the vev of $h$ at one loop, we further need to extract from (8.4) all the cubic terms that are both linear in the background field $h$ and quadratic in the quantum fields, i.e.,

$$
\begin{aligned}
\mathcal{L}_{2}+\mathcal{L}_{\text {g.f. }}+\mathcal{L}_{\text {ghosts }}= & \cdots+\frac{h}{v} F_{1}\left[-\frac{v^{2}}{2} V_{2}\left(\hat{\varphi}^{a} \hat{\varphi}^{a}\right)-\frac{1}{2}\left(\frac{g v}{2}\right)^{2}\left(\hat{\varphi}^{1} \hat{\varphi}^{1}+\hat{\varphi}^{2} \hat{\varphi}^{2}\right)-\frac{1}{2}\left(\frac{v}{2}\right)^{2}\left(g^{2}+g^{\prime 2}\right) \hat{\varphi}^{3} \hat{\varphi}^{3}-3 v^{2} \frac{V_{3}}{F_{1}} \hat{h}^{2}\right. \\
& +\frac{1}{2}\left(\frac{g v}{2}\right)^{2}\left(\hat{W}_{\mu}^{1} \hat{W}^{1 \mu}+\hat{W}_{\mu}^{2} \hat{W}^{2 \mu}\right)+\frac{1}{2}\left(\frac{v}{2}\right)^{2}\left(g^{2}+g^{\prime 2}\right) \hat{Z}_{\mu} \hat{Z}^{\mu}-\frac{g^{\prime} v}{2} \partial^{\mu}\left(\hat{B}_{\mu} \hat{\varphi}^{3}\right)+\frac{g v}{2} \partial^{\mu}\left(\hat{W}_{\mu}^{a} \hat{\varphi}^{a}\right) \\
& \left.-\frac{1}{F_{1}} \overline{\hat{\psi}}\left(\mathcal{M}_{1} P_{R}+\mathcal{M}_{1}^{\dagger} P_{L}\right) \hat{\psi}-\left(\frac{g v}{2}\right)^{2}\left(\bar{c}^{1} c^{1}+\bar{c}^{2} c^{2}\right)-\left(\frac{v}{2}\right)^{2}\left(g^{2}+g^{\prime 2}\right) \bar{c}_{Z} c_{Z}\right]+\cdots
\end{aligned}
$$

Then one finds

$$
\begin{aligned}
\langle h\rangle_{\text {loop }}= & \frac{1}{2 v^{2} V_{2}} \times \frac{i F_{1}}{2 v} \int \frac{d^{d} q}{(2 \pi)^{d}}\left[2 \frac{-v^{2} V_{2}-(g v / 2)^{2}}{q^{2}-(g v / 2)^{2}}+\frac{-v^{2} V_{2}-v^{2}\left(g^{2}+g^{\prime 2}\right) / 4}{q^{2}-v^{2}\left(g^{2}+g^{\prime 2}\right) / 4}-6 v^{2} \frac{V_{3}}{F_{1}} \frac{1}{q^{2}-2 v^{2} V_{2}}\right. \\
& +d \times 2\left(\frac{g v}{2}\right)^{2} \frac{-1}{q^{2}-(g v / 2)^{2}}+d \times\left(\frac{v}{2}\right)^{2}\left(g^{2}+g^{\prime 2}\right) \frac{-1}{q^{2}-v^{2}\left(g^{2}+g^{\prime 2}\right) / 4} \\
& -4(-1)\left(\frac{g v}{2}\right)^{2} \frac{1}{q^{2}-(g v / 2)^{2}}-2(-1)\left(\frac{v}{2}\right)^{2}\left(g^{2}+g^{\prime 2}\right) \frac{1}{q^{2}-v^{2}\left(g^{2}+g^{\prime 2}\right) / 4} \\
& \left.-\frac{2}{F_{1}}(-1) \operatorname{tr}\left[\left(\mathcal{M}_{1} P_{R}+\mathcal{M}_{1}^{\dagger} P_{L}\right)\left(\not 1+\mathcal{M}_{0} P_{L}+\mathcal{M}_{0}^{\dagger} P_{R}\right)\left(\frac{1}{q^{2}-\mathcal{M}_{0} \mathcal{M}_{0}^{\dagger}} P_{R}+\frac{1}{q^{2}-\mathcal{M}_{0}^{\dagger} \mathcal{M}_{0}} P_{L}\right)\right]\right]
\end{aligned}
$$




$$
\begin{aligned}
= & \frac{i}{2 v^{2} V_{2}} \times \frac{F_{1}}{2 v}\left[2(1-d)\left(\frac{g v}{2}\right)^{2} A\left(\frac{g^{2} v^{2}}{4}\right)+(1-d)\left(\frac{v}{2}\right)^{2}\left(g^{2}+g^{\prime 2}\right) A\left(\frac{v^{2}}{4}\left(g^{2}+g^{\prime 2}\right)\right)\right. \\
& -2 v^{2} V_{2} A\left(\frac{g^{2} v^{2}}{4}\right)-v^{2} V_{2} A\left(\frac{v^{2}}{4}\left(g^{2}+g^{\prime 2}\right)\right)-6 v^{2} \frac{V_{3}}{F_{1}} A\left(2 v^{2} V_{2}\right) \\
& \left.+\frac{4}{F_{1}}\left\langle\left\langle\mathcal{M}_{1} \mathcal{M}_{0}^{\dagger} A\left(\mathcal{M}_{0} \mathcal{M}_{0}^{\dagger}\right)+\mathcal{M}_{1}^{\dagger} \mathcal{M}_{0} A\left(\mathcal{M}_{0}^{\dagger} \mathcal{M}_{0}\right)\right\rangle\right\rangle\right] .
\end{aligned}
$$

The first equality shows, successively, the contributions from the scalar fields (first line), from the gauge fields (second line), from the ghosts (third line), and from the fermion fields (fourth line). The second equality involves the dimensionally regularized one-point loop function

$$
A\left(m^{2}\right)=\int \frac{d^{4} q}{(2 \pi)^{4}} \frac{1}{q^{2}-m^{2}} \rightarrow \mu^{4-d} \int \frac{d^{d} q}{(2 \pi)^{d}} \frac{1}{q^{2}-m^{2}}=-i m^{2} \frac{1}{16 \pi^{2}}\left[\frac{2}{d-4}-\ln 4 \pi+\gamma_{E}+\ln \frac{m^{2}}{\mu^{2}}-1\right] .
$$

On the other hand, in the $\overline{\mathrm{MS}}$ scheme, the counterterm $\Delta V=V^{\prime} \delta$ in (4.12) contributes to $\langle h\rangle$ as

$$
\begin{aligned}
\langle h\rangle_{\Delta V} \equiv & \left.\frac{1}{2 v^{2} V_{2}} \frac{\delta(-\Delta V)}{\delta h}\right|_{h=0} \\
= & \frac{1}{2 v^{2} V_{2}} \frac{1}{16 \pi^{2}}\left[\frac{1}{d-4}-\frac{1}{2}\left(\ln 4 \pi-\gamma_{E}\right)\right]\left[\frac{3}{2}\left(3 g^{4}+2 g^{2} g^{\prime 2}+g^{\prime 4}\right) \frac{v^{3}}{8} F_{1}+\left(3 g^{2}+g^{\prime 2}\right) \frac{v^{3}}{4} F_{1} V_{2}\right. \\
& \left.+12 v^{3} V_{2} V_{3}-\frac{4}{v}\left\langle\left\langle\mathcal{M}_{0}^{\dagger} \mathcal{M}_{0}\left(\mathcal{M}_{1}^{\dagger} \mathcal{M}_{0}+\mathcal{M}_{0}^{\dagger} \mathcal{M}_{1}\right)\right\rangle\right\rangle\right] .
\end{aligned}
$$

One thus obtains the finite, but scale-dependent, result

$$
\begin{aligned}
\langle h\rangle_{\text {loop }}+\langle h\rangle_{\Delta V}= & \frac{1}{2 v^{2} V_{2}} \times \frac{1}{16 \pi^{2}}\left[-\frac{3 v^{3} F_{1}}{16} g^{4}\left(\ln \frac{g^{2} v^{2}}{4 \mu^{2}}-\frac{1}{3}\right)-\frac{3 v^{3} F_{1}}{32}\left(g^{2}+g^{\prime 2}\right)^{2}\left(\ln \frac{\left(g^{2}+g^{\prime 2}\right) v^{2}}{4 \mu^{2}}-\frac{1}{3}\right)\right. \\
& -\frac{v^{3}}{8} F_{1} V_{2}\left(2 g^{2}\left(\ln \frac{g^{2} v^{2}}{4 \mu^{2}}-1\right)+\left(g^{2}+g^{\prime 2}\right)\left(\ln \frac{\left(g^{2}+g^{\prime 2}\right) v^{2}}{4 \mu^{2}}-1\right)\right)-6 v^{3} V_{2} V_{3}\left(\ln \frac{2 v^{2} V_{2}}{\mu^{2}}-1\right) \\
& +\frac{2}{v}\left\langle\left\langle\mathcal{M}_{1} \mathcal{M}_{0}^{\dagger} \mathcal{M}_{0} \mathcal{M}_{0}^{\dagger}\left(\ln \frac{\mathcal{M}_{0} \mathcal{M}_{0}^{\dagger}}{\mu^{2}}-1\right)+\mathcal{M}_{1}^{\dagger} \mathcal{M}_{0} \mathcal{M}_{0}^{\dagger} \mathcal{M}_{0}\left(\ln \frac{\mathcal{M}_{0}^{\dagger} \mathcal{M}_{0}}{\mu^{2}}-1\right)\right\rangle\right],
\end{aligned}
$$

where $(d-1) A\left(m^{2}\right)=3 A\left(m^{2}\right)-i m^{2} /\left(8 \pi^{2}\right)$ has been used.

In order to ensure that $V^{\prime}(0)$ vanishes at the one-loop level, one thus must add to $\Delta V$ a tadpole part, $\Delta V \rightarrow \Delta V+h T(\mu)$, with $T(\mu)$ chosen such that $\langle h\rangle_{\text {loop }}+\langle h\rangle_{\Delta V}+\langle h\rangle_{T}=0$. This requires

$$
\begin{aligned}
T(\mu)= & -\frac{1}{16 \pi^{2}}\left[\frac{3 v^{3} F_{1}}{16} g^{4}\left(\ln \frac{g^{2} v^{2}}{4 \mu^{2}}-\frac{1}{3}\right)+\frac{3 v^{3} F_{1}}{32}\left(g^{2}+g^{\prime 2}\right)^{2}\left(\ln \frac{\left(g^{2}+g^{\prime 2}\right) v^{2}}{4 \mu^{2}}-\frac{1}{3}\right)\right. \\
& +\frac{v^{3}}{8} F_{1} V_{2}\left(2 g^{2}\left(\ln \frac{g^{2} v^{2}}{4 \mu^{2}}-1\right)+\left(g^{2}+g^{\prime 2}\right)\left(\ln \frac{\left(g^{2}+g^{\prime 2}\right) v^{2}}{4 \mu^{2}}-1\right)\right)+6 v^{3} V_{2} V_{3}\left(\ln \frac{2 v^{2} V_{2}}{\mu^{2}}-1\right) \\
& -\frac{2}{v}\left\langle\left\langle\mathcal{M}_{1} \mathcal{M}_{0}^{\dagger} \mathcal{M}_{0} \mathcal{M}_{0}^{\dagger}\left(\ln \frac{\mathcal{M}_{0} \mathcal{M}_{0}^{\dagger}}{\mu^{2}}-1\right)+\mathcal{M}_{1}^{\dagger} \mathcal{M}_{0} \mathcal{M}_{0}^{\dagger} \mathcal{M}_{0}\left(\ln \frac{\mathcal{M}_{0}^{\dagger} \mathcal{M}_{0}}{\mu^{2}}-1\right)\right\rangle\right] .
\end{aligned}
$$

We note that the tadpole term $T(\mu)$ corresponds to a finite shift of the Higgs field, which can be expressed as a finite contribution $\delta_{\text {fin }}$ to the divergent parameter $\delta$ introduced in (4.12),

$$
\delta \rightarrow \delta+\delta_{\text {fin }}, \quad \delta_{\text {fin }} \equiv \frac{1}{2 v^{3} V_{2}} T(\mu)
$$


According to (4.12) this leads to finite corrections in the effective Lagrangian, which cancel the Higgs tadpole in all amplitudes to one-loop order. We recall that the explicit expression for $T$ derived here refers to the $\overline{\mathrm{MS}}$ scheme. For a different choice of renormalization scheme, it would change by a finite amount.

\section{CONCLUSIONS}

In this paper we have worked out the one-loop renormalization group equations of the EWChL, taking as starting point the one-loop divergences given in [16]. The transition between the divergent structures of the theory and its beta functions is most conveniently done if the results are projected onto a complete basis. In this paper we have used the conventions adopted in [14] and worked out in detail the one-loop renormalization of both the leadingorder and next-to-leading order EWChL. Besides the complete list of the beta functions, we provide, for completeness, the explicit calculation of the finite piece needed to enforce the no-tadpole condition at one loop in the $\overline{\mathrm{MS}}$ scheme.

We point out that as a by-product of our NLO-basis choice the one-loop beta functions of the gauge couplings happen to be unaffected by potential deviations of the Higgs couplings with respect to their SM values. Actually, the one-loop renormalization of the entire gauge sector (operators with field-strength factors) is SM-like [see (3.9)]. In order to reach this conclusion in a transparent way, it is crucial to use the NLO basis in Appendix B and to reduce the divergent operators to this minimal, nonredundant set. Only after this step is done it is possible to directly read off the gauge-beta functions from the divergent piece in front of the kinetic terms without doing an additional calculation.

Our results have been cross-checked in a number of ways. All the renormalization group equations correctly reduce to the SM ones in the appropriate limit. The oneloop renormalization of SMEFT has also been used for comparison. The EWChL and SMEFT are different electroweak EFTs but their one-loop divergences partly overlap. We have explicitly shown that our results are consistent in this overlapping sector. Finally, the fact that the explicit computation of the full Higgs-tadpole at one loop is indeed rendered finite by the tadpole counterterm in our general analysis of EWChL renormalization, is yet another meaningful cross-check.

The computation presented in this paper is of relevance for the analyses of Higgs interactions at the LHC. The EWChL is the right tool to implement consistently the $\kappa$ formalism into an EFT language. The one-loop renormalization presented in this paper is necessary if one wants to extend the $\kappa$ formalism to study differential distributions in Higgs processes. Various processes of interest will be considered in the future. The framework is now available to extend their treatment to one loop.

\section{ACKNOWLEDGMENTS}

The work of G. B. has been supported in part by the Deutsche Forschungsgemeinschaft (DFG, German Research Foundation) under grant BU 1391/2-2 (Project No. 261324988) and by the DFG under Germany's Excellence Strategy EXC-2094-390783311. The work of O. C. has been supported in part by the Bundesministerium for Bildung und Forschung (BMBF FSP-105) and by the Deutsche Forschungsgemeinschaft (DFG, German Research Foundation) under Grants No. FOR 1873 and No. 396021762-TRR 257 "Particle Physics Phenomenology after the Higgs Discovery." The work of M. K. has received partial support from the OCEVU Labex (ANR-11-LABX-0060) and the A*MIDEX project (ANR11-IDEX-0001-02) funded by the "Investissements d'Avenir" French government program managed by the ANR. C. K. is supported by the Alexander von Humboldt Foundation, by the Spanish Government and ERDF funds from the EU Commission (Grants No. FPA2017-84445-P and No. SEV-2014-0398), and Grant No. DE-SC0010008 from the U.S. Department of Energy. This manuscript has been authored by Fermi Research Alliance, LLC under Contract No. DE-AC02-07CH11359 with the U.S. Department of Energy, Office of Science, Office of High Energy Physics. For cross-checks of our calculations, the programs FEYNCALC [46,47] and Mathematica [48] proved useful, as well as the compilation of formulas in [49].

\section{APPENDIX A: EQUATIONS OF MOTION}

The equations of motion (eom), derived from the leading-order Lagrangian in (2.1), are needed in particular to reduce NLO terms to a set of basis operators (see Appendix B). Here we collect the eom for the gauge fields $B_{\mu}, W_{\mu}, G_{\mu}$, the scalars $\eta=h / v, \varphi$, and the fermions $\psi_{L, R}$ :

$$
\begin{gathered}
\partial^{\mu} B_{\mu \nu}=g^{\prime}\left[\bar{\psi}_{L} \gamma_{\nu} Y_{L} \psi_{L}+\bar{\psi}_{R} \gamma_{\nu} Y_{R} \psi_{R}+\frac{v^{2}}{2} F\left\langle\tau_{L} L_{\nu}\right\rangle\right], \\
D^{\mu} W_{\mu \nu}^{a}=g\left[\bar{\psi}_{L} \gamma_{\nu} T^{a} \psi_{L}-\frac{v^{2}}{2} F\left\langle T^{a} L_{\nu}\right\rangle\right], \\
D^{\mu} G_{\mu \nu}^{A}=g_{s} \bar{q} \gamma_{\nu} T^{A} q \\
v^{2} \square \eta=-V^{\prime}+\frac{v^{2}}{4}\left\langle L_{\mu} L^{\mu}\right\rangle F^{\prime}-\bar{\psi} m^{\prime} \psi \\
F D^{\mu} L_{\mu}=-F^{\prime} \partial^{\mu} \eta L_{\mu}-\frac{4}{v^{2}} U T^{a} U^{\dagger}\left(\bar{\psi}_{L} U i T^{a} \mathcal{M} \psi_{R}+\right.\text { H.c.) }
\end{gathered}
$$

$$
\begin{aligned}
i \not D \psi_{L} & =U \mathcal{M} \psi_{R}, \\
i \not D \psi_{R} & =\mathcal{M}^{\dagger} U^{\dagger} \psi_{L} .
\end{aligned}
$$




\section{APPENDIX B: BASIS OF NLO OPERATORS}

In this section we list a basis of NLO operators for the EWChL, following $[14,15]$. The NLO terms are the independent operators of chiral dimension 4, with the field content and the symmetries of the Lagrangian in (2.1).

In the following we will assume that custodial symmetry breaking takes place at the electroweak scale. This means that the spurions of custodial symmetry breaking $\sim \tau_{L}$ carry chiral dimension. Accordingly, terms of chiral dimension 4 with extra factors of $\tau_{L}$, which were kept in the general analysis of NLO operators in [14], will be omitted. We further assume that tensor currents, e.g., $\bar{q} \sigma_{\mu \nu} U r$, only arise with a chiral dimension larger than 2. This eliminates operators with tensor currents in [14] from the list of NLO terms to be considered here.

The NLO operators can then be divided into the classes $U h D^{2}, U h D^{4}, X^{2} h, X U h D^{2}, \psi^{2} U h D, \psi^{2} U h D^{2}$, and $\psi^{4} U h$. A well-defined subset of these operators represents the counterterms necessary to renormalize the one-loop divergences of the EWChL calculated in this paper.

All operators arising at chiral dimension 4 in the classes $U h D^{2}, U h D^{4}, X^{2} h, X U h D^{2}, \psi^{2} U h D$, and $\psi^{2} U h D^{2}$ are listed below. All the operators in the classes $U h D^{2}, U h D^{4}$, $\psi^{2} U h D$, and $\psi^{2} U h D^{2}$ are needed as counterterms. On the other hand, no operator in class $X^{2} h$ or $X U h D^{2}$ is required to absorb one-loop divergences. Within the class $\psi^{4} U h$, we only list the operators that actually appear as (divergent) counterterms.

\section{Class $U h D^{2}$}

$$
\mathcal{O}_{\beta_{1}}=-v^{2}\left\langle\tau_{L} L_{\mu}\right\rangle\left\langle\tau_{L} L^{\mu}\right\rangle
$$

This operator has only two derivatives, but its coefficient comes with two powers of the weak coupling $g^{\prime}$, related to custodial symmetry breaking. In total, the term has chiral dimension 4 and enters at NLO in the EFT.

\section{Class $U h D^{4}$}

$$
\begin{aligned}
\mathcal{O}_{D 1} & =\left\langle L_{\mu} L^{\mu}\right\rangle^{2}, \quad \mathcal{O}_{D 2}=\left\langle L_{\mu} L_{\nu}\right\rangle\left\langle L^{\mu} L^{\nu}\right\rangle \\
\mathcal{O}_{D 7} & =\left\langle L_{\mu} L^{\mu}\right\rangle \partial_{\nu} \eta \partial^{\nu} \eta, \quad \mathcal{O}_{D 8}=\left\langle L_{\mu} L_{\nu}\right\rangle \partial^{\mu} \eta \partial^{\nu} \eta, \\
\mathcal{O}_{D 11} & =\left(\partial_{\mu} \eta \partial^{\mu} \eta\right)^{2} .
\end{aligned}
$$

All these operators are $C P$ even.

\section{Class $X^{2} h$}

The $C P$-even operators are

$$
\begin{aligned}
& \mathcal{O}_{X h 1}=g^{\prime 2} B_{\mu \nu} B^{\mu \nu} F_{X h 1}(h), \\
& \mathcal{O}_{X h 2}=g^{2}\left\langle W_{\mu \nu} W^{\mu \nu}\right\rangle F_{X h 2}(h), \\
& \mathcal{O}_{X h 3}=\frac{g_{s}^{2}}{2} G_{\mu \nu}^{\alpha} G^{\alpha \mu \nu} F_{X h 3}(h) .
\end{aligned}
$$

The $C P$-odd operators read

$$
\begin{aligned}
& \mathcal{O}_{X h 4}=g^{\prime 2} \varepsilon_{\mu \nu \lambda \rho} B^{\mu \nu} B^{\lambda \rho} F_{X h 4}(h), \\
& \mathcal{O}_{X h 5}=g^{2} \varepsilon_{\mu \nu \lambda \rho}\left\langle W^{\mu \nu} W^{\lambda \rho}\right\rangle F_{X h 5}(h), \\
& \mathcal{O}_{X h 6}=\frac{g_{s}^{2}}{2} \varepsilon^{\mu \nu \lambda \rho} G_{\mu \nu}^{\alpha} G_{\lambda \rho}^{\alpha} F_{X h 6}(h) .
\end{aligned}
$$

Here

$$
F_{X i}(h)=\sum_{n=1}^{\infty} f_{X i, n}\left(\frac{h}{v}\right)^{n} .
$$

\section{Class $X U h D^{2}$}

$C P$-even operators:

$$
\begin{aligned}
& \mathcal{O}_{X U 1}=g^{\prime} g B_{\mu \nu}\left\langle W^{\mu \nu} \tau_{L}\right\rangle\left(1+F_{X U 1}(h)\right), \\
& \mathcal{O}_{X U 7}=i g^{\prime} B_{\mu \nu}\left\langle\tau_{L}\left[L^{\mu}, L^{\nu}\right]\right\rangle F_{X U 7}(h), \\
& \mathcal{O}_{X U 8}=i g\left\langle W_{\mu \nu}\left[L^{\mu}, L^{\nu}\right]\right\rangle F_{X U 8}(h) .
\end{aligned}
$$

$C P$-odd operators:

$$
\begin{aligned}
\mathcal{O}_{X U 4} & =g^{\prime} g \varepsilon_{\mu \nu \lambda \rho}\left\langle\tau_{L} W^{\mu \nu}\right\rangle B^{\lambda \rho}\left(1+F_{X U 4}(h)\right), \\
\mathcal{O}_{X U 10} & =i g^{\prime} \varepsilon_{\mu \nu \lambda \rho} B^{\mu \nu}\left\langle\tau_{L}\left[L^{\lambda}, L^{\rho}\right]\right\rangle F_{X U 10}(h), \\
\mathcal{O}_{X U 11} & =i g \varepsilon_{\mu \nu \lambda \rho}\left\langle W^{\mu \nu}\left[L^{\lambda}, L^{\rho}\right]\right\rangle F_{X U 11}(h),
\end{aligned}
$$

with $F_{X i}(h)$ as in (B5). 


\section{Class $\psi^{2} U h D$}

$$
\begin{aligned}
& \mathcal{O}_{\psi V 1}=-\bar{q}_{L} \gamma^{\mu} q_{L}\left\langle\tau_{L} L_{\mu}\right\rangle, \quad \mathcal{O}_{\psi V 2}=-\bar{q}_{L} \gamma^{\mu} \tau_{L} q_{L}\left\langle\tau_{L} L_{\mu}\right\rangle, \\
& \mathcal{O}_{\psi V 3}=-\bar{q}_{L} \gamma^{\mu} U P_{12} U^{\dagger} q_{L}\left\langle L_{\mu} U P_{21} U^{\dagger}\right\rangle, \quad \mathcal{O}_{\psi V 4}=-\bar{u}_{R} \gamma^{\mu} u_{R}\left\langle\tau_{L} L_{\mu}\right\rangle, \\
& \mathcal{O}_{\psi V 5}=-\bar{d}_{R} \gamma^{\mu} d_{R}\left\langle\tau_{L} L_{\mu}\right\rangle, \quad \mathcal{O}_{\psi V 6}=-\bar{u}_{R} \gamma^{\mu} d_{R}\left\langle L_{\mu} U P_{21} U^{\dagger}\right\rangle, \\
& \mathcal{O}_{\psi V 7}=-\bar{l}_{L} \gamma^{\mu} l_{L}\left\langle\tau_{L} L_{\mu}\right\rangle, \quad \mathcal{O}_{\psi V 8}=-\bar{l}_{L} \gamma^{\mu} \tau_{L} l_{L}\left\langle\tau_{L} L_{\mu}\right\rangle, \\
& \mathcal{O}_{\psi V 9}=-\bar{l}_{L} \gamma^{\mu} U P_{12} U^{\dagger} l_{L}\left\langle L_{\mu} U P_{21} U^{\dagger}\right\rangle, \quad \mathcal{O}_{\psi V 10}=-\bar{e}_{R} \gamma^{\mu} e_{R}\left\langle\tau_{L} L_{\mu}\right\rangle,
\end{aligned}
$$

together with the Hermitian conjugates $\mathcal{O}_{\psi V 3}^{\dagger}, \mathcal{O}_{\psi V V}^{\dagger}$, and $\mathcal{O}_{\psi V 9}^{\dagger}$.

\section{Class $\psi^{2} U h D^{2}$}

$$
\begin{aligned}
\mathcal{O}_{\psi S 1} & =\bar{q}_{L} U P_{+} q_{R}\left\langle L_{\mu} L^{\mu}\right\rangle, \quad \mathcal{O}_{\psi S 2}=\bar{q}_{L} U P_{-} q_{R}\left\langle L_{\mu} L^{\mu}\right\rangle, \quad \mathcal{O}_{\psi S 7}=\bar{l}_{L} U P_{-} l_{R}\left\langle L_{\mu} L^{\mu}\right\rangle, \\
\mathcal{O}_{\psi S 10} & =\bar{q}_{L} U P_{+} q_{R}\left\langle\tau_{L} L_{\mu}\right\rangle \partial^{\mu} \eta, \quad \mathcal{O}_{\psi S 11}=\bar{q}_{L} U P_{-} q_{R}\left\langle\tau_{L} L_{\mu}\right\rangle \partial^{\mu} \eta, \quad \mathcal{O}_{\psi S 12}=\bar{q}_{L} U P_{12} q_{R}\left\langle U P_{21} U^{\dagger} L_{\mu}\right\rangle \partial^{\mu} \eta, \\
\mathcal{O}_{\psi S 13} & =\bar{q}_{L} U P_{21} q_{R}\left\langle U P_{12} U^{\dagger} L_{\mu}\right\rangle \partial^{\mu} \eta, \quad \mathcal{O}_{\psi S 14}=\bar{q}_{L} U P_{+} q_{R} \partial_{\mu} \eta \partial^{\mu} \eta, \quad \mathcal{O}_{\psi S 15}=\bar{q}_{L} U P_{-} q_{R} \partial_{\mu} \eta \partial^{\mu} \eta, \\
\mathcal{O}_{\psi S 16} & =\bar{l}_{L} U P_{-} l_{R}\left\langle\tau_{L} L_{\mu}\right\rangle \partial^{\mu} \eta, \quad \mathcal{O}_{\psi S 17}=\bar{l}_{L} U P_{12} l_{R}\left\langle U P_{21} U^{\dagger} L_{\mu}\right\rangle \partial^{\mu} \eta, \quad \mathcal{O}_{\psi S 18}=\bar{l}_{L} U P_{-} l_{R} \partial_{\mu} \eta \partial^{\mu} \eta .
\end{aligned}
$$

For this class, Hermitian conjugate versions have not been listed separately.

\section{Class $\psi^{4} U h$}

Here we list the four-fermion operators that are generated as one-loop counterterms of the EWChL. The complete basis can be found in [24]. Note that some of the $S T$-type operators originally listed there are redundant, as pointed out in Appendix A.4 of [50]. This redundancy does not affect the terms ST5, ST6, ST9, ST10 appearing below. Generation indices are suppressed. $T^{A}$ denotes the generators of color $S U(3)$.

$$
\begin{aligned}
& \mathcal{O}_{L R 1}=\bar{q}_{L} \gamma^{\mu} q_{L} \bar{u}_{R} \gamma_{\mu} u_{R}, \quad \mathcal{O}_{L R 2}=\bar{q}_{L} \gamma^{\mu} T^{A} q_{L} \bar{u}_{R} \gamma_{\mu} T^{A} u_{R}, \\
& \mathcal{O}_{L R 3}=\bar{q}_{L} \gamma^{\mu} q_{L} \bar{d}_{R} \gamma_{\mu} d_{R}, \quad \mathcal{O}_{L R 4}=\bar{q}_{L} \gamma^{\mu} T^{A} q_{L} \bar{d}_{R} \gamma_{\mu} T^{A} d_{R}, \\
& \mathcal{O}_{L R 8}=\bar{l}_{L} \gamma^{\mu} l_{L} \bar{e}_{R} \gamma_{\mu} e_{R}, \quad \mathcal{O}_{L R 9}=\bar{q}_{L} \gamma^{\mu} l_{L} \bar{e}_{R} \gamma_{\mu} d_{R}, \\
& \mathcal{O}_{L R 10}=\bar{q}_{L} \gamma^{\mu} U T_{3} U^{\dagger} q_{L} \bar{u}_{R} \gamma_{\mu} u_{R}, \quad \mathcal{O}_{L R 11}=\bar{q}_{L} \gamma^{\mu} T^{A} U T_{3} U^{\dagger} q_{L} \bar{u}_{R} \gamma_{\mu} T^{A} u_{R}, \\
& \mathcal{O}_{L R 12}=\bar{q}_{L} \gamma^{\mu} U T_{3} U^{\dagger} q_{L} \bar{d}_{R} \gamma_{\mu} d_{R}, \quad \mathcal{O}_{L R 13}=\bar{q}_{L} \gamma^{\mu} T^{A} U T_{3} U^{\dagger} q_{L} \bar{d}_{R} \gamma_{\mu} T^{A} d_{R}, \\
& \mathcal{O}_{L R 17}=\bar{l}_{L} \gamma^{\mu} U T_{3} U^{\dagger} l_{L} \bar{e}_{R} \gamma_{\mu} e_{R}, \quad \mathcal{O}_{L R 18}=\bar{q}_{L} \gamma^{\mu} U T_{3} U^{\dagger} l_{L} \bar{e}_{R} \gamma_{\mu} d_{R}, \\
& \mathcal{O}_{S T 5}=\bar{q}_{L} U P_{+} q_{R} \bar{q}_{L} U P_{-} q_{R}, \quad \mathcal{O}_{S T 6}=\bar{q}_{L} U P_{21} q_{R} \bar{q}_{L} U P_{12} q_{R}, \\
& \mathcal{O}_{S T 9}=\bar{q}_{L} U P_{+} q_{R} \bar{l}_{L} U P_{-} l_{R}, \quad \mathcal{O}_{S T 10}=\bar{q}_{L} U P_{21} q_{R} \bar{l}_{L} U P_{12} l_{R}, \\
& \mathcal{O}_{F Y 1}=\bar{q}_{L} U P_{+} q_{R} \bar{q}_{L} U P_{+} q_{R}, \quad \mathcal{O}_{F Y 3}=\bar{q}_{L} U P_{-} q_{R} \bar{q}_{L} U P_{-} q_{R}, \\
& \mathcal{O}_{F Y 5}=\bar{q}_{L} U P_{-} q_{R} \bar{q}_{R} P_{+} U^{\dagger} q_{L}, \quad \mathcal{O}_{F Y 7}=\bar{q}_{L} U P_{-} q_{R} \bar{l}_{L} U P_{-} l_{R}, \\
& \mathcal{O}_{F Y 9}=\bar{l}_{L} U P_{-} l_{R} \bar{q}_{R} P_{+} U^{\dagger} q_{L}, \quad \mathcal{O}_{F Y 10}=\bar{l}_{L} U P_{-} l_{R} \bar{l}_{L} U P_{-} l_{R} .
\end{aligned}
$$

\section{APPENDIX C: ONE-LOOP DIVERGENCES}

This Appendix gathers the explicit expressions for the complete one-loop divergences of the EWChL obtained in Ref. [16]. In terms of the decomposition introduced in Eq. (3.1), they read 


$$
\begin{aligned}
\mathcal{L}_{\text {div }}^{(1)}= & -\frac{1}{16 \pi^{2}} \frac{1}{d-4}\left\{\frac{g_{s}^{2}}{2} \frac{11 N_{c}-2 N_{f}}{3} G^{\alpha \mu \nu} G_{\mu \nu}^{\alpha}+\frac{g^{2}}{3}\left[22-\frac{\kappa^{2}+1}{4}-\left(N_{c}+1\right) N_{\mathrm{g}}\right]\left\langle W^{\mu \nu} W_{\mu \nu}\right\rangle\right. \\
& -\frac{g^{\prime 2}}{4}\left[\left(\frac{22}{27} N_{c}+2\right) N_{\mathrm{g}}+\frac{\kappa^{2}+1}{6}\right] B^{\mu \nu} B_{\mu \nu}+\frac{\kappa^{2}-1}{6} g g^{\prime}\left\langle\tau_{L} W^{\mu \nu}\right\rangle B_{\mu \nu}-\frac{\kappa^{2}-1}{12}\left(i g\left\langle W^{\mu \nu}\left[L_{\mu}, L_{\nu}\right]\right\rangle+i g^{\prime} B^{\mu \nu}\left\langle\tau_{L}\left[L_{\mu}, L_{\nu}\right]\right\rangle\right) \\
& \left.-\frac{\kappa \kappa^{\prime}}{3} \partial^{\mu} \eta\left(g\left\langle W_{\mu \nu} L^{\nu}\right\rangle-g^{\prime} B_{\mu \nu}\left\langle\tau_{L} L^{\nu}\right\rangle\right)\right\} \\
\mathcal{L}_{\text {div }}^{(0)}= & -\frac{1}{16 \pi^{2}} \frac{1}{d-4}\left\{-\frac{1}{2}\left[g^{\prime 2}\left(\kappa^{2}+3\right) \frac{v^{2} F}{4}+3 g^{2}\left(\kappa^{2}+1\right) \frac{v^{2} F}{2}+\left(\kappa^{2}-1\right) \frac{F^{\prime} V^{\prime}}{F v^{2}}-\frac{V^{\prime \prime} F}{v^{2}} \mathcal{B}-2\left\langle\left\langle\mathcal{M}^{\dagger} \mathcal{M}\right\rangle\right\rangle\right]\left\langle L^{\mu} L_{\mu}\right\rangle\right. \\
& +\frac{1}{4}\left[\left(3 g^{2}+g^{\prime 2}\right) v^{2}\left(F \mathcal{B}-4 \kappa^{2}\right)+6 \frac{F^{\prime} V^{\prime}}{F v^{2}} \mathcal{B}\right] \partial^{\mu} \eta \partial_{\mu} \eta+2\left\langle\left\langle\partial^{\mu} \mathcal{M}^{\dagger} \partial_{\mu} \mathcal{M}\right\rangle\right\rangle \\
& +\frac{3}{2}\left(3 g^{4}+2 g^{2} g^{\prime 2}+g^{\prime 4}\right) \frac{v^{4}}{16} F^{2}+\frac{3 g^{2}+g^{\prime 2}}{8} F^{\prime} V^{\prime}+\frac{3}{8}\left(\frac{F^{\prime} V^{\prime}}{F v^{2}}\right)^{2}+\frac{1}{2}\left(\frac{V^{\prime \prime}}{v^{2}}\right)^{2}-2\left\langle\left\langle(\mathcal{M} \dagger \mathcal{M})^{2}\right\rangle\right\rangle \\
& +\frac{\left(\kappa^{2}-1\right)^{2}}{6}\left\langle L_{\mu} L_{\nu}\right\rangle\left\langle L^{\mu} L^{\nu}\right\rangle+\left(\frac{\left(\kappa^{2}-1\right)^{2}}{12}+\frac{F^{2} \mathcal{B}^{2}}{8}\right)\left\langle L^{\mu} L_{\mu}\right\rangle^{2}+\frac{2}{3} \kappa^{\prime 2}\left\langle L_{\mu} L_{\nu}\right\rangle \partial^{\mu} \eta \partial^{\nu} \eta \\
& -\left(\left(\kappa^{2}-1\right) \mathcal{B}+\frac{\kappa^{\prime 2}}{6}\right)\left\langle L^{\mu} L_{\mu}\right\rangle \partial^{\nu} \eta \partial_{\nu} \eta+\frac{3}{2} \mathcal{B}^{2}\left(\partial^{\mu} \eta \partial_{\mu} \eta\right)^{2}+\frac{3}{4} g^{\prime 2} v^{2}\left(1-\kappa^{2}\right) F\left\langle\tau_{L} L^{\mu}\right\rangle\left\langle\tau_{L} L_{\mu}\right\rangle \\
& \left.\left.+4 i\left\langle\left(\partial^{\mu} \mathcal{M} \dagger \mathcal{M}-\mathcal{M}^{\dagger} \partial^{\mu} \mathcal{M}\right) T^{3}\right\rangle\right\rangle\left\langle\tau_{L} L_{\mu}\right\rangle\right\} .
\end{aligned}
$$

Here $\langle\langle\ldots\rangle\rangle$ denotes the trace over isospin, as well as generation and color indices, in distinction to $\langle\ldots\rangle$, which refers to the trace over isospin indices only.

$$
\begin{aligned}
& \mathcal{L}_{\mathrm{div}}^{(1 / 2)}=-\frac{1}{16 \pi^{2}} \frac{1}{d-4}\left\{\bar{\psi}_{L}\left(\frac{3}{2} g^{2}+2 g^{\prime 2} Y_{L}^{2}\right) i \not D \psi_{L}+\bar{\psi}_{R} 2 g^{\prime 2} Y_{R}^{2} i \not D \psi_{R}+2 g_{s}^{2} C_{F} \bar{q}\left(i \not D-4\left(U \mathcal{M}_{q} P_{R}+\mathcal{M}_{q}^{\dagger} U^{\dagger} P_{L}\right)\right) q\right. \\
& +\frac{V^{\prime \prime}}{v^{4}}\left(\bar{\psi}_{L} U \mathcal{M}^{\prime \prime} \psi_{R}+\text { H.c. }\right)-8 g^{\prime 2}\left(\bar{\psi}_{L} Y_{L} U \mathcal{M} Y_{R} \psi_{R}+\text { H.c. }\right) \\
& +\left(\left(3 g^{2}+g^{\prime 2}\right) \frac{v^{2}}{4} F+\frac{3}{2} \frac{F^{\prime} V^{\prime}}{F v^{2}}\right) \frac{F^{-1}}{v^{2}}\left(\bar{\psi}_{L} U\left(\frac{F^{\prime}}{2} \mathcal{M}^{\prime}-\mathcal{M}\right) \psi_{R}+\text { H.c. }\right) \\
& -\frac{8}{v^{2}} F^{-1}\left(\bar{\psi}_{L} U T^{a} \mathcal{M} \mathcal{M}^{\dagger} T^{a} \mathcal{M} \psi_{R}+\text { H.c. }\right)+\frac{2}{v^{2}}\left(\bar{\psi}_{L} U \mathcal{M}^{\prime} \mathcal{M}^{\dagger} \mathcal{M}^{\prime} \psi_{R}+\text { H.c. }\right) \\
& +\left\langle L^{\mu} L_{\mu}\right\rangle\left[\frac{F \mathcal{B}}{2 v^{2}} \bar{\psi}_{L} U \mathcal{M}^{\prime \prime} \psi_{R}-\frac{\kappa^{2}-1}{F v^{2}} \bar{\psi}_{L} U\left(\frac{F^{\prime}}{2} \mathcal{M}^{\prime}-\mathcal{M}\right) \psi_{R}+\text { H.c. }\right] \\
& +\frac{2 \kappa^{\prime}}{v^{2}} \partial^{\mu} \eta\left(i \bar{\psi}_{L} L_{\mu} U\left(F^{-1 / 2} \mathcal{M}\right)^{\prime} \psi_{R}+\text { H.c. }\right)+\frac{3 \mathcal{B}}{F v^{2}} \partial^{\mu} \eta \partial_{\mu} \eta\left(\bar{\psi}_{L} U\left(\frac{F^{\prime}}{2} \mathcal{M}^{\prime}-\mathcal{M}\right) \psi_{R}+\text { H.c. }\right) \\
& +\frac{3 F^{-2}}{2 v^{4}}\left(\bar{\psi}_{L} U\left(\frac{F^{\prime}}{2} \mathcal{M}^{\prime}-\mathcal{M}\right) \psi_{R}+\text { H.c. }\right)^{2}+\frac{1}{2 v^{4}}\left(\bar{\psi}_{L} U \mathcal{M}^{\prime \prime} \psi_{R}+\text { H.c. }\right)^{2} \\
& +\frac{4}{v^{4}}\left(i \bar{\psi}_{L} U T^{a}\left(F^{-1 / 2} \mathcal{M}\right)^{\prime} \psi_{R}+\text { H.c. }\right)^{2}+\frac{4}{v^{2}} \bar{\psi}_{L} U T^{a} \mathcal{M} F^{-1 / 2} i \not \partial\left(\mathcal{M}^{\dagger} F^{-1 / 2}\right) T^{a} U^{\dagger} \psi_{L} \\
& +\frac{4}{v^{2}} \bar{\psi}_{L} U T^{a} \mathcal{M} \mathcal{M}^{\dagger} F^{-1} T^{a} U^{\dagger} i \not D \psi_{L}+\frac{1}{v^{2}} \bar{\psi}_{L} \not L U \mathcal{M} \mathcal{M}^{\dagger} U^{\dagger} F^{-1} \psi_{L}+\frac{1}{v^{2}} \bar{\psi}_{L} U \mathcal{M}^{\prime} i \not \partial \mathcal{M}^{\dagger} U^{\dagger} \psi_{L} \\
& +\frac{1}{v^{2}} \bar{\psi}_{L} U \mathcal{M}^{\prime} \mathcal{M}^{\dagger \dagger} U^{\dagger}(i \not D+\not L) \psi_{L}-\frac{\kappa}{v^{2}} F^{-1 / 2}\left(\bar{\psi}_{L} U \mathcal{M}^{\prime} \mathcal{M}^{\dagger} U^{\dagger} \not L \psi_{L}+\text { H.c. }\right)+\frac{3}{v^{2}} \bar{\psi}_{R} \mathcal{M}^{\dagger} F^{-1 / 2} i \not D\left(\mathcal{M} F^{-1 / 2} \psi_{R}\right) \\
& +\frac{1}{v^{2}} \bar{\psi}_{R} \mathcal{M}^{\prime \dagger} i \not D\left(\mathcal{M}^{\prime} \psi_{R}\right)-\frac{F^{-1}}{v^{2}} \bar{\psi}_{R} \mathcal{M}^{\dagger} U^{\dagger} \not U U \mathcal{M} \psi_{R}-\frac{1}{v^{2}} \bar{\psi}_{R} \mathcal{M}^{\prime \dagger} U^{\dagger} \not L U \mathcal{M}^{\prime} \psi_{R} \\
& \left.+\frac{\kappa}{v^{2}} F^{-1 / 2}\left(\bar{\psi}_{R} \mathcal{M}^{\dagger} U^{\dagger} \not U \mathcal{M}^{\prime} \psi_{R}+\text { H.c. }\right)\right\} \text {. }
\end{aligned}
$$


In these expressions, one needs to separate the contributions that renormalize the lowest-order Lagrangian $\mathcal{L}_{2}$ from those which require genuine next-to-leading order counterterms. Moreover, in the latter, one may use the equations of motion from $\mathcal{L}_{2}$. In the cases of $\mathcal{L}_{\text {div }}^{(0)}$ and $\mathcal{L}_{\text {div }}^{(1)}$, this is straightforward. In the case of $\mathcal{L}_{\text {div }}^{(1 / 2)}$, some additional manipulations are required. First, one may use the identity

$$
U T^{a} \mathcal{M} \mathcal{M}^{\dagger} T^{a} U^{\dagger}=\frac{1}{2}\left\langle\mathcal{M} \mathcal{M}^{\dagger}\right\rangle-\frac{1}{4} U \mathcal{M} \mathcal{M}^{\dagger} U^{\dagger} .
$$

Next, one needs to put $\mathcal{L}_{\text {div }}^{(1 / 2)}$ into a manifestly Hermitian form. This can be done by adding total-derivative terms. Specifically, using the equations of motion at leading order

$$
i \not D \psi_{L}=U \mathcal{M} \psi_{R}, \quad i \not D \psi_{R}=\mathcal{M}^{\dagger} U^{\dagger} \psi_{L},
$$

one establishes the identities

$$
\begin{aligned}
& \frac{3}{v^{2}} \bar{\psi}_{R} \mathcal{M}^{\dagger} F^{-1 / 2} i \not D\left(\mathcal{M} F^{-1 / 2} \psi_{R}\right)=\frac{3 i}{2 v^{2}} \partial_{\mu}\left(F^{-1} \bar{\psi}_{R} \gamma^{\mu} \mathcal{M}^{\dagger} \mathcal{M} \psi_{R}\right)+\frac{3 F^{-1}}{2 v^{2}} \bar{\psi}_{R}\left(\mathcal{M}^{\dagger} i \not \supset \mathcal{M}-i \not \supset \mathcal{M}^{\dagger} \mathcal{M}\right) \psi_{R} \\
& +\frac{3 F^{-1}}{2 v^{2}}\left(\bar{\psi}_{R} \mathcal{M}^{\dagger} \mathcal{M} \mathcal{M}^{\dagger} U^{\dagger} \psi_{L}+\bar{\psi}_{L} U \mathcal{M} \mathcal{M}^{\dagger} \mathcal{M} \psi_{R}\right) \\
& \frac{1}{v^{2}} \bar{\psi}_{R} \mathcal{M}^{\prime \dagger} i \not D\left(\mathcal{M}^{\prime} \psi_{R}\right)=\frac{i}{2 v^{2}} \partial_{\mu}\left(\bar{\psi}_{R} \gamma^{\mu} \mathcal{M}^{\prime \dagger} \mathcal{M}^{\prime} \psi_{R}\right)+\frac{1}{2 v^{2}} \bar{\psi}_{R}\left(\mathcal{M}^{\prime \dagger} i \not \supset \mathcal{M}^{\prime}-i \not \supset \mathcal{M}^{\prime \dagger} \mathcal{M}^{\prime}\right) \psi_{R} \\
& +\frac{1}{2 v^{2}}\left(\bar{\psi}_{R} \mathcal{M}^{\prime \dagger} \mathcal{M}^{\prime} \mathcal{M}^{\dagger} U^{\dagger} \psi_{L}+\bar{\psi}_{L} U \mathcal{M} \mathcal{M}^{\prime \dagger} \mathcal{M}^{\prime} \psi_{R}\right), \\
& \frac{1}{v^{2}} \bar{\psi}_{L} U \mathcal{M}^{\prime}\left(i \not \partial \mathcal{M}^{\prime \dagger}\right) U^{\dagger} \psi_{L}+\frac{1}{v^{2}} \bar{\psi}_{L} U \mathcal{M}^{\prime} \mathcal{M}^{\prime \dagger} U^{\dagger}(i \not D+\not L) \psi_{L} \\
& =\frac{i}{2 v^{2}} \partial_{\mu}\left(\bar{\psi}_{L} U \mathcal{M}^{\prime} \mathcal{M}^{\dagger \dagger} U^{\dagger} \gamma^{\mu} \psi_{L}\right)+\frac{1}{2 v^{2}} \bar{\psi}_{L} U\left(\mathcal{M}^{\prime} i \not \partial \mathcal{M}^{\prime \dagger}-i \not \supset \mathcal{M}^{\prime} \mathcal{M}^{\prime \dagger}\right) U^{\dagger} \psi_{L} \\
& +\frac{1}{2 v^{2}} \bar{\psi}_{L}\left(U \mathcal{M}^{\prime} \mathcal{M}^{\prime \dagger} U^{\dagger} \not L+\not L U \mathcal{M}^{\prime} \mathcal{M}^{\prime \dagger} U^{\dagger}\right) \psi_{L}+\frac{1}{2 v^{2}}\left(\bar{\psi}_{L} U \mathcal{M}^{\prime} \mathcal{M}^{\prime \dagger} \mathcal{M} \psi_{R}+\bar{\psi}_{R} \mathcal{M}^{\dagger} \mathcal{M}^{\prime} \mathcal{M}^{\prime \dagger} U^{\dagger} \psi_{L}\right) \text {, } \\
& \frac{2 F^{-1 / 2}}{v^{2}} \bar{\psi}_{L}\left\langle\mathcal{M} i \not \partial\left(\mathcal{M}^{\dagger} F^{-1 / 2}\right)\right\rangle \psi_{L}+\frac{2 F^{-1}}{v^{2}} \bar{\psi}_{L}\left\langle\mathcal{M} \mathcal{M}^{\dagger}\right\rangle i \not D \psi_{L} \\
& =\frac{i}{v^{2}} \partial_{\mu}\left(F^{-1} \bar{\psi}_{L} \gamma^{\mu}\left\langle\mathcal{M} \mathcal{M}^{\dagger}\right\rangle \psi_{L}\right)+\frac{F^{-1}}{v^{2}} \bar{\psi}_{L}\left\langle\mathcal{M} i \not \supset \mathcal{M}^{\dagger}-i \not \supset \mathcal{M} \mathcal{M}^{\dagger}\right\rangle \psi_{L} \\
& +\frac{F^{-1}}{v^{2}} \bar{\psi}_{L} U\left\langle\mathcal{M} \mathcal{M}^{\dagger}\right\rangle \mathcal{M} \psi_{R}+\frac{F^{-1}}{v^{2}} \bar{\psi}_{R} \mathcal{M}^{\dagger}\left\langle\mathcal{M} \mathcal{M}^{\dagger}\right\rangle U^{\dagger} \psi_{L}, \\
& -\frac{F^{-1 / 2}}{v^{2}} \bar{\psi}_{L} U \mathcal{M} i \not \partial\left(\mathcal{M}^{\dagger} F^{-1 / 2}\right) U^{\dagger} \psi_{L}-\frac{F^{-1}}{v^{2}} \bar{\psi}_{L} U \mathcal{M} \mathcal{M}^{\dagger} U^{\dagger} i \not D \psi_{L}+\frac{F^{-1}}{v^{2}} \bar{\psi}_{L} \not L U \mathcal{M} \mathcal{M}^{\dagger} U^{\dagger} \psi_{L} \\
& =-\frac{i}{2 v^{2}} \partial_{\mu}\left(F^{-1} \bar{\psi}_{L} \gamma^{\mu} U \mathcal{M} \mathcal{M}^{\dagger} U^{\dagger} \psi_{L}\right)-\frac{F^{-1}}{2 v^{2}} \bar{\psi}_{L} U\left(\mathcal{M} i \not \supset \mathcal{M}^{\dagger}-i \not \supset \mathcal{M} \mathcal{M}^{\dagger}\right) U^{\dagger} \psi_{L} \\
& +\frac{F^{-1}}{2 v^{2}} \bar{\psi}_{L}\left(\not L U \mathcal{M} \mathcal{M}^{\dagger} U^{\dagger}+U \mathcal{M} \mathcal{M}^{\dagger} U^{\dagger} \not L\right) \psi_{L}-\frac{F^{-1}}{2 v^{2}} \bar{\psi}_{L} U \mathcal{M} \mathcal{M}^{\dagger} \mathcal{M} \psi_{R}-\frac{F^{-1}}{2 v^{2}} \bar{\psi}_{R} \mathcal{M}^{\dagger} \mathcal{M} \mathcal{M}^{\dagger} U^{\dagger} \psi_{L} \text {, }
\end{aligned}
$$

so that, upon dropping the total derivatives, $\mathcal{L}_{\text {div }}^{(1 / 2)}$ may be rewritten as 


$$
\begin{aligned}
& \mathcal{L}_{\mathrm{div}}^{(1 / 2)}=-\frac{1}{16 \pi^{2}} \frac{1}{d-4}\left\{\bar{\psi}_{L}\left(\frac{3}{2} g^{2}+2 g^{\prime 2} Y_{L}^{2}\right) i \not D \psi_{L}+\bar{\psi}_{R} 2 g^{\prime 2} Y_{R}^{2} i \not D \psi_{R}\right. \\
& +2 g_{s}^{2} C_{F} \bar{q}\left(i \not D-4\left(U \mathcal{M}_{q} P_{R}+\mathcal{M}_{q}^{\dagger} U^{\dagger} P_{L}\right)\right) q \\
& +\frac{V^{\prime \prime}}{v^{4}}\left(\bar{\psi}_{L} U \mathcal{M}^{\prime \prime} \psi_{R}+\text { H.c. }\right)-8 g^{2}\left(\bar{\psi}_{L} Y_{L} U \mathcal{M} Y_{R} \psi_{R}+\text { H.c. }\right) \\
& +\left(\left(3 g^{2}+g^{\prime 2}\right) \frac{v^{2}}{4} F+\frac{3}{2} \frac{F^{\prime} V^{\prime}}{F v^{2}}\right) \frac{F^{-1}}{v^{2}}\left(\bar{\psi}_{L} U\left(\frac{F^{\prime}}{2} \mathcal{M}^{\prime}-\mathcal{M}\right) \psi_{R}+\text { H.c. }\right) \\
& +\frac{3}{v^{2}} F^{-1}\left(\bar{\psi}_{L} U \mathcal{M} \mathcal{M}^{\dagger} \mathcal{M} \psi_{R}+\text { H.c. }\right)-\frac{3}{v^{2}} F^{-1}\left(\bar{\psi}_{L} U\left\langle\mathcal{M} \mathcal{M}^{\dagger}\right\rangle \mathcal{M} \psi_{R}+\text { H.c. }\right) \\
& +\frac{2}{v^{2}}\left(\bar{\psi}_{L} U \mathcal{M}^{\prime} \mathcal{M}^{\dagger} \mathcal{M}^{\prime} \psi_{R}+\text { H.c. }\right)+\frac{1}{2 v^{2}}\left(\bar{\psi}_{L} U \mathcal{M} \mathcal{M}^{\prime \dagger} \mathcal{M}^{\prime} \psi_{R}+\text { H.c. }\right)+\frac{1}{2 v^{2}}\left(\bar{\psi}_{L} U \mathcal{M}^{\prime} \mathcal{M}^{\prime \dagger} \mathcal{M} \psi_{R}+\text { H.c. }\right) \\
& +\frac{3 F^{-1}}{2 v^{2}} \bar{\psi}_{R}\left(\mathcal{M}^{\dagger} i \not \partial \mathcal{M}-i \not \partial \mathcal{M}^{\dagger} \mathcal{M}\right) \psi_{R}+\frac{1}{2 v^{2}} \bar{\psi}_{R}\left(\mathcal{M}^{\dagger \dagger} i \not \partial \mathcal{M}^{\prime}-i \not \partial \mathcal{M}^{\prime \dagger} \mathcal{M}^{\prime}\right) \psi_{R} \\
& -\frac{F^{-1}}{2 v^{2}} \bar{\psi}_{L} U\left(\mathcal{M} i \not \partial \mathcal{M}^{\dagger}-i \not \partial \mathcal{M} \mathcal{M}^{\dagger}\right) U^{\dagger} \psi_{L}+\frac{1}{2 v^{2}} \bar{\psi}_{L} U\left(\mathcal{M}^{\prime} i \not \partial \mathcal{M}^{\prime \dagger}-i \not \partial \mathcal{M}^{\prime} \mathcal{M}^{\prime \dagger}\right) U^{\dagger} \psi_{L} \\
& +\frac{F^{-1}}{v^{2}} \bar{\psi}_{L}\left\langle\mathcal{M} i \not \partial \mathcal{M}^{\dagger}-i \not \partial \mathcal{M} \mathcal{M}^{\dagger}\right\rangle \psi_{L}-\frac{F^{-1}}{v^{2}} \bar{\psi}_{R} \mathcal{M}^{\dagger} U^{\dagger} \not U \mathcal{M} \psi_{R}-\frac{1}{v^{2}} \bar{\psi}_{R} \mathcal{M}^{\prime \dagger} U^{\dagger} \mathcal{L} U^{\prime} \psi_{R} \\
& +\frac{\kappa}{v^{2}} F^{-1 / 2}\left(\bar{\psi}_{R} \mathcal{M}^{\dagger} U^{\dagger} \not U \mathcal{M}^{\prime} \psi_{R}+\text { H.c. }\right)-\frac{\kappa}{v^{2}} F^{-1 / 2}\left(\bar{\psi}_{L} U \mathcal{M}^{\prime} \mathcal{M}^{\dagger} U^{\dagger} \not \psi_{L}+\text { H.c. }\right) \\
& +\frac{1}{2 v^{2}} \bar{\psi}_{L}\left(U \mathcal{M}^{\prime} \mathcal{M}^{\prime \dagger} U^{\dagger} \not L+\not L U \mathcal{M}^{\prime} \mathcal{M}^{\prime \dagger} U^{\dagger}\right) \psi_{L}+\frac{F^{-1}}{2 v^{2}} \bar{\psi}_{L}\left(\not Z U \mathcal{M} \mathcal{M}^{\dagger} U^{\dagger}+U \mathcal{M M}^{\dagger} U^{\dagger} \not{L}\right) \psi_{L} \\
& +\left\langle L^{\mu} L_{\mu}\right\rangle\left[\frac{F \mathcal{B}}{2 v^{2}} \bar{\psi}_{L} U \mathcal{M}^{\prime \prime} \psi_{R}-\frac{\kappa^{2}-1}{F v^{2}} \bar{\psi}_{L} U\left(\frac{F^{\prime}}{2} \mathcal{M}^{\prime}-\mathcal{M}\right) \psi_{R}+\text { H.c. }\right]+\frac{2 \kappa^{\prime}}{v^{2}} \partial^{\mu} \eta\left(i \bar{\psi}_{L} L_{\mu} U\left(F^{-1 / 2} \mathcal{M}\right)^{\prime} \psi_{R}+\text { H.c. }\right) \\
& +\frac{3 \mathcal{B}}{F v^{2}} \partial^{\mu} \eta \partial_{\mu} \eta\left(\bar{\psi}_{L} U\left(\frac{F^{\prime}}{2} \mathcal{M}^{\prime}-\mathcal{M}\right) \psi_{R}+\text { H.c. }\right)+\frac{3 F^{-2}}{2 v^{4}}\left(\bar{\psi}_{L} U\left(\frac{F^{\prime}}{2} \mathcal{M}^{\prime}-\mathcal{M}\right) \psi_{R}+\text { H.c. }\right)^{2} \\
& \left.+\frac{1}{2 v^{4}}\left(\bar{\psi}_{L} U \mathcal{M}^{\prime \prime} \psi_{R}+\text { H.c. }\right)^{2}+\frac{4}{v^{4}}\left(i \bar{\psi}_{L} U T^{a}\left(F^{-1 / 2} \mathcal{M}\right)^{\prime} \psi_{R}+\text { H.c. }\right)^{2}\right\} \text {. }
\end{aligned}
$$

[1] A. David et al. (LHC Higgs Cross Section Working Group), Report No. LHCHXSWG-2012-001, https://inspirehep.net/ literature/1184164

[2] G. Buchalla, O. Catà, A. Celis, and C. Krause, Phys. Lett. B 750, 298 (2015).

[3] J. de Blas, O. Eberhardt, and C. Krause, J. High Energy Phys. 07 (2018) 048.

[4] F. Feruglio, Int. J. Mod. Phys. A 08, 4937 (1993).

[5] J. Bagger, V. Barger, K. Cheung, J. Gunion, T. Han, G. A. Ladinsky, R. Rosenfeld, and C. -P. Yuan, Phys. Rev. D 49, 1246 (1994).

[6] V. Koulovassilopoulos and R. S. Chivukula, Phys. Rev. D 50, 3218 (1994).

[7] C. P. Burgess, J. Matias, and M. Pospelov, Int. J. Mod. Phys. A 17, 1841 (2002).

[8] L. M. Wang and Q. Wang, arXiv:hep-ph/0605104.

[9] B. Grinstein and M. Trott, Phys. Rev. D 76, 073002 (2007).
[10] R. Contino, C. Grojean, M. Moretti, F. Piccinini, and R. Rattazzi, J. High Energy Phys. 05 (2010) 089.

[11] R. Contino, arXiv:1005.4269.

[12] R. Alonso, M. B. Gavela, L. Merlo, S. Rigolin, and J. Yepes, Phys. Lett. B 722, 330 (2013); 726, 926(E) (2013).

[13] R. Alonso, M. B. Gavela, L. Merlo, S. Rigolin, and J. Yepes, Phys. Rev. D 87, 055019 (2013).

[14] G. Buchalla, O. Catà, and C. Krause, Nucl. Phys. B880, 552 (2014); B913, 475(E) (2016).

[15] G. Buchalla, O. Catà, and C. Krause, Phys. Lett. B 731, 80 (2014).

[16] G. Buchalla, O. Catà, A. Celis, M. Knecht, and C. Krause, Nucl. Phys. B928, 93 (2018).

[17] R. Alonso, K. Kanshin, and S. Saa, Phys. Rev. D 97, 035010 (2018).

[18] R. Urech, Nucl. Phys. B433, 234 (1995).

[19] M. Knecht, H. Neufeld, H. Rupertsberger, and P. Talavera, Eur. Phys. J. C 12, 469 (2000). 
[20] J. Gasser and H. Leutwyler, Ann. Phys. (N.Y.) 158, 142 (1984).

[21] H. Politzer, Nucl. Phys. B172, 349 (1980).

[22] C. Arzt, Phys. Lett. B 342, 189 (1995).

[23] A. Denner, G. Weiglein, and S. Dittmaier, Nucl. Phys. B440, 95 (1995).

[24] G. Buchalla and O. Catà, J. High Energy Phys. 07 (2012) 101.

[25] W. Buchmüller and D. Wyler, Nucl. Phys. B268, 621 (1986).

[26] B. Grzadkowski, M. Iskrzynski, M. Misiak, and J. Rosiek, J. High Energy Phys. 10 (2010) 085.

[27] G. Buchalla, O. Catà, and C. Krause, Nucl. Phys. B894, 602 (2015).

[28] E. E. Jenkins, A. V. Manohar, and M. Trott, J. High Energy Phys. 10 (2013) 087.

[29] E. E. Jenkins, A. V. Manohar, and M. Trott, J. High Energy Phys. 01 (2014) 035.

[30] R. Alonso, E. E. Jenkins, A. V. Manohar, and M. Trott, J. High Energy Phys. 04 (2014) 159.

[31] R. Alonso, H. M. Chang, E. E. Jenkins, A. V. Manohar, and B. Shotwell, Phys. Lett. B 734, 302 (2014).

[32] G. Buchalla, A. Celis, C. Krause, and J. N. Toelstede, arXiv: 1904.07840.

[33] A. Celis, J. Fuentes-Martin, A. Vicente, and J. Virto, Eur. Phys. J. C 77, 405 (2017).

[34] R. L. Delgado, A. Dobado, and F. J. Llanes-Estrada, J. High Energy Phys. 02 (2014) 121.

[35] R. L. Delgado, A. Dobado, M. J. Herrero, and J. J. SanzCillero, J. High Energy Phys. 07 (2014) 149.
[36] M. B. Gavela, K. Kanshin, P. A. N. Machado, and S. Saa, J. High Energy Phys. 03 (2015) 043.

[37] R. L. Delgado, A. Dobado, and F. J. Llanes-Estrada, Phys. Rev. D 91, 075017 (2015).

[38] F. K. Guo, P. Ruiz-Femenía, and J. J. Sanz-Cillero, Phys. Rev. D 92, 074005 (2015).

[39] R. Alonso, E. E. Jenkins, and A. V. Manohar, Phys. Lett. B 754, 335 (2016).

[40] T. Appelquist and C. W. Bernard, Phys. Rev. D 22, 200 (1980).

[41] A. C. Longhitano, Phys. Rev. D 22, 1166 (1980).

[42] A. C. Longhitano, Nucl. Phys. B188, 118 (1981).

[43] M. J. Herrero and E. Ruiz Morales, Nucl. Phys. B418, 431 (1994).

[44] M. J. Herrero and E. Ruiz Morales, Nucl. Phys. B437, 319 (1995).

[45] S. Dittmaier and C. Grosse-Knetter, Nucl. Phys. B459, 497 (1996).

[46] R. Mertig, M. Böhm, and A. Denner, Comput. Phys. Commun. 64, 345 (1991).

[47] V. Shtabovenko, R. Mertig, and F. Orellana, Comput. Phys. Commun. 207, 432 (2016).

[48] Wolfram Research and Inc., Mathematica, Version 11.1 (2017).

[49] V. I. Borodulin, R. N. Rogalyov, and S. R. Slabospitskii, arXiv: 1702.08246.

[50] C. Krause, A. Pich, I. Rosell, J. Santos, and J. J. SanzCillero, J. High Energy Phys. 05 (2019) 092. 